\title{
The membrane IgE tail imparts unique signaling properties to the $B$ cell antigen receptor
}

\author{
Dissertation \\ for the award of the degree \\ "Doctor rerum naturalium" \\ of the Georg-August-Universität Göttingen
}

within the doctoral program "Molecular Biology"

of the Georg-August University School of Science (GAUSS)

\author{
submitted by \\ Kanika Vanshylla \\ from \\ New Delhi, India
}

Göttingen 2016 


\section{Thesis Committee}

Prof. Dr. Jürgen Wienands, Institute for Cellular and Molecular Immunology, University Medical Center, Göttingen

Prof. Dr. Matthias Dobbelstein, Department of Molecular Oncology, University Medical Center, Göttingen

Dr. Roland Dosch, Department of Developmental Biochemistry, University Medical Center, Göttingen

Dr. Niklas Engels, Institute for Cellular and Molecular Immunology, University Medical Center, Göttingen

\section{Members of the Examination Board}

Referee: Prof. Dr. Jürgen Wienands, Institute for Cellular and Molecular Immunology, University Medical Center, Göttingen

$2^{\text {nd }}$ Referee: Prof. Dr. Matthias Dobbelstein, Department of Molecular Oncology, University Medical Center, Göttingen

\section{Further members of the Examination Board}

Dr. Roland Dosch, Department of Developmental Biochemistry, University Medical Center, Göttingen

Prof. Dr. Steven Johnsen, Translational Cancer Research, University Medical Center, Göttingen

Prof Dr. Peter Rehling, Department of Cellular Biochemistry, University Medical Center, Göttingen

Prof. Dr. Lutz Walter, Department of Primate Genetics, German Primate Center, Göttingen

Date of oral examination: December $12^{\text {th }} 2016$

This doctoral thesis was conducted in the Institute for Cellular and Molecular Immunology at the Georg-August University in Göttingen, Germany from May 2013 to October 2016 under the supervision of Dr. Niklas Engels and Prof. Dr. Jürgen Wienands. 


\section{Table of Contents}

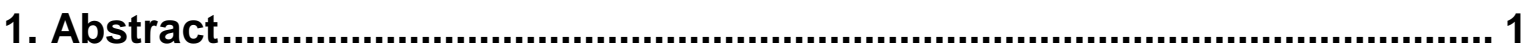

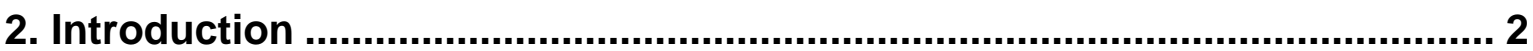

2.1 The immune system of vertebrates ....................................................... 2

2.1.1 The innate immune system - First line of defense against pathogens............... 2

2.1.2 Adaptive immunity - the second arm of the immune system ……………….... 2

2.2 Antibody mediated humoral immunity ……….................................................. 3

2.3 Antigen-induced canonical BCR signaling pathways .................................... 4

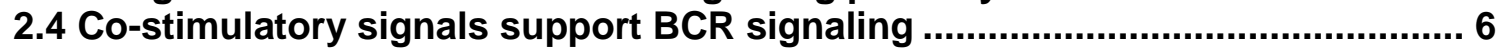

2.5 Germinal centers - sites for formation of B cell memory ................................. 7

2.6 The biological role of $\operatorname{lgE}$......................................................................... 8

2.7 Is there a true IgE memory compartment? .................................................... 9

2.8 How B cells remember - cellular features of memory $B$ cell responses...........10

2.9 BCR signal amplification via the immunoglobulin tail tyrosine (ITT) motif .....10

2.10 Additional molecular features of the mlgG tail ...........................................12

2.11 How does the ITT influence mlgE-BCR signaling? .......................................13

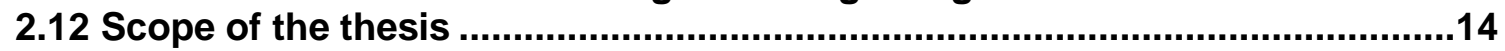

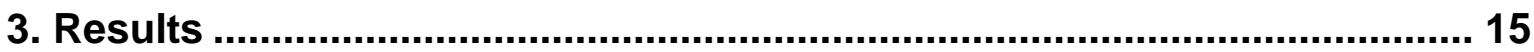

3.1 The ITT increases the sensitivity of mlgE-BCRs by utilizing the adaptor proteins Grb2 and Grap............................................................................15

3.2 Deficiency of Grb2 and Grap prevents optimal activation of the canonical

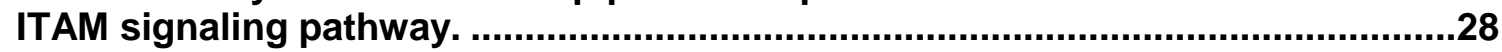

3.3 Human $\mathrm{mlgE}$ can function independently of the $\lg \alpha / \lg \beta$ heterodimer...............33

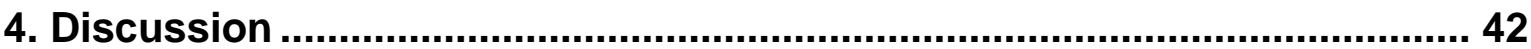

4.1 BCR signal amplification by the ITT signaling module in the context of mlgEBCRs.

4.2 The complex role of adaptor proteins in BCR signaling - regulation of ITAM signaling by Grb2 and Grap.

4.3 It all comes down to the tail - human mlgE expression and ITT signaling in the

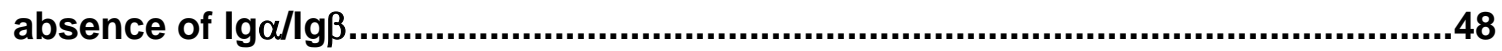

4.4 Conclusions and perspectives........................................................................51

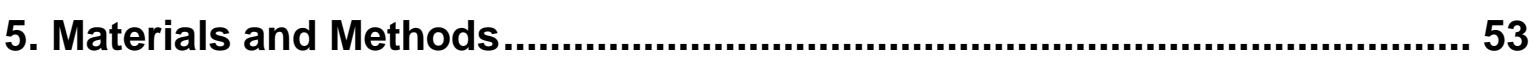

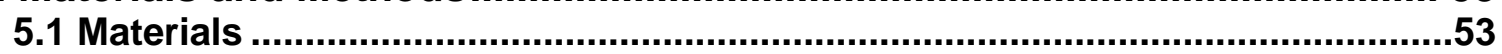

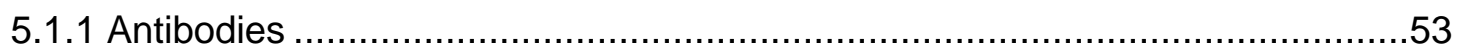

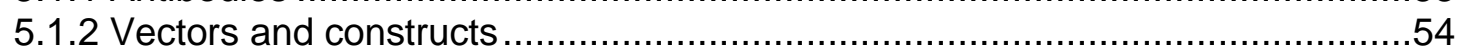

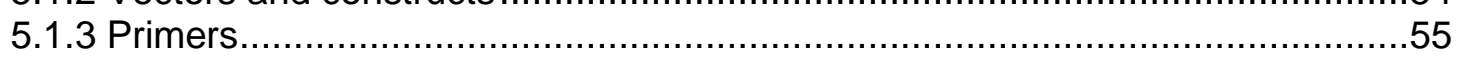

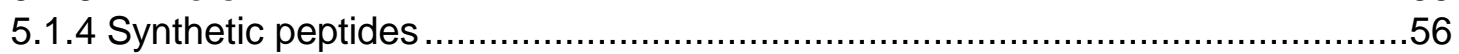

5.1.5 Mammalian cell lines .........................................................................56

5.1.6 Cell culture media for mammalian cell lines...............................................57

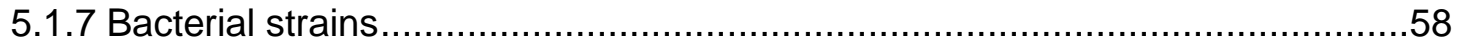

5.1.8 Media and plates for bacteria ..............................................................58

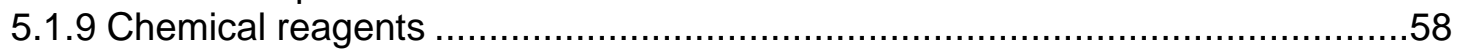

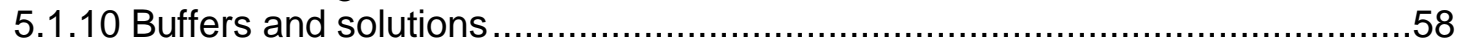

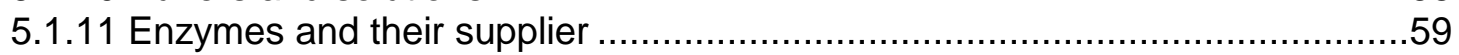

5.1.12 Additional materials and their supplier ..................................................6

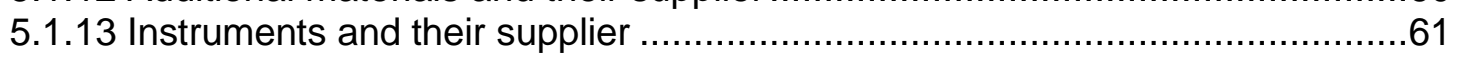

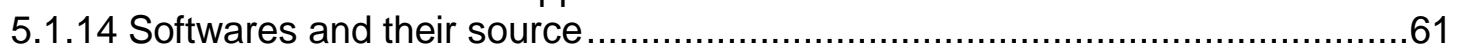


5.2 Methods .61

5.2.1 Culturing mammalian cells

5.2.2 Freezing and thawing of mammalian cells ................................................61

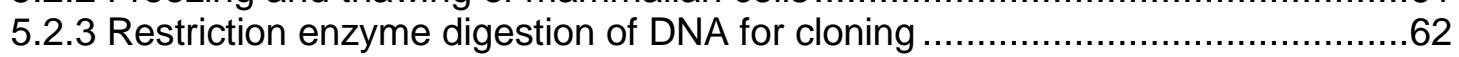

5.2.4 Agarose gel electrophoresis and purification of DNA fragments .....................62

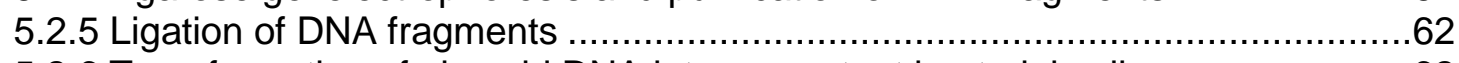

5.2.6 Transformation of plasmid DNA into competent bacterial cells ........................62

5.2.7 Mini- and midi-preparations of plasmid DNA .............................................63

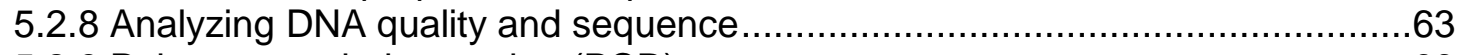

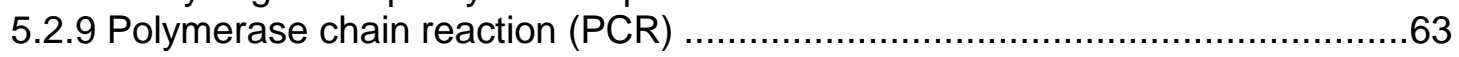

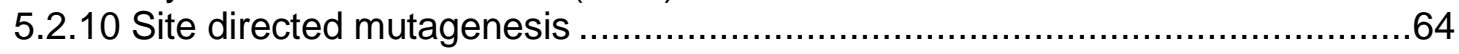

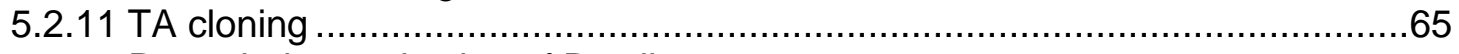

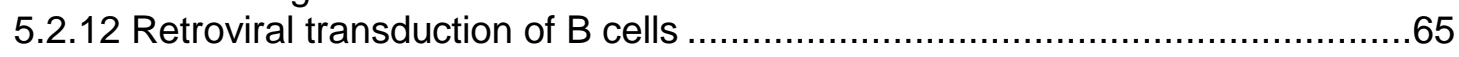

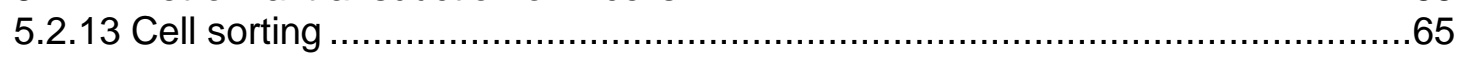

5.2.14 Surface staining and protein expression analysis via flow cytometry .............66

5.2.15 Preparation of B cell lysates .............................................................66

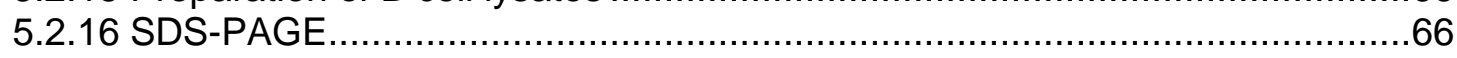

5.2.17 Immunoblotting / western blotting ......................................................67

5.2.18 Affinity purification using antibodies or biotinylated peptides .......................67

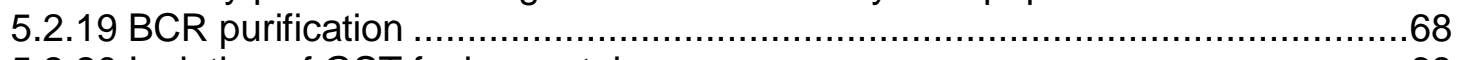

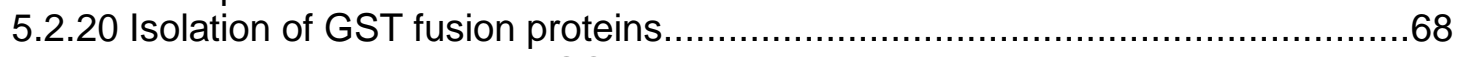

5.2.21 Affinity purification using GST fusion proteins..........................................69

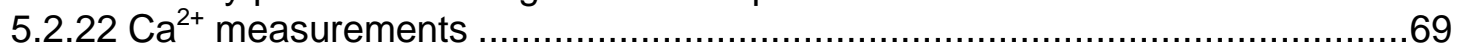

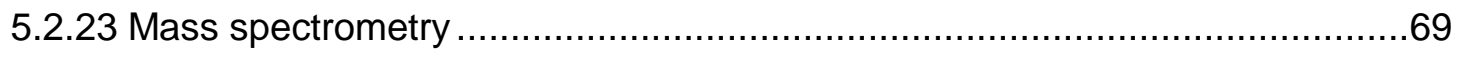

6. References

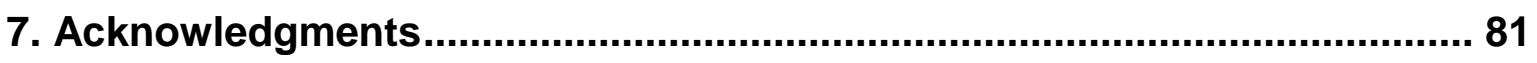

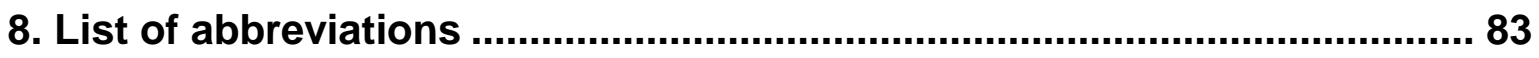

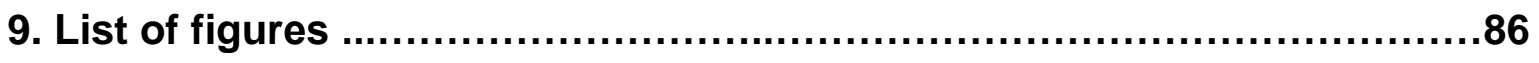

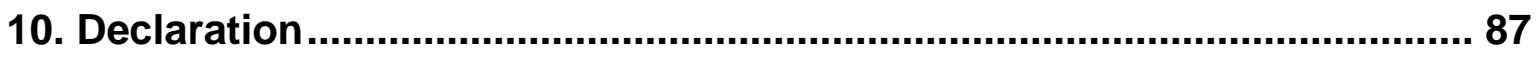

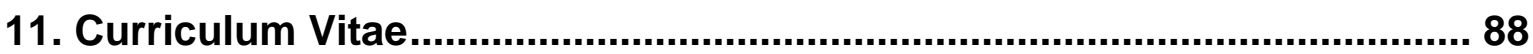




\section{Abstract}

Robust memory antibody responses are a distinguishing feature of humoral immunity and are typically marked by elevated serum immunoglobulin (Ig) titers that rapidly and effectively neutralize the invading pathogen. However, relatively little is known about the molecular mechanisms that govern the activation of memory B cells. In particular, the biology of IgE-expressing memory $B$ cells remains elusive due to the scarcity of this $B$ cell population, despite the clinical importance of $\lg E$ as a major driver for immediate hypersensitivity reactions. By analyzing human membrane bound $\lg \mathrm{E}(\mathrm{m} \lg \mathrm{E})$ in $\mathrm{B}$ cell lines, this work focused on the molecular composition and signaling features of mlgEcontaining $B$ cell antigen receptors (BCRs).

A previously described mlg-intrinsic motif called the immunoglobulin tail tyrosine (ITT), which is exclusively present in the cytoplasmic tails of $\mathrm{mlg} G$ and $\mathrm{mlg} E$, is known to augment IgG1 responses in a mouse model system. The mlgG-ITT amplifies BCR signaling via a Grb2-Btk complex that enhances intracellular $\mathrm{Ca}^{2+}$ mobilization. However, I found that unlike the mlgG-ITT which exclusively signals via the adaptor protein Grb2, the mlgE-ITT also employs the Grb2 related adaptor protein (Grap), which together with Grb2, plays an important role in mlgE-BCR signaling. The absence of these adaptor proteins severely impairs the capability of mlgE-BCRs to mobilize $\mathrm{Ca}^{2+}$ and activate the mitogen activated protein (MAP) kinase extracellular signal regulated kinase (ERK) pathway, both of which are central pathways for B cell activation, proliferation and differentiation. Therefore, by enhancing BCR signaling via the ITT-Grb2/Grap module, mlgE-BCRs lower the activation threshold of mlgE-expressing $B$ cells.

Furthermore, a new role for Grb2 and Grap in the canonical immunoreceptor tyrosinebased activation motif (ITAM) signaling pathway of ITT-less BCRs was revealed, since mlgM-BCRs also require these proteins for optimal $\mathrm{Ca}^{2+}$ signaling. Grb2 and Grap contribute to ITAM-induced $\mathrm{Ca}^{2+}$ mobilization through improved activation of the $\mathrm{Ca}^{2+}$ initiation complex proteins Syk, SLP65 and PLC $\gamma 2$. Therefore, Grb2 and Grap are not only necessary for mlgE- but also mlgM-BCR signaling in human B cells.

Intriguingly, unlike the mlgM molecule, the human mlgE molecule can be expressed on the surface of $B$ cells in the absence of the $\lg \alpha / \lg \beta$ heterodimer, a characteristic that depends on the cytoplasmic tail of mlgE. The mlgE-ITT motif is signaling competent in the absence of $\lg \alpha / \lg \beta$ and requires the catalytic activity of the tyrosine kinase Syk. These results highlight differences in signaling mechanisms employed by different BCR isotypes. While mlgM-expressing $B$ cells are restricted by the $\lg \alpha / \lg \beta$ heterodimer for $B C R$ expression and signaling, mlgE-expressing B cells can utilize the ITT motif independently of $\lg \alpha / \lg \beta$ for activation of signaling pathways. This non-canonical mlgE signaling via the ITT motif provides preliminary insight into novel signaling mechanisms that could be regulating the fate of the $\mathrm{IgE}$ memory and plasma $\mathrm{B}$ cell compartment. 


\section{Introduction}

\subsection{The immune system of vertebrates}

\subsubsection{The innate immune system - First line of defense against pathogens}

The innate arm of the immune system coordinates the early phase of an immune response to pathogens. For instance, the epithelial layer not only physically impedes the entry of pathogens but also actively secretes antimicrobial peptides like defensins (Selsted and Ouelette 2005). Pathogens that overcome these barriers are ingested and degraded by phagocytic cells like neutrophils or macrophages, in a process called phagocytosis (Segal 2005). Larger pathogens like helminths cannot be phagocytosed and are eliminated by the combined action of mast cells, basophils and eosinophils that secrete cytotoxic granules (Allen and Maizels 2011). The circulating complement proteins mediate killing of pathogens via formation of membrane attack complexes that lyse membranes (Zipfel and Skerka 2009).

The innate immune cells do not always work alone, but also in concert with the adaptive immune cells, B and T lymphocytes, which express receptors that bind specifically to molecules called antigens (Iwasaki and Medzhitov 2015). Production of inflammatory cytokines by macrophages and antigen presentation by dendritic cells contribute to lymphocyte recruitment and activation. Conversely, antibodies work with the innate immune system by activating the complement system or by stimulating mast cells to secrete granules via immunoglobulin $\mathrm{E}$ ( $\mathrm{IgE})$ - $F c$ epsilon receptor ( $F c \varepsilon R$ ) interactions. Natural killer cells function at the junction of innate and adaptive immunity since they can directly recognize and kill virus-infected cells, but are also involved in processes like macrophage activation and antibody-dependent cell-mediated cytotoxicity (ADCC) (Vivier et al 2008). Though the innate immune system is fast in its response and peaks within hours of infection, it lacks the capability to precisely recognize and remember its encounter with the antigen. In contrast, the adaptive immune system can target specific antigens and provide long term immunity (Janeway and Medzitov 2002).

\subsubsection{Adaptive immunity - the second arm of the immune system}

The adaptive immune system comprises $B$ and $T$ lymphocytes that in mammals are generated in the bone marrow and thymus, respectively. Upon maturation, these lymphocytes can either be found in secondary lymphoid organs like the spleen and lymph nodes, or in the blood and lymph where they scan the body for antigens. Recognition of the cognate antigen by these cells leads to expansion of antigen specific clones. These cells then effectively clear the antigen via the action of antibodies, which are secreted by differentiated $B$ cells called plasma cells, or via direct cell killing by cytotoxic $T$ cells (Cooper 2015, Zuniga-Pfluecker 2004). 


\subsection{Antibody mediated humoral immunity}

Antibodies are key weapons of the humoral immune system and are responsible for neutralizing and eliminating extracellular microbes. One of the first recorded instances of knowledge about humoral immunity dates back to China in the 1500s where smallpox inoculations were used to boost the immune system (now called vaccination). It was in the late 1800s that antibodies were identified as the principle factor in the serum that targets pathogens (Von Behring and Kitasato, 1890). Since then, a lot of progress has been made in the structural and functional elucidation of the antibody molecule, culminating in the awarding of many Nobel prizes in the field. Today, besides the use of antibodies in research applications, targeted monoclonal antibody therapy is being used to treat many autoimmune disorders, allergies and cancers. The latest development in this regard is the use of chimeric antigen receptors (CARs) as therapy to treat B cell malignancies (Maude et al 2014). Thus, understanding the biology of B cell-mediated antibody responses can have many applications in vaccine development, cancer therapy and treatment of autoimmune diseases and allergies.

At a functional level, secreted antibodies can initiate the elimination of the pathogen by neutralization, FcR-induced phagocytosis, ADCC or complement-mediated lysis. Secreted antibodies are produced by plasma cells, which are the terminally differentiated 'effector' $B$ cell that are generated in a multistep process after initial recognition of the antigen by naïve or memory B cells. First contact with an antigen induces naïve B cells to mount a primary or naïve response, which is mainly dominated by the production of IgM-type antibodies (Yuan et al 1977). This initial burst of $\operatorname{lgM}$ antibodies is important for containment of the pathogen. Besides undergoing differentiation into lgM-secreting plasma cells, some naïve B cells form memory cells (Gray 1993). A fraction of memory B cells switch their antibody isotype to $\lg G, \lg E$ or $\lg A$ and produce higher affinity antibodies (Honjo et al 2002, Mc-Heyzer-Williams et al 1991). Thus, re-challenge by the same pathogen results in a secondary or memory response, which is marked by the presence of large quantities of high affinity, mainly lgG antibodies in the serum that rapidly mediate the elimination of the pathogen (Yuan et al 1977, Hangartner et al 2006, Dogan et al 2009, Pape et al 2011).

In order to start the process of B cell activation and differentiation, the pathogen needs to be specifically recognized by the cells. This is done by the membrane-bound immunoglobulin ( $\mathrm{mlg}$ ) molecule that is part of the B cell antigen receptor (BCR) complex (Reth 1992). The antigen binding region of the mlg is generated by somatic recombination of gene segments that encode for the mlg during $B$ cell development in the bone marrow (Brack et al 1978). This process of random gene re-arrangement is called V(D)J recombination and is responsible for the wide diversity of antigens that can be detected by $B$ cells. Binding of antigen to the BCR induces intracellular signaling cascades in the cell, which can lead to clonal expansion and differentiation. 
There are two main models that explain how binding of the antigen can initiate the activation of downstream signaling pathways. The traditional model hypothesizes that binding of multivalent antigens induces crosslinking of many BCRs which are monomeric in the resting state. The oligomerization of the BCRs allows the activation of intracellular kinases, thereby kicking off BCR signaling (Metzger 1992, Tolar et al 2009). However, in the course of time, it was revealed that monovalent antigens can also induce intracellular BCR signaling (Kim et al 2006, Volkmann et al 2016). This gave rise to the so-called dissociation activation model, which proposes that in the resting state, $\mathrm{mlgM}$ - and mlgDBCRs are present in pre-formed oligomers in the plasma membrane. The binding of antigen displaces these oligomers, causing kinase induced opening of the receptor which culminates in intracellular BCR signaling (Schamel and Reth 2000, Yang and Reth 2010, Klaesner et al 2014). Regardless of these modes of activation of the BCR by antigen, the main intracellular signaling pathways following BCR stimulation remain the same and are discussed next.

\subsection{Antigen-induced canonical BCR signaling pathways}

Naïve B cells express either mlgM- and/or mlgD-BCRs on their cell surface (Havran et al 1984). The mlgM-BCR complex comprises a mlgM molecule, associated in a 1:1 stoichiometry with a heterodimer of two transmembrane proteins called $\lg \alpha$ and $\lg \beta$ (Schamel and Reth 2000). This mlgM- $\lg \alpha-\lg \beta$ complex is assembled in the endoplasmic reticulum (ER) and transported to the plasma membrane only when all components are present and correctly assembled (Venkitaraman et al 1991, Matsuuchi et al 1992). Since the mlgM (and mlgD) cytoplasmic tails only contain three amino acids without any known signaling capabilities, antigen-induced BCR signaling begins with the phosphorylation of immunoreceptor tyrosine-based activation motifs (ITAMs) in the cytoplasmic domains of $\lg \alpha$ and $\lg \beta$ (Kim et al 1993, Campbell et al 1995, Wienands et al 1996). The ITAM has a consensus amino acid sequence of $\mathrm{D} / \mathrm{ExxD} / \mathrm{Ex}_{7} \mathrm{Yxxl} / \mathrm{Lx} \mathrm{x}_{7} \mathrm{Yxxl} / \mathrm{L}$ ( $\mathrm{x}$ being any amino acid) (Reth 1989). Binding of antigen to the mlg induces phosphorylation of the ITAM tyrosines by Src family tyrosine kinases like Lyn, Fyn or Blk (Tamir and Cambier 1998, Rolli et al 2002). The two phosphorylated ITAM tyrosine residues form a perfect binding site for the tandem Src homology 2 (SH2) domains of spleen tyrosine kinase (Syk) (Johnsen et al 1995, Kurosaki et al 1995). Upon binding to phosphorylated ITAMs, Syk phosphorylates a non-ITAM tyrosine in the C-terminus of the $\lg \alpha$ molecule. This acts as a binding site for the $\mathrm{SH} 2$ domain of the central $\mathrm{B}$ cell adaptor protein $\mathrm{SH} 2$ domain-containing leukocyte protein of $65 \mathrm{kDa}$ (SLP65), which in turn gets phosphorylated by Syk at multiple sites (Engels et al 2001, Wienands et al 1998, Zhang et al 1998). Phosphorylated SLP65 serves as a docking point for Bruton tyrosine kinase (Btk) and its substrate phosphoinositide phospholipase C-gamma-2 (PLC $\gamma 2$ ) (Kurosaki and Tsukada 2000). Together, these proteins constitute the $\mathrm{BCR} \mathrm{Ca}^{2+}$ initiation complex. The activity of most of these proteins can be monitored by testing for tyrosine phosphorylation, a modification which in most cases activates the proteins. 
Active PLC $\gamma 2$ catalyzes the breakdown of membrane phosphatidylinositol-4,5bisphosphate $\left(\mathrm{PIP}_{2}\right)$ into membrane-bound diacylglycerol (DAG) and soluble inositol1,4,5-trisphosphate $\left(\mathrm{IP}_{3}\right)$. Binding of $\mathrm{IP}_{3}$ to a $\mathrm{Ca}^{2+}$ channel called $I \mathrm{P}_{3}$ receptor $\left(\mathrm{IP}_{3} \mathrm{R}\right)$ on the ER membrane allows $\mathrm{Ca}^{2+}$ release into the cytosol. Depletion of $\mathrm{Ca}^{2+}$ in the $\mathrm{ER}$ is sensed by an ER membrane protein, stromal interaction molecule 1 (STIM1). STIM1 can directly induce $\mathrm{Ca}^{2+}$ entry from the extracellular space via opening of $\mathrm{Ca}^{2+}$ release activating channels (CRAC) channels in the plasma membrane leading to a second wave of cytosolic $\mathrm{Ca}^{2+}$ mobilization (Baba et al 2014, Engelke et al 2007). Intracellular $\mathrm{Ca}^{2+}$ levels can rapidly increase from $0.1 \mu \mathrm{M}$ to about $1 \mu \mathrm{M}$ following BCR engagement, which makes it one of the first measurable readouts of $\mathrm{B}$ cell activation. $\mathrm{Ca}^{2+}$-bound Calmodulin activates the phosphatase Calcineurin, which then dephosphorylates the transcription factor Nuclear factor of activated T cells (NFAT). Despites its name NFAT is also found in many cell types including B cells. Dephosphorylated NFAT translocates to the nucleus and induces transcriptional programs that can lead to $B$ cell activation and differentiation (Mueller and Rao 2010, Winslow et al 2006, Bhattacharyya et al 2011).

Another crucial signaling pathway activated downstream of the BCR is the extracellular signal regulated kinase (ERK)-mitogen activated protein kinase (MAPK) pathway. The adaptor protein Grb2 binds the guanine nucleotide exchange factor (GEF) Son of sevenless (Sos), which mediates the activation of the small G-protein Ras by exchanging guanosine diphosphate (GDP) with guanosine triphosphate (GTP). Ras-GTP activates a cascade of enzymes, namely Raf and dual specificity mitogen-activated protein kinase kinase (MEK) which finally activates ERK by phosphorylation. An alternative pathway of ERK activation in B cells uses Ras guanine nucleotide release protein 3 (RasGRP3), which is recruited to the plasma membrane by binding to DAG via its C1 domain. RasGRP3 catalyzes the switch to Ras-GTP, thereby initiating the ERK MAPK pathway (Li et al 1993, Buday et al 1994, Kumar et al 1995, Coughlin et al 2005, Roose et al 2007). B cell-specific deletion of ERK leads to reduced $B$ cell survival, proliferation and differentiation (Yasuda et al 2008, Yasuda et al 2011). There are at least two more MAPK pathways that are activated upon BCR stimulation including the p38 and c-Jun N-terminal kinase (JNK) pathway (Sutherland et al 1996).

DAG and $\mathrm{Ca}^{2+}$ also activate protein kinase $\mathrm{C}$ beta $(\mathrm{PKC} \beta)$, which mediates activation of an important transcription factor family called nuclear factor kappa of activated B cells (NF-kB) (Su et al 2002). These and other signaling pathways not mentioned here integrate in the nucleus (see Fig. 1), leading to a gene transcription program that decides the ultimate fate of the B cell - survival, proliferation, differentiation or apoptosis (Goodnow et al 2010). 


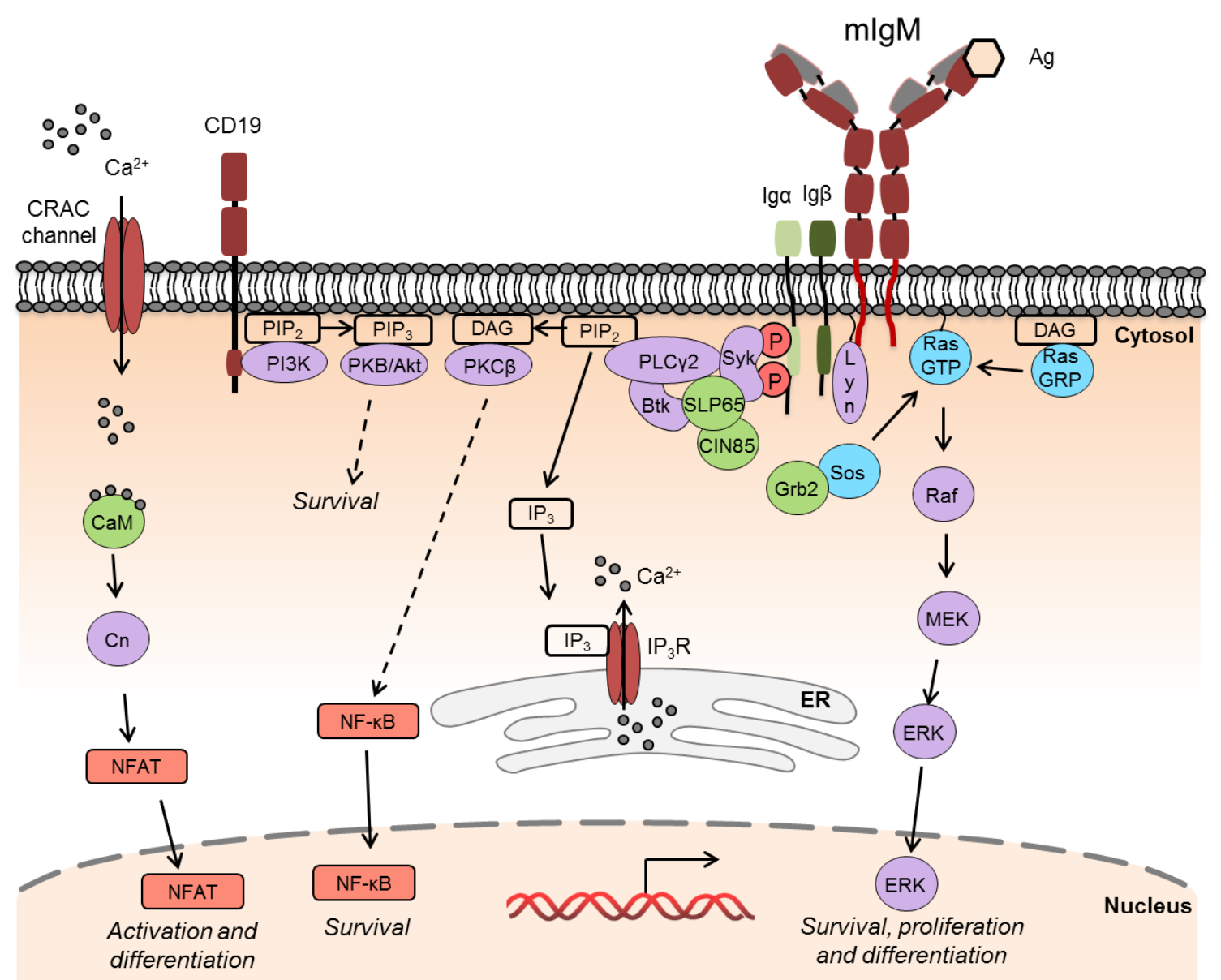

Figure 1: The diverse mlgM-BCR induced signaling pathways converge in the nucleus.

Schematic illustration of the main signaling pathways induced upon mlgM-BCR engagement by an antigen. BCR activation leads to phosphorylation of ITAMs in the $\lg \alpha / \lg \beta$ heterodimer by kinases like Lyn leading to recruitment of Syk to phosphorylated ITAMs and assembly of the $\mathrm{Ca}^{2+}$ initiation complex comprising SLP65, Btk and PLC $\gamma 2$. PLC $\gamma 2$ catalyzes the production of $\mathrm{IP}_{3}$ and $\mathrm{DAG}$ from $\mathrm{PIP}_{2} . \mathrm{IP}_{3}$ induces $\mathrm{Ca}^{2+}$ entry into the cytosol from the ER and subsequently via CRAC channels from the extracellular space, culminating in activation of the transcription factor NFAT. DAG promotes activation of PKC $\beta$ which leads to NF- $\mathrm{kB}$ activation and nuclear translocation. The ERK MAPK pathway can be activated by both Grb2-Sos and DAG-activated Ras-GRP. Co-stimulatory signals are provided by CD19 which recruits PI3K leading to activation of PKB/Akt. The cellular outcomes of these pathways which converge in the nucleus are specified. Cn-Calcineurin, CaMCalmodulin. Dashed lines represent indirect effects and solid lines represent direct effects. Purple-kinases and Cn phosphatase, green-adaptor proteins and $\lg \alpha / \lg \beta$ heterodimer, red-transcription factor, mlgM, CD19 and $\mathrm{Ca}^{2+}$ channels, blue-GEF/GTPase activity-containing proteins, black boxes-lipid messengers, beige-antigen $(\mathrm{Ag})$, grey dots-Ca ${ }^{2+}$, $P$-phosphorylation.

\subsection{Co-stimulatory signals support BCR signaling}

The intracellular signaling cascades kicked off by the BCR alone, in most cases are not sufficient for complete activation of the cell. This typically requires the activity of costimulatory receptors. Co-stimulatory signals may be provided by $T$ helper cells via the cluster of differentiation 40 (CD40)-CD40 ligand (CD40L) interaction at the immunological synapse between $B$ and $T$ cells. CD40 signaling mainly leads to the activation of NF-kB 
and thereby promotes cell survival, which can be beneficial in competitive environments like the germinal center (Tsubata et al 1993, Kawabe et al 1994, Mc-Heyzer Williams et al 2012). Additionally, the CD19-CD21-CD81 trimer of transmembrane proteins also plays an important role in B cell activation. CD21 is a complement receptor that binds antigenbound complement protein C3d. This leads to crosslinking of the entire CD19-CD21-CD81 complex to the BCR and phosphorylation of CD19, which is the signaling component of that complex (Fujimoto et al 2000). Phosphorylated tyrosine motifs in CD19 recruit many signaling proteins including Grb2, Vav and Phosphoinositide 3-kinase (PI3K) (Brooks et al 2004). PI3K for instance, increases the local concentration of phosphatidylinositol-3,4,5trisphosphate (PIP3) in the plasma membrane, which helps in recruitment of signaling proteins like Btk, PLC $\gamma 2$ and protein kinase B (PKB or Akt) via their Pleckstrin homology (PH) domains (Buhl and Cambier 1999, Jou et al 2002, Okkenhaug and Vanhaesebroeck 2003). PKB/Akt promotes cellular processes like cell survival, growth and differentiation (Pogue et al 2000, Herzog et al 2009) and thereby complements BCR signaling pathways in determining $\mathrm{B}$ cell fate.

\subsection{Germinal centers - sites for formation of B cell memory}

Upon activation, naïve B cells form secondary structures in lymphoid organs called germinal centers (GCs) (Silva and Klein 2015). One of the main processes that occur in a GC is class-switching to a different Ig isotype. The first vertebrates to acquire antibodies were the jawed cartilaginous fish which have mainly the IgM isotype. Ig isotype switching did not arise until much later in evolution with amphibians being the first vertebrates to switch Ig class, mainly to $\lg Y$. The $\lg Y$ isotype is believed to be the primordial ancestor of the mammalian $\lg G$ and $\lg E$ which are thought to have arisen from a gene duplication event (Mussmann et al 1996). IgY antibodies are present in amphibians, reptiles and also birds and are functionally similar to IgG (Flajnik 2002). Mammalian B cells undergo Ig class-switching to $\lg G$, IgE or IgA (Honjo et al 2002). Class-switching enables the immune system to mount responses that are specific for a certain class of pathogen. For instance soluble IgG bound to viral envelope proteins that are critical for viral entry into the cell, may prevent this virus from infecting other cells (Hangartner et al 2006). Likewise, helminth-specific $\operatorname{lgE}$ bound to Fc\&Rs on eosinophils induces secretion of cytotoxic granules that kill the helminth (Allen and Maizels 2011).

The second major process taking place in a GC is affinity maturation. B cells can increase the affinity of mlg molecules for their cognate antigen by undergoing somatic hypermutation (SHM) in the gene segments encoding for the Ig variable region (McHeyzer-Williams et al 1991, Honjo et al 2002). The GC environment is highly competitive and selective and therefore GC B cells that acquire mutations leading to lower affinity or loss of specificity rapidly undergo cell death due to engagement of the pro-apoptotic receptor Fas (Hao et al 2008). Higher affinity B cells which can take up more antigen for presentation to $\mathrm{T}$ follicular helper cells, get $\mathrm{T}$ cell help via CD40-CD40L interactions and 
therefore survive the $\mathrm{GC}$ selection process. After undergoing multiple rounds of selection and clonal expansion, the GC B cells eventually exit the GC as plasma cells or memory $B$ cells (Kawabe et al 1994, Batista and Harwood 2009, Mc-Heyzer Williams et al 2012, Silva and Klein 2015).

Recent studies have shown that the first cells to exit the GC are the low to medium affinity memory $\mathrm{B}$ cells that remain in circulation. These are then followed by high affinity long lived memory plasma cells that probably occupy a survival niche in the bone marrow (Weisel et al 2016). Besides IgG- and IgA-switched memory B cells, another population of isotype-switched $B$ cells that arise from the GC and whose molecular features are the least understood, are the IgE memory B cells.

\subsection{The biological role of $\lg E$}

$\lg \mathrm{E}$ is the least abundant $\lg$ isotype in the blood with a serum half-life of about 2 days in humans and about 5-12 hours in mice (Achatz et al 2010). It is a critical mediator of immediate hypersensitivity reactions and has hence gained a lot of limelight due to its clinical significance. IgE can be mainly found in the skin, alveoli and intestine where it coats the surface of mast cells and basophils via its interaction with FceRs expressed on these cells, also in the absence of residual antigen. The entry of antigens or allergens (for example pollen) into the body, and subsequent binding to this pre-formed IgE-FceR complex induces very rapid mast cell degranulation due to which allergies can present themselves within minutes. The response is mediated by Fc\&R-induced intracellular signaling pathways which culminate in secretion of granules containing histamine and/or secretion of cytokines and lipid mediators by these cells. The net result is vasodilation, bronchoconstriction and chemotaxis of leukocytes to the site, all contributing to the severe symptoms exhibited by allergy patients (Turner and Kinet 1999, Gould and Sutton 2008, Holgate and Polosa 2008, Wu and Shereens 2014). Treatment with anti-histamines and cortisol can provide temporary relief from these symptoms. However, the fact that lgEFc\&R complexes persist for long periods of time and sensitize mast cells to rapidly respond to antigens, demonstrates the need to treat allergies by targeting $\lg \mathrm{E}$. This has led to the clinical testing of monoclonal antibodies like Omalizumab, which can block IgEFceR interactions by neutralizing soluble lgE as therapy for allergies (Schulman 2001).

Besides playing a major role in allergic diseases, $\lg E$ also has a biological role, which does not attract as much attention. The physiological role of $\lg E$ is to clear extracellular pathogens like helminths, which cannot be phagocytosed due to their large size. This function is mediated by a complex consisting of helminth-bound $\lg E$ interacting with FceRs on the surface of mast cells, basophils and eosinophils. The direct mediators of pathogen killing in this case are the eosinophils which secrete granules containing cytotoxic proteins. Mast cells and basophils also contribute to pathogen clearance by facilitating activation and attraction of leukocytes to the site of infection (Gurish et al 2004, Mitre et al 
2006, Allen and Maizels 2011). Thus, the biologically relevant role of $\lg E$ in the immune system is to facilitate elimination of pathogens like helminths. Despite the clinical and biological significance of $\mathrm{lgE}$, it is not completely clear which B cell compartment maintains lgE memory.

\subsection{Is there a true IgE memory compartment?}

Until recently, it was debated whether true mlgE-expressing memory B cells even exist in vivo. This is mainly due to the scarce nature of mlgE-expressing cells and that accurate staining for mlgE on B cells is blurred by FceR-bound IgE on B cells and other immune cells. These issues were overcome by the use of mouse models where the $\varepsilon$ heavy chain locus was specifically targeted at the exons encoding the membrane isoform to express fluorescent proteins that would serve as definitive reporters for mlgE-positive B cells (Yang et al 2012, He et al 2013, Talay et al 2014). Collectively, these studies confirmed the presence of a transient pool of mlgE-positive GC B cells and a larger pool of mlgEpositive plasma cells in vivo. In addition, infection models using the model helminth

Nippostrongylus brasiliensis confirmed the presence of antigen-specific $\lg \mathrm{E}$ memory responses, as marked by high $\lg \mathrm{E}$ antibody titers upon re-challenge with the helminth (Talay et al 2014).

Unlike the mlgG-positive population, mlgE-positive GC B cells were shown to be short lived since they would either undergo rapid differentiation into plasma cells (Yang et al 2012) or were susceptible to apoptosis (He et al 2013). There has been a report of preferential expansion of mlgE-positive cells in the absence of Fas expression on GC B cells, indicating higher apoptosis susceptibility of these cells in a GC (Butt et al 2015). Due to the short life of mlgE memory B cells, the current belief in the field is that IgE memory resides in the mlgG1-positive $B$ cell population and that antigen recognition induces rapid differentiation of these cells into lgE-secreting plasma cells (He et al 2013, Yang et al 2012). In fact, analysis of the switch region in the $\varepsilon$ locus shows remnants of the $\gamma 1$ switch region, indicating a sequential isotype switch from $\gamma 1$ to $\varepsilon$ heavy chain. This feature has now been validated for human B cells as well. Nevertheless, direct isotype switching to $\lg \mathrm{E}$ has also been confirmed for both species, providing evidence towards the existence of an IgE memory fraction (He et al 2013, Ramadani et al 2016, Berkowska et al 2014).

In summary, an IgE memory compartment exists. However, the molecular basis behind IgE memory $B$ cell activation and differentiation remains largely unclear since most work on memory B cell biology has focused on mlgG-positive memory B cells. Studies on lgG memory $B$ cells clarified that the unique property of rapid activation and differentiation of memory B cells can be attributed to both BCR-extrinsic cellular factors as well as mlgGintrinsic signaling features, as discussed in the next sections (Wienands and Engels 2015). 


\subsection{How B cells remember - cellular features of memory B cell responses}

One of the hallmarks of the humoral immune system is a robust memory response to recurring infections that is generally marked by elevated serum IgG titers. This phenomenon is brought about by the circulating pool of memory $B$ cells that undergo rapid expansion and differentiation within 2-3 days of pathogen re-encounter (Dixon et al 1954, Yefenof et al 1986, Tangye et al 2003, Hebeis et al 2004). The bone marrow resident pool of long-lived memory plasma cells also contributes by constantly secreting protective antibodies and thus helping to maintain long term immunity (Radbruch et al 2006). The features that underlie the speed and efficiency of memory responses previously remained largely unclear. This is due to paucity of antigen-specific memory B cells and heterogeneity in the memory $B$ cell compartment, which makes identification and isolation of these cells cumbersome. More recently, the use of new sophisticated mouse models have led to a significant improvement in our understanding of how memory B cells function.

Gene expression analysis in memory B cells has revealed that they express high levels of factors that promote survival, proliferation and T cell co-stimulation, all of which contribute to prompt re-activation of these cells (Tangye et al 2003, Good and Tangye 2007, Klein et al 2003). Memory B cells were also found to express lower levels of the differentiation inhibitory protein Bcl6 as compared to their naïve counterparts (Bhattacharya et al 2009, Tomayko et al 2008). Additionally, mlgG1-expressing B cells that have undergone previous exposure to the antigen can mount a quantitatively superior antibody response due to antigen-dependent altered gene expression patterns (Kometani et al 2013). Higher expression of the cell surface molecules CD80 and Programmed death ligand 2, which are possibly involved in increasing $T$ cell co-stimulatory signals, was also indicated to be associated with increased differentiation of memory B cells into plasma cells (Zuccarino et al 2014). Finally, two independent studies collectively demonstrated that higher affinity IgG memory $B$ cells dominate the memory response and preferentially differentiate into plasma cells whereas the lower affinity $\operatorname{lgM}$ memory B cells re-enter the GC reaction (Dogan et al 2009, Pape et al 2011). Thus, a combination of aspects like BCR isotype, affinity and protein expression profile equip a memory $B$ cell with the ability to effectively clear the antigen and protect us from infections. The role of the BCR isotype in modulating memory $B$ cell responses was further pinned down to unique signaling mechanisms employed by mlgG-positive memory B cells as discussed next.

\subsection{BCR signal amplification via the immunoglobulin tail tyrosine (ITT) motif}

In contrast to the short cytoplasmic tail of mlgM that contains only three amino acids, $\mathrm{mlg} G$ and mlgE contain a longer cytoplasmic tail consisting of 28 amino acid residues. The amino acid sequences of these cytoplasmic tails show a high degree of conservation between different species, in particular for mlgG. Pioneering work in the 1990s indicated 
that the mlgG1 and mlgE tails are important for robust memory antibody responses of these $\mathrm{lg}$ isotypes. Mice lacking the cytoplasmic tail segment of either mlgG1 or mlgE were shown to produce reduced IgG1 or IgE antibody responses, respectively (Kaisho et al 1997, Achatz et al 1997). Moreover, mice genetically engineered to express mlgM-BCRs containing the mlgG1 cytoplasmic tail segment displayed robust IgG-like IgM antibody responses (Martin and Goodnow 2002).

Recently, it was demonstrated by our group that the longer cytoplasmic tails of mlgG and mlgE contain a conserved signaling motif now called the immunoglobulin tail tyrosine (ITT) motif (Engels et al 2009). ITT-like motifs can also be found in certain co-receptors in NK cells, T cells and other myeloid cells (Engels and Wienands 2011). The motif contains a highly conserved $\mathrm{YxN}$ amino acid core sequence ( $\mathrm{x}$ being any amino acid), which forms a perfect consensus binding site for the $\mathrm{SH} 2$ domain-containing adaptor protein Grb2. Upon mlgG-BCR stimulation, the ITT motif is phosphorylated by Syk, which leads to recruitment of Grb2 via its SH2 domain. Through its N-terminal SH3 domain, Grb2 recruits Btk to the $\mathrm{mlgG}$ tail thereby enhancing the activation of the $\mathrm{Ca}^{2+}$ initiation complex (see Fig. 2). This leads to increased $\mathrm{Ca}^{2+}$ flux in the cells and lowers the activation threshold for these cells (Engels et al 2009, Engels et al 2014).

The in vivo significance of the ITT motif was analyzed using a mouse model containing a single amino acid substitution of tyrosine to phenylalanine in the ITT motif at the $\gamma 1 \mathrm{~m}$ locus (referred to as mlgG1-YF mutant mice) (Lutz et al 2015). The formation of the mlgG1-positive memory compartment was completely normal in these mice. However, upon immunization, the mlgG1-YF mice displayed severely reduced serum lgG1 levels along with reduced numbers of IgG1-secreting plasma cells (see Fig. 2). Transfer of wildtype mlgG1 B cells into Rag $1^{\text {\% }}$ mice (which lack $T$ and B cells) led to enhanced IgG1 production when compared to transfer of mlgG1 B cells from the mlgG1-YF mice. Thus, the ITT promotes the production of $\lg G 1$ antibodies in a $\mathrm{T}$ cell-independent manner. Signaling via the mlgG-ITT is solely dependent on Grb2 since adoptive transfer of mlgGexpressing cells into Rag $1^{-/}$mice enhanced IgG production in a Grb2 dependent manner (Engels et al 2014) which is in congruence to a previous study that found reduced lgG responses in Grb2 deficient mice (Ackermann et al 2011). Lastly, the ITT also promotes the long term survival of mlgG1 cells as more antigen-specific mlgG1 memory B cells were detected 250 days after immunization. Therefore, the ITT motif promotes survival and revival of memory $B$ cells, thereby acting as a molecular determinant of memory responses. 
A

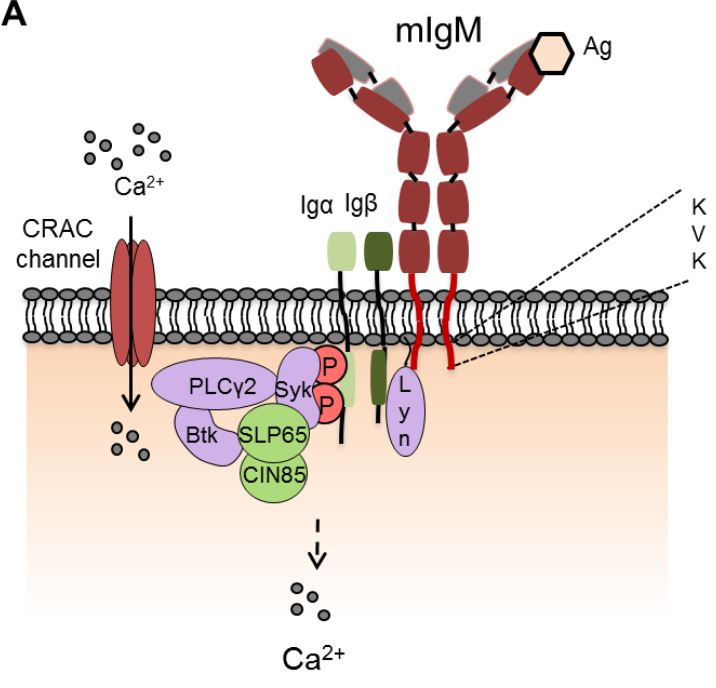

B

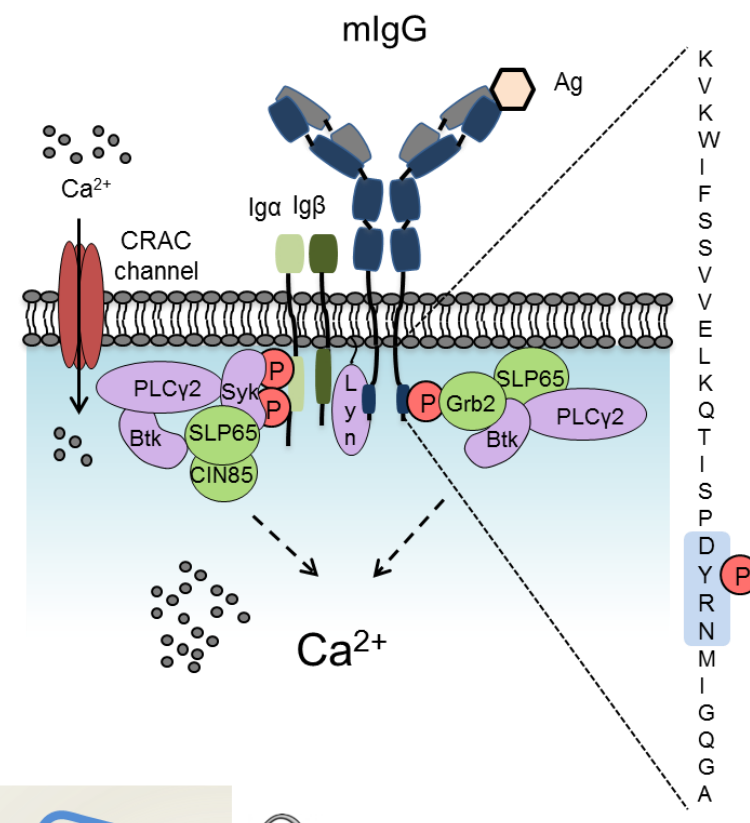

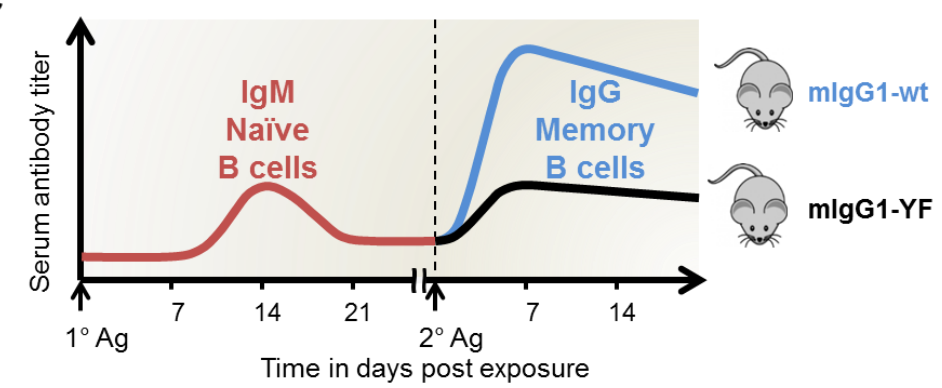

Figure 2: BCR signal amplification by the mlgG-ITT motif alters IgG memory responses. (A) The short cytoplasmic tail of the mlgM is depicted. mlgM-BCR activation leads to ITAM signaling induced $\mathrm{Ca}^{2+}$ flux and ERK activation as described in Fig. 1. (B) The longer cytoplasmic tail of mlgG (mouse $\gamma 2$ am sequence shown here) is depicted with the ITT motif highlighted in blue. mlgG-BCR engagement activates the canonical ITAM signaling pathways as described for mlgM-BCRs. In addition, mlgG-ITT phosphorylation leads to recruitment of the Grb2-Btk module to the mlgG tail thereby augmenting $\mathrm{Ca}^{2+}$ flux via mlgG-BCRs. (C) An illustrative time course of antibody responses upon primary $\left(1^{\circ}\right)$ and secondary $\left(2^{\circ}\right)$ infection with a pathogen. Primary responses are marked by naïve $B$ cells that mount low to moderate serum lgM titers (in red), whereas secondary responses involve memory B cell-mediated high serum IgG titers (in blue). Mice with a wild-type mlgG1-BCR mount normal IgG1 responses whereas mlgG1-ITT mutant mice (mlgG1-YF mice) mount lgG1 responses which are more reminiscent of IgM responses (in black). Dashed lines represent indirect effects and solid lines represent direct effects. Purple-kinases, green-adaptor proteins and $\lg \alpha / \lg \beta$ heterodimer, redmlgM and $\mathrm{Ca}^{2+}$ channel, blue-mlgG, beige-antigen $(\mathrm{Ag})$, grey dots- $\mathrm{Ca}^{2+}, \bigodot_{-}$-phosphorylation.

\subsection{Additional molecular features of the mlgG tail}

Besides the ITT, a so called SSVV (single letters for amino acids) motif was identified in the cytoplasmic tail of $\mathrm{mlgG}$ and shown to enhance IgG-BCR clustering via recruitment of a protein called SAP97 (Liu et al 2012). However, a mutant SSVV motif in the mlgG tail does not have any effect on $\mathrm{Ca}^{2+}$ signaling in $\mathrm{B}$ cells (Engels et al 2014). Moreover, since immunodeficient mice that were reconstituted with SAP97-deficient $\mathrm{B}$ and $\mathrm{T}$ cells did not 
display any defects in IgG responses, the motif probably does not play a role in enhancing memory responses in vivo (Hawkins et al 2013). More recent studies into the role of the mlgG tail indicated that positively charged amino acid residues in its cytoplasmic tail anchor it to the plasma membrane via interactions with acidic phospholipids, the functional role for which remains however unknown (Chen et al 2015). Finally, the BCR is also known to sense physical properties like the stiffness of the antigen to initiate B cell activation (Wan et al 2013). In connection to this, it was shown that mlgG-BCRs possess a lower threshold for mechanical force-induced activation as compared to mlgM-BCRs, which may also contribute to their improved activation capabilities (Wan et al 2015).

\subsection{How does the ITT influence mlgE-BCR signaling?}

Mice that cannot express mlgE due to targeted deletion of the transmembrane region have almost undetectable levels of serum IgE upon helminth infection (Achatz et al 1997). This indicates that even though transient, a mlgE-BCR-positive phase exists and is absolutely required for $\lg E$ production. Hence, mlgE-BCR signaling probably plays a crucial role in the activation of $\lg E$ memory $B$ cells and regulation of serum $\lg E$ responses. The mlgE cytoplasmic tail from both mice and humans contain the ITT motif, which may contribute to mlgE-BCR signaling. There is only one mlgE isoform in mice, whereas human mlgE exists in two membrane isoforms referred to as long and short (Zhang et al 1992, Batista et al 1995, Batista et al 1996). The long isoform contains 52 additional amino acid residues $\mathrm{N}$-terminal of the transmembrane region, termed the extracellular membrane-proximal domain (EMPD). Both isoforms are expressed on human B cells (Zhang et al 1992, Batista et al 1995) and in fact a monoclonal antibody against the EMPD called Quilizumab is currently under trial in the clinics to treat allergies (Chen et al 2002, Gauvreau et al 2014). Since both isoforms of human mlgE have an identical cytoplasmic tail, they both contain an ITT motif which may contribute to $\lg E$ memory responses. IgE-mediated allergic responses are a major health concern and insights into the molecular signaling mechanisms of mlgE-BCRs could prove to be relevant to finding ways of preventing the activation/expansion of IgE memory B cells. Therefore, to better understand the biology behind IgE memory responses, this work has focused on the signaling pathways induced downstream of mlgE-BCRs. 


\subsection{Scope of the thesis}

The biology of mlgE-expressing B cells remains elusive despite its relevance in allergic diseases. The mlgE cytoplasmic tail contains the evolutionarily conserved ITT motif. The ITT was previously shown to enhance BCR signaling and IgG responses in vivo in the context of mlgG-BCRs (Lutz et al 2015, Engels et al 2014, Engels et al 2009). However, the molecular details of mlgE-ITT signaling remained unknown. The scarcity of mlgEpositive cells makes it very difficult to analyze mlgE-BCR signaling in vivo using mouse models or ex vivo using isolated human blood B cells. Therefore, I utilized human B cell lines to express the short isoform of human IgE and analyze mlgE-BCR induced signaling pathways. In this study, I addressed the following questions regarding the functional properties of mlgE-BCRs -

- What is the functional role of the mlgE-ITT in BCR proximal signaling processes?

- Which signaling proteins function downstream of the mlgE-ITT?

- Can the mlgE-ITT mediate signal transduction independently of the $\lg \alpha-\lg \beta$ heterodimer? 


\section{Results}

\subsection{The ITT increases the sensitivity of mlgE-BCRs by utilizing the adaptor proteins Grb2 and Grap.}

The ITT motif is highly conserved at the amino acid level in higher vertebrate species where it is present in the cytoplasmic tail of $\mathrm{mlgG}$, mlgE and $\mathrm{mlgY}$ (Fig. 3A). In order to analyze the functional properties of the ITT motif, I introduced the mlg heavy chains of different isotypes into the human B cell line DG75 by retroviral transduction. The expressed mlg heavy chains pair with the endogenous kappa light chain of DG75 cells and the $\lg \alpha-\lg \beta$ heterodimer to form a fully functional BCR on the cell surface. I used the mouse $\gamma 2 a m$ (a mlgG subclass), human $\varepsilon m s$ (short isoform of mlgE) and a $\gamma 2 a m-u m$ chimera where the mouse $\gamma 2$ am cytoplasmic tail was replaced with that of um (mlgY) from the lizard Anolis carolinensis. The BCRs were expressed in either WT or tyrosine to alanine (YA) ITT-mutant configurations to analyze the effect of the ITT on BCR signaling (Fig. 3B). DG75 cells also express an endogenous mlgM-BCR, whose signaling properties should be identical irrespective of which exogenous BCR is expressed by the transfected cells, and therefore this served as an internal control.

Stimulation of the WT ITT-containing BCRs considerably enhanced $\mathrm{Ca}^{2+}$ flux in cells as compared to the ITT-YA BCRs in all isotypes analyzed (Fig. 3C-E). Similar levels of signaling by the endogenous mlgM-BCRs demonstrated that the signaling capabilities of the cells were preserved in case of all transfectants (Fig. 3F-H). Furthermore, surface expression of the BCRs was analyzed and demonstrated that the ITT-YA mutant BCRs were expressed at the same or even slightly higher levels than the WT BCRs (Fig. 3I-K). This eliminated the possibility of expression-related differences in $\mathrm{Ca}^{2+}$ signaling between WT and ITT-YA BCRs. These results demonstrate that the ITT is the primary signal amplification device present in memory-type BCRs of higher vertebrate species (result published in Engels et al 2014).

The ITT-YA substitution reduced the $\mathrm{Ca}^{2+}$ signaling of mlgE-BCRs to levels similar to those seen on stimulation of mlgM-BCRs (Fig. 3). This indicated that the ITT might be the key signal amplification device of the mlgE cytoplasmic tail. To test this notion, I generated a variant of the mlgE molecule which had a short cytoplasmic tail consisting of only four amino acids, namely MVQR (single letter amino acid code, referred to as tailless from here on), that were retained for membrane-anchoring of the molecule (Fig. 4A). The tailless mlgE-BCR displayed lower expression as compared to the WT and ITT-YA mlgEBCRs in DG75 B cells (Fig. 4B). However, upon mlgE-BCR stimulation, the $\mathrm{Ca}^{2+}$ kinetics were similar for the ITT-YA and the tailless mlgE-BCRs (Fig. 4C). The endogenous mlgMBCR-induced signaling was similar for all transfectants (Fig. 4D). 
Results

A

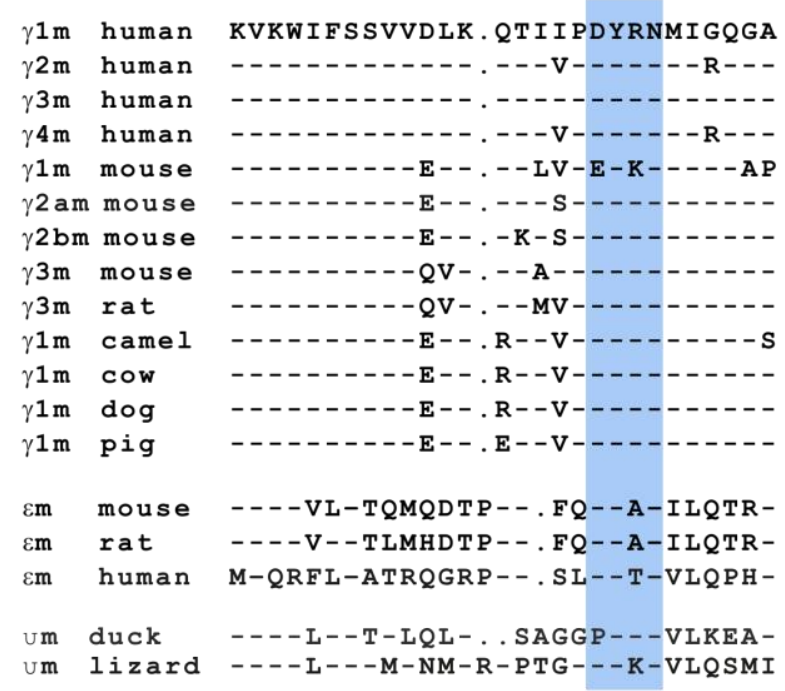

B

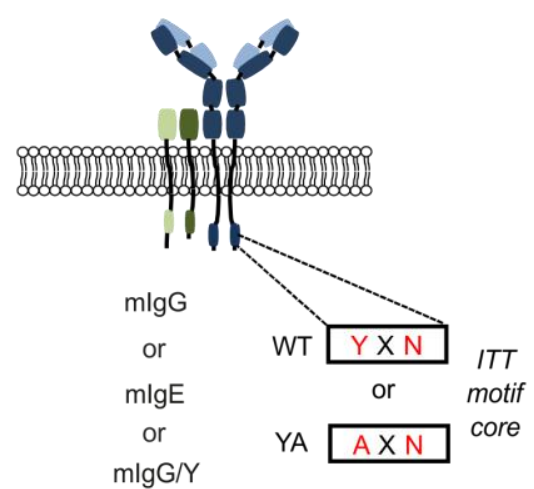

C

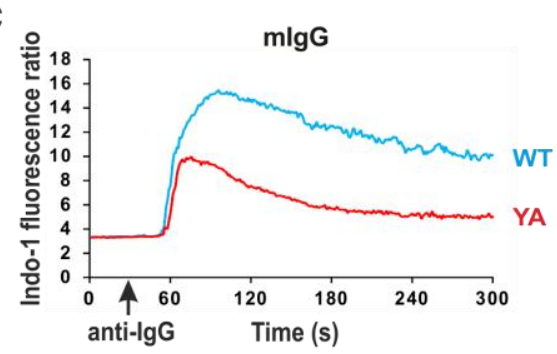

D

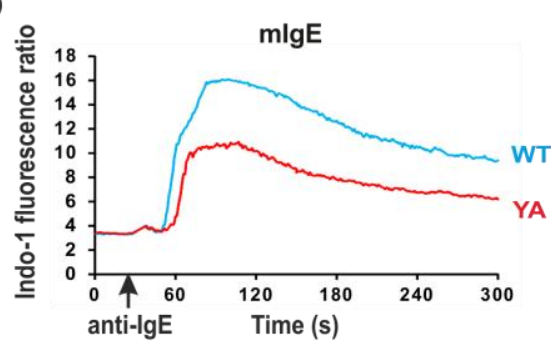

E

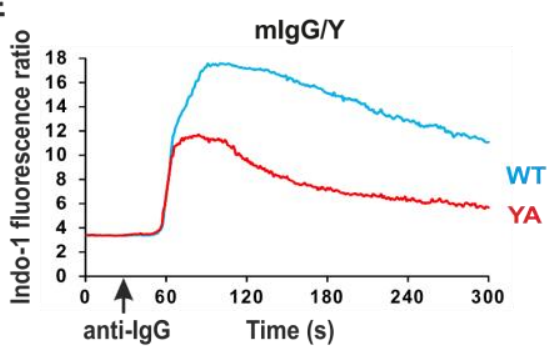

$\mathbf{F}$

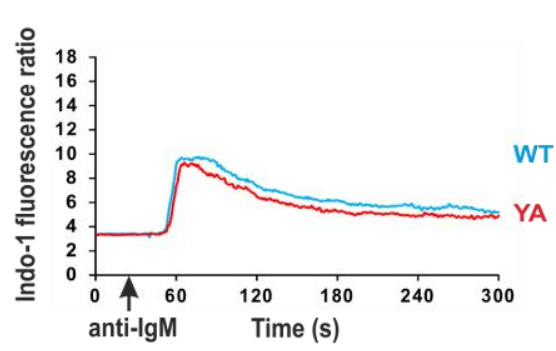

G

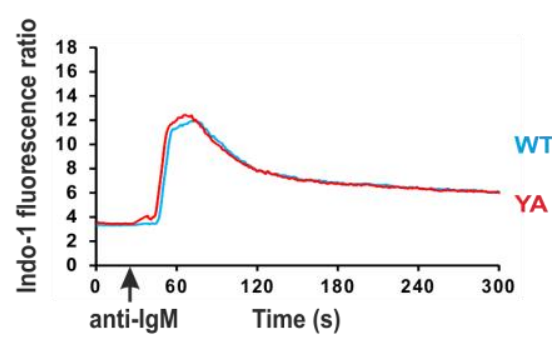

H

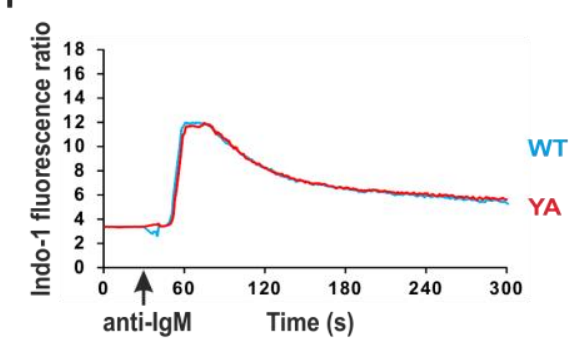

I

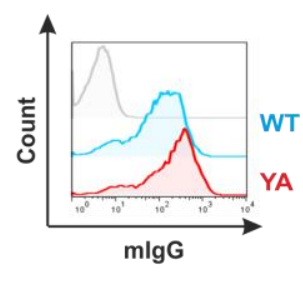

J

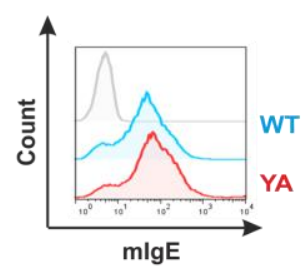

K

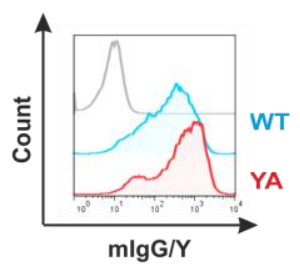

Figure 3: The ITT is the primary signal amplification module in memory type-BCRs.

(A) Amino acid sequence alignment of the cytoplasmic tail segments of $\mathrm{mlgG}(\gamma \mathrm{m}), \mathrm{mlgE}(\mathrm{\varepsilon m})$ and $\mathrm{mlgY}(\mathrm{um})$ from various higher vertebrate species. The conserved ITT motif is highlighted in blue. (B) Schematic illustration of the experimental setup used to analyze memory type-BCRs. The mlgG, mlgE and mlgG/Y BCRs were expressed using retroviral transduction in wild-type DG75 human B cells in WT or inactivated ITT-YA configuration. The $\mathrm{Ca}^{2+}$ mobilization kinetics of the mlgG-ITT (C) and mlgY-ITT (E) upon stimulation with 10 $\mu \mathrm{g} / \mathrm{ml}$ anti-mouse-IgG $\mathrm{F}(\mathrm{ab})_{2}{ }_{2}$ fragments or the $\mathrm{mlgE}-\mathrm{ITT}$ upon stimulation with $10 \mu \mathrm{g} / \mathrm{ml}$ anti-human-IgE antibody (D). Stimulation with $20 \mu \mathrm{g} / \mathrm{ml}$ anti-human-IgM $F(a b)_{2}{ }_{2}$ fragments as control for cells expressing mlgG- (F), mlgE- (G) and mlgG/Y-BCRs (H). Surface expression of WT and ITT-YA mutant mlgG- (I), mlgE(J) and $\mathrm{mlg} G / Y-B C R s(K)$. Data are representative of at least two independent experiments. 
A

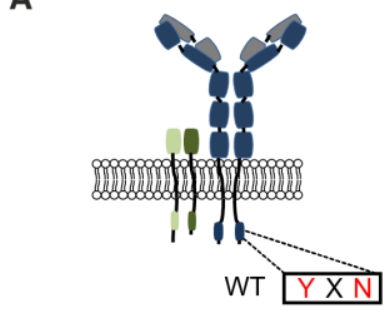

C
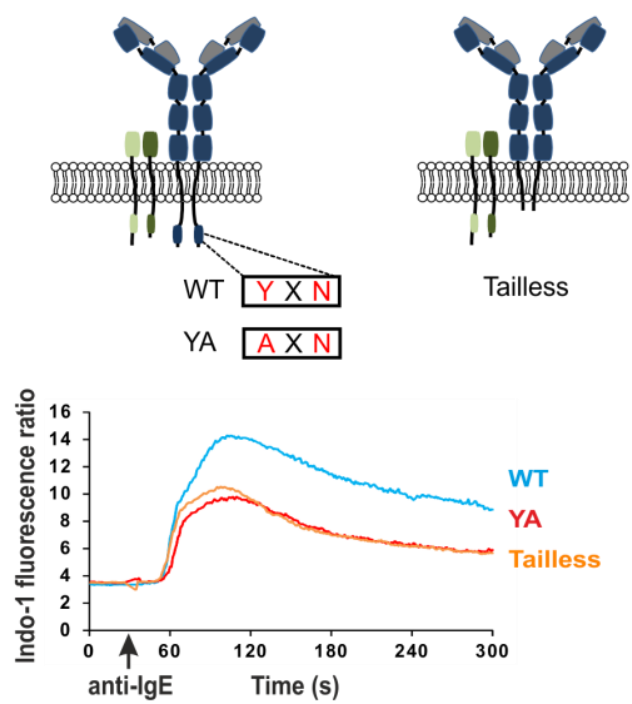

B

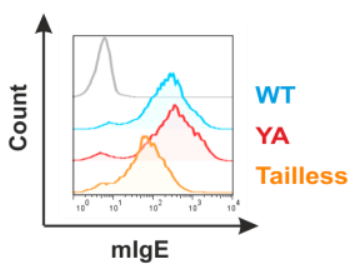

D

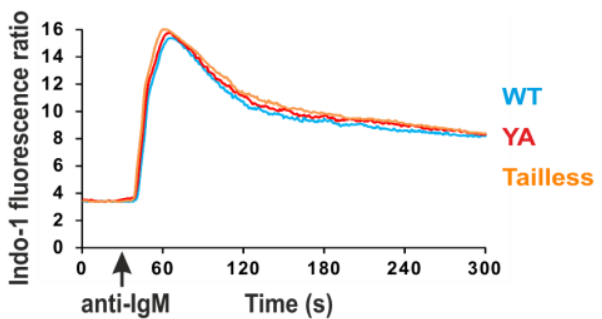

Figure 4: The ITT is the sole signaling motif in the mlgE cytoplasmic tail.

(A) Schematic representation of wild-type DG75 cells expressing the WT, ITT-YA mutant or tailless mlgEBCRs. The tailless mlgE has a short cytoplasmic tail with only four $\mathrm{N}$-terminal amino acids namely, MVQR. (B) Surface expression of the mlgE-BCR variants. $\mathrm{Ca}^{2+}$ mobilization kinetics of the cells upon stimulation with $10 \mu \mathrm{g} / \mathrm{ml}$ anti-human-IgE antibody (C) or $20 \mu \mathrm{g} / \mathrm{ml}$ anti-human-IgM F(ab)' ${ }_{2}$ fragments as control (D). Data are representative of three independent experiments.

This experiment gave some relevant insights into mlgE-BCR signaling. First, the mlgE molecule lacking the cytoplasmic tail is not as efficiently transported to the cell surface as compared to mlgE with an intact cytoplasmic tail. Secondly, the ITT turned out to be the only signal amplification device in the mlgE cytoplasmic tail since the tailless variant has a signaling capacity similar to the ITT-YA mlgE-BCR.

Next, I turned my focus on further analysis of the human mlgE-BCR and its signaling mechanisms which were not well understood. I first looked at how signal amplification by the ITT affects the activation threshold of mlgE-expressing B cells. Upon titrating the amount of stimulating antibody, one can see that BCR signaling via the mlgE-ITT required approximately 5 fold less antigen (stimulating antibody in this case) than the ITT-YA mutant to induce comparable $\mathrm{Ca}^{2+}$ mobilization kinetics (Fig. 5A, compare green curve for WT with purple curve for YA mutant). Again, endogenous mlgM-BCR-induced $\mathrm{Ca}^{2+}$ flux is the same for cells expressing the ITT variants (Fig. 5B). I also analyzed the effect of the ITT on the phosphorylation of the MAP kinase ERK, a protein that plays a critical role in the activation of $B$ cells (Yasuda et al 2011). ERK phosphorylation was similar at high antigen concentrations, however, under sub-optimal stimulation conditions, the ITTcontaining mlgE-BCR more efficiently activated ERK than the YA-mutant counterpart (Fig. $5 \mathrm{C})$. Combined, these data show that the ITT lowers the activation threshold of signaling pathways downstream of the mlgE-BCR, thereby equipping mlgE-expressing B cells with the capability of being efficiently activated at low antigen concentrations. 
Results

A
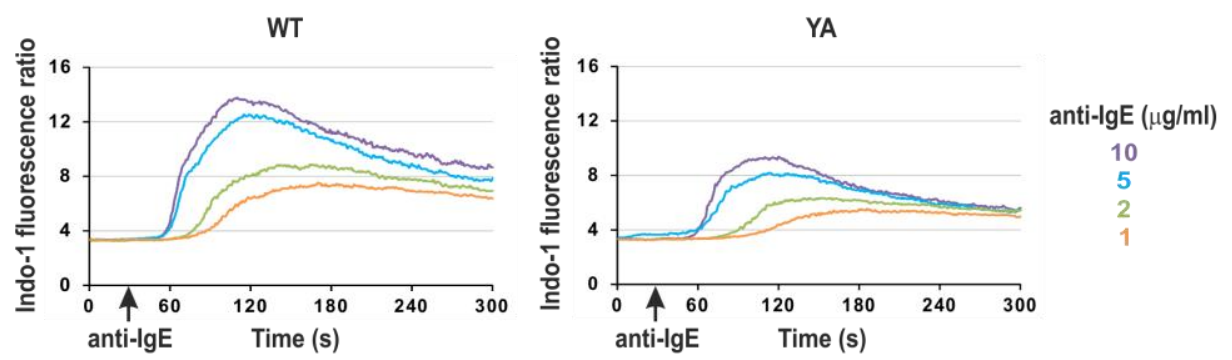

B

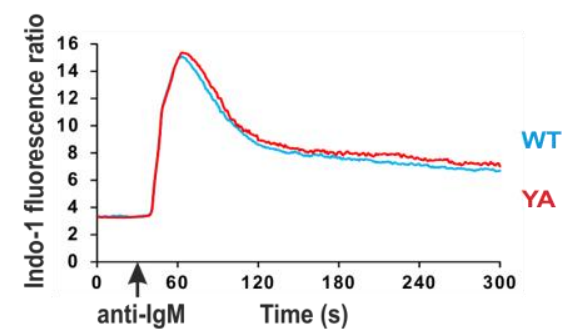

C

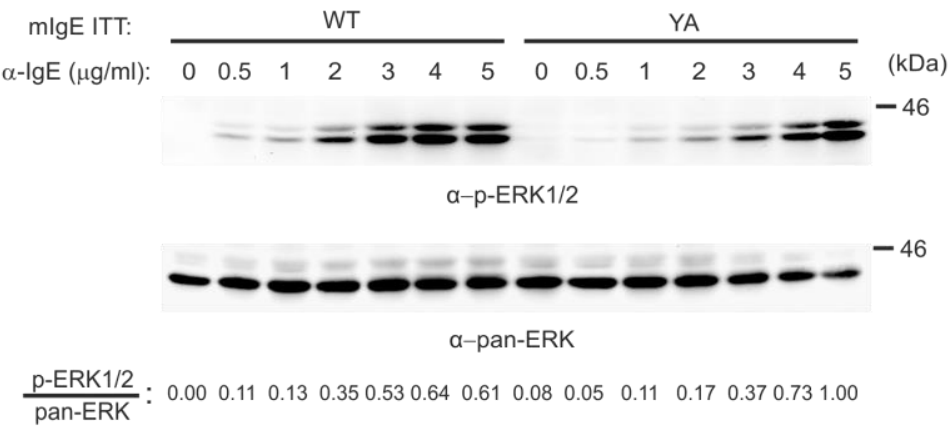

Figure 5: The ITT increases the sensitivity of mlgE-BCRs

(A) DG75 B cells were used to measure $\mathrm{Ca}^{2+}$ mobilization kinetics of the WT and ITT-YA mutant mlgE-BCRs upon stimulation with the indicated concentrations of anti-human-lgE antibody. (B) mlgM-BCR induced $\mathrm{Ca}^{2+}$ mobilization of the same cells using $20 \mu \mathrm{g} / \mathrm{ml}$ anti-human-IgM $F(a b)_{2}{ }_{2}$ fragments. (C) Cell lysates were prepared after stimulation of WT and ITT-YA mutant mlgE-BCRs with indicated concentrations of anti-humanIgE antibody. ERK phosphorylation was analyzed by SDS-PAGE and immunoblotting with antibodies against phospho-ERK ( $p$-ERK) or total ERK (pan-ERK). Relative levels of phospho-ERK to total ERK were quantified and are given at the bottom. Data are representative of two $(A, B)$ or three $(C)$ independent experiments.

The next question that I addressed was to find which tyrosine kinase phosphorylates the ITT motif in mlgE. The ITAMs present in the $\lg \alpha / \lg \beta$ heterodimer are thought to be initially phosphorylated by Src family kinases such as Lyn (Rolli et al 2002), which leads to recruitment of Syk to doubly phosphorylated ITAMs (Johnsen et al 1995, Kurosaki et al 1995). Syk can then activate downstream signaling molecules and also perhaps contribute to a certain degree to ITAM phosphorylation (Rolli et al 2002). To test which of the two BCR-associated kinases are required for ITT phosphorylation, I treated DG75 cells expressing a WT mlgE-BCR with the Src kinase inhibitor PP1 or the Syk inhibitor Bay61-3606. Next, I stimulated the mlgE-BCR with a biotinylated anti-lgE antibody and used this antibody to purify mlgE molecules. I found that inhibition of Syk abrogated ITT phosphorylation (Fig. 6A). Lyn inhibition also inhibited ITT phosphorylation but this is probably due to lack of Syk recruitment to the BCR since Lyn functions upstream of Syk in 
A

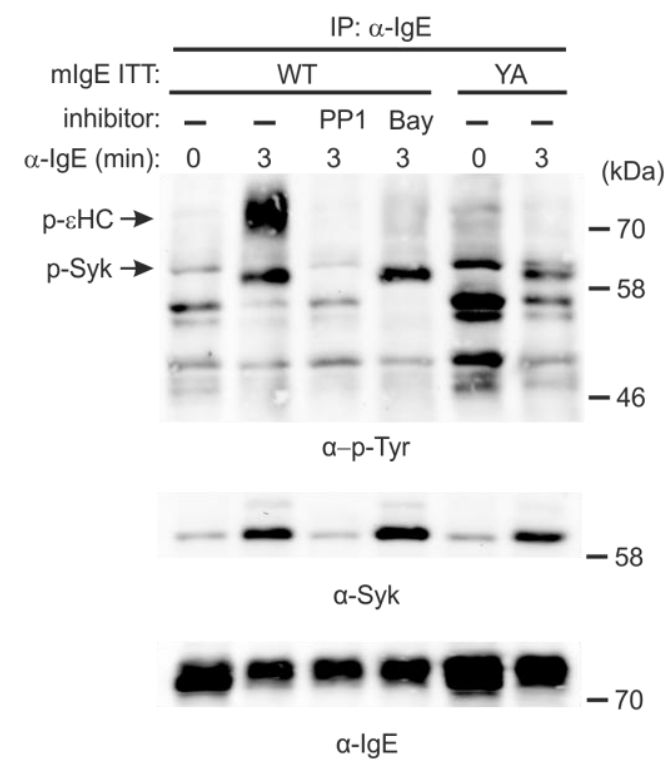

B

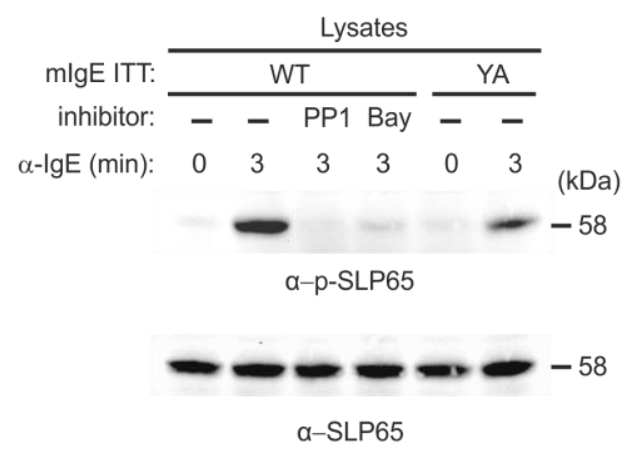

Figure 6: The human mlgE-ITT is phosphorylated by the tyrosine kinase Syk.

(A) DG75 cells expressing a WT mlgE-BCR were treated for 30 min with $30 \mu \mathrm{M}$ Src kinase inhibitor PP1 or 30 $\mu \mathrm{M}$ Syk kinase inhibitor Bay61-3606 or left untreated (-). The cells were then left unstimulated (0) or stimulated for $3 \mathrm{~min}(3)$ with $10 \mu \mathrm{g} / \mathrm{ml}$ anti-human-lgE-Biotin antibody. ITT-YA mlgE-BCR-expressing cells were used as control. Cell lysates were prepared and the BCR complex was purified using streptavidin beads and analyzed by SDS-PAGE and immunoblotting. mlgE-ITT-phosphorylation was detected using the anti-pTyr100 antibody. The blot was also probed with anti-Syk and anti-IgE antibodies. (B) Cell lysates from (A) were separately analyzed by SDS-PAGE and immunoblotting for phospho-SLP65 and total SLP65 was used as loading control. Data are representative of three independent experiments.

the signaling cascade (Kurosaki et al 1995, Keshvara et al 1998). To confirm the efficiency of inhibitor activity, I analyzed SLP65 phosphorylation (a downstream target in the signaling cascade) in cell lysates and found that both inhibitors were functional (Fig. $6 \mathrm{~B})$.

Having established that the mlgE-ITT is phosphorylated by Syk, the next step was to find which effector molecule is responsible for the ITT-mediated signal amplification of mlgEBCRs. The phospho- $Y \times N$ core motif of the ITT is a perfect consensus binding site for the $\mathrm{SH} 2$ domain of the adaptor molecule Grb2 (schematically depicted in Fig. 7A). It has been previously published that Grb2 is the main effector molecule for signal amplification by the mlgG-ITT. This process is dependent on the N-terminal SH3 domain of Grb2, which recruits Btk to the mlgG-BCR signaling complex (Engels et al 2014). Hence, I used a derivative of DG75 cells made deficient for GRB2 by gene targeting (DG75 GRB2 KO) (cells generated by Dr. Johannes Lutz). I expressed the mlgE-BCR variants in these cells to test if the mlgE-ITT also requires Grb2 for signal amplification. As control, I analyzed mlgG-BCR signaling in the same cellular background. As expected, the mlgG-ITT completely lost its signal amplification capabilities in GRB2 KO cells (Fig. 7B). Although, the relative degree of mlgE-ITT signal amplification was reduced in the GRB2 KO compared to wild-type DG75 cells, it was not completely eliminated (Fig. 7C). 
A

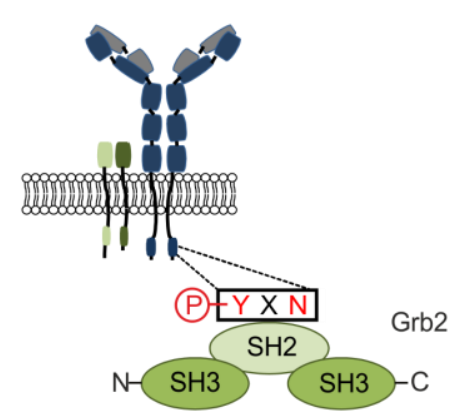

B

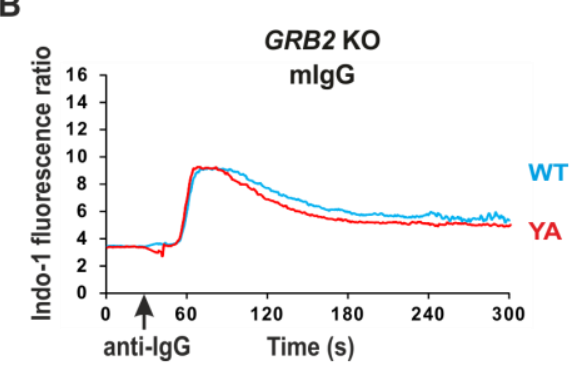

C

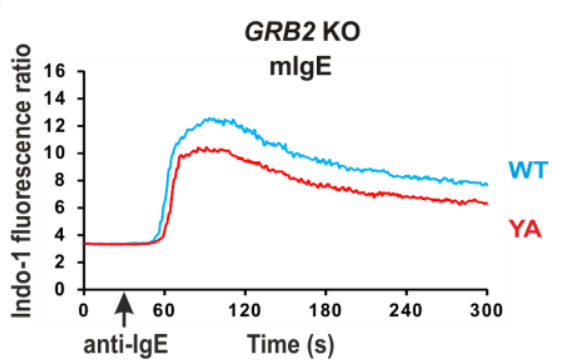

D

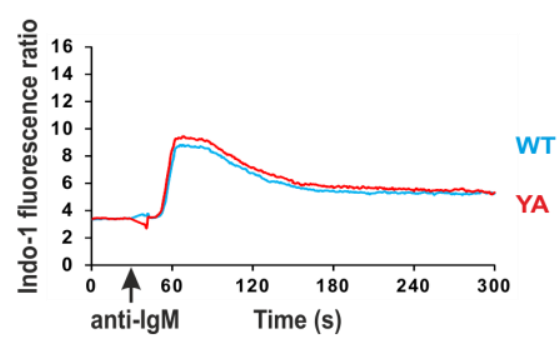

E

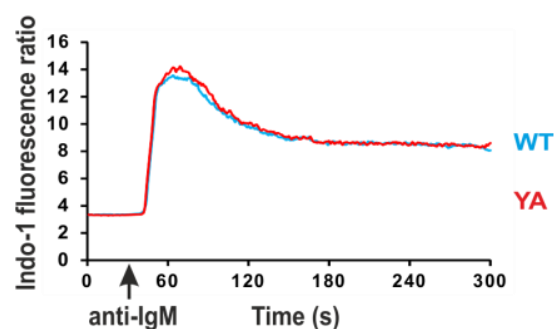

$\mathbf{F}$

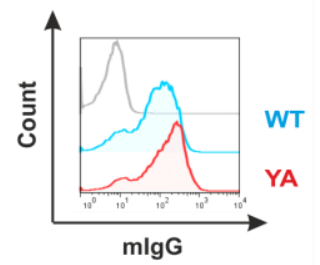

G

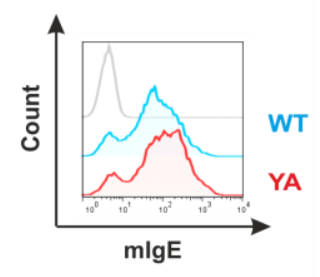

Figure 7: The human mlgE-ITT is partly functional even in the absence of the adaptor protein, Grb2.

(A) Schematic illustration highlighting the core residues in the ITT motif which form a binding site for the SH2 domain of Grb2. DG75 GRB2 KO cells were therefore used to analyze ITT function. $\mathrm{Ca}^{2+}$ flux in GRB2 KO cells expressing WT or ITT-YA mutant mlgG- or mlgE-BCRs, stimulated with $10 \mu \mathrm{g} / \mathrm{ml}$ anti-mouse-lgG F(ab)' ${ }_{2}$ fragments (B) or $10 \mu \mathrm{g} / \mathrm{ml}$ anti-human-lgE antibody (C) respectively. $\mathrm{Ca}^{2+}$ flux following stimulation with 20 $\mu \mathrm{g} / \mathrm{ml}$ anti-human-lgM $\mathrm{F}(\mathrm{ab})^{\prime}{ }_{2}$ fragments for cells expressing mlgG-BCRs (D) or mlgE-BCRs (E). Surface expression of the mlgG-BCR (F) and mlgE-BCR (G) variants. Data are representative of at least two independent experiments.

This would imply that another protein can partially compensate for the loss of Grb2 in the context of the mlgE-ITT. Endogenous mlgM-BCR induced $\mathrm{Ca}^{2+}$ signaling (Fig. 7D,E) and relative surface expression of the ITT variants (Fig. 7F,G) were comparable in the mlgGand mlgE-BCR-expressing cells.

Since the core of the ITT motif contains a conserved asparagine $(\mathrm{N})$ residue at the +2 position relative to the phosphorylated tyrosine, I replaced this residue with an alanine, generating a mlgE-NA mutant (Fig. 8A). Upon expression of the WT, YA and NA mutant mlgE-BCRs in wild-type DG75 B cells (Fig. 8B), I observed that the NA mutant responds like the YA mutant in that ITT signal amplification is completely abolished (Fig. 8C). As always, endogenous mlgM-BCR signaling was similar for all transfectants (Fig. 8D). This shows that both the tyrosine and the asparagine residues in the ITT motif core are critical for binding of mlgE-ITT effector molecules. 
A

C

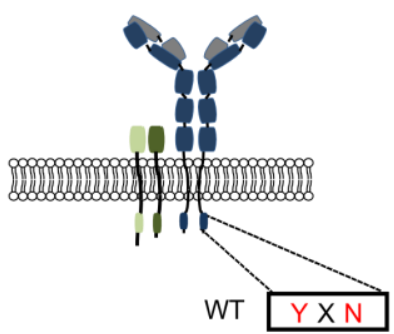

YA $\mathrm{AXN}$

NA YXA

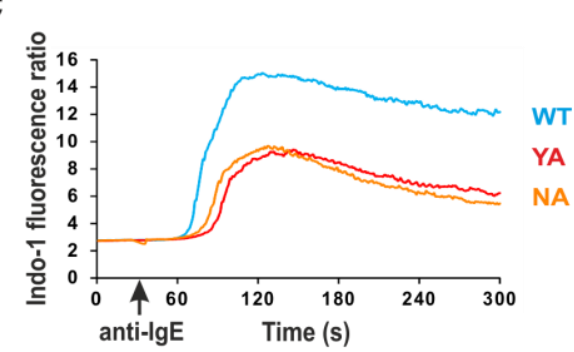

B

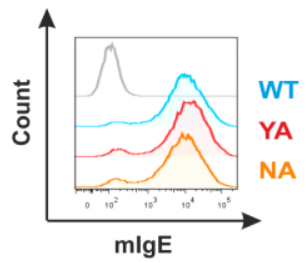

D

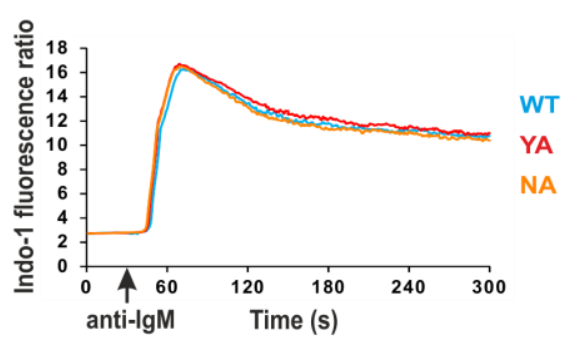

Figure 8: Signal amplification by human mlgE-BCRs depends on the asparagine residue in the ITT motif core.

(A) Schematic illustration of the experimental setup used to analyze the mlgE-ITT motif core. Wild-type DG75 cells were transfected with mlgE-BCRs containing a WT, ITT-YA mutant or ITT-NA (asparagine to alanine substitution) mutant. (B) Surface expression of the mlgE-BCR variants. $\mathrm{Ca}^{2+}$ mobilization kinetics of the cells upon stimulation with $10 \mu \mathrm{g} / \mathrm{ml}$ anti-human-IgE antibody (C) or $20 \mu \mathrm{g} / \mathrm{ml}$ anti-human-lgM F(ab)'2 fragments (D). Data are representative of three independent experiments.

To obtain a more comprehensive view of mlgE-ITT interaction partners, I used a synthetic biotin-tagged phospho-peptide encompassing the ITT motif and the surrounding amino acids to affinity purify proteins that bind to the ITT in a phosphorylation dependent manner (Fig. 9A). The mIgE-ITT interaction partners purified from SILAC-labeled B cell lysates were identified via mass spectrometry (done in collaboration with the Proteomics Core Facility of University Medical Center Göttingen). A non-phosphorylated peptide was used as a negative control so that proteins that specifically bind to the phosphorylated mlgE-ITT alone are enriched (Fig. 9B). After applying a 2.0 fold enrichment ratio cut-off for proteins that fulfilled the significance conditions, 22 proteins were found to associate with the phosphorylated mlgE-ITT (Fig. 9C,D). It is important to note that these proteins include both direct and indirect interaction partners. Interestingly, the Grb2 related adaptor protein, Grap was purified along with the mlgE-ITT and was amongst the proteins that showed the highest enrichment (highlighted in red in Fig. 9D). Grap was previously identified as a direct interaction partner of the mlgG-ITT as well. However, it was demonstrated to be incapable of amplifying BCR signaling in the case of the mlgG-ITT, which was solely dependent on Grb2 (Engels et al 2014, Fig. 7B). Nevertheless, if one combines the knowledge that Grb2 and Grap share a high degree of homology (schematically represented in Fig. 10A) and that mlgE-ITT signal amplification depends on the YxN ITT core motif, Grap would make an ideal candidate as a second effector molecule of the mlgE-ITT. 
Results

A Biotin-RPQTSLDYTNVLQPHA

B

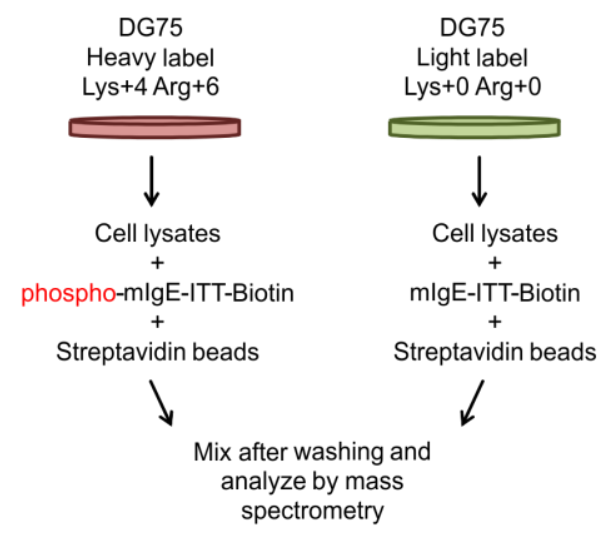

\section{(P)}

C

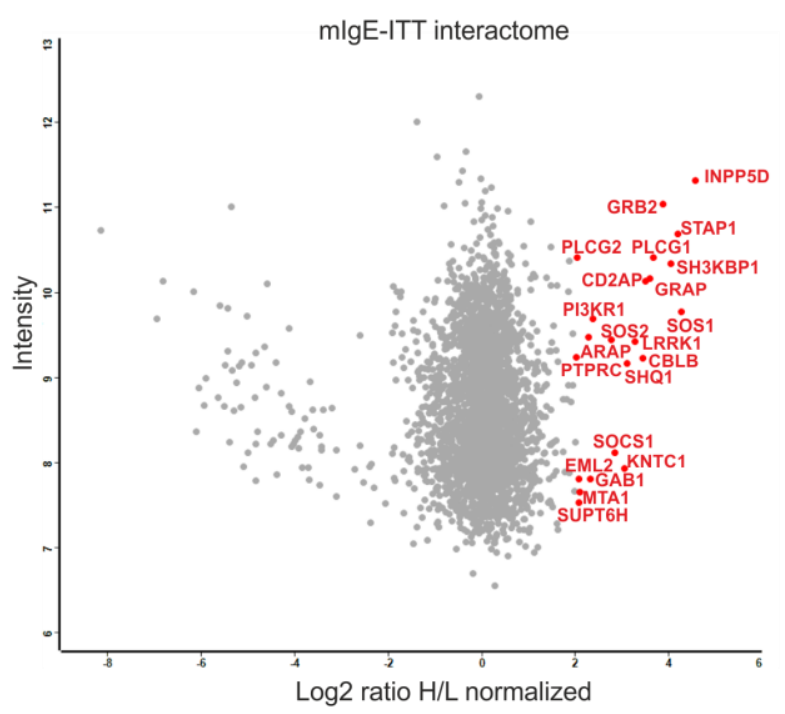

D

\begin{tabular}{|c|c|c|c|c|}
\hline $\begin{array}{c}\text { Ratio } \mathrm{H} / \mathrm{L} \\
\text { normalized }\end{array}$ & No. Peptides & Gene name & Protein ID & Protein name \\
\hline 4.5656 & 99 & INPP5D & Q92835 & Phosphatidylinositol 3.4.5-trisphosphate 5-phosphatase 1 \\
\hline 4.25626 & 63 & sos1 & Q07889 & Son of sevenless homolog 1 \\
\hline 4.19077 & 30 & STAP1 & Q9ULZ2 & Signal-transducing adaptor protein 1 \\
\hline 4.02751 & 39 & SH3KBP1 & Q96B97 & SH3 domain-containing kinase-binding protein 1 \\
\hline 3.86898 & 23 & GRB2 & P62993 & Growth factor receptor-bound protein 2 \\
\hline 3.65432 & 65 & PLCG1 & P19174-2 & 1-phosphatidylinositol 4.5-bisphosphate phosphodiesterase gamma-1 \\
\hline 3.5852 & 17 & GRAP & Q13588 & GRB2-related adapter protein \\
\hline 3.498 & 37 & CD2AP & Q9Y5K6 & $\mathrm{CD} 2$-associated protein \\
\hline 3.44042 & 24 & CBLB & Q13191 & E3 ubiquitin-protein ligase $C B L-B$ \\
\hline 3.26881 & 48 & LRRK1 & Q38SD2 & Leucine-rich repeat serine/threonine-protein kinase 1 \\
\hline 3.09667 & 2 & SHQ1 & Q6PI26 & Protein SHQ1 homolog \\
\hline 3.04429 & 5 & KNTC1 & P50748 & Kinetochore-associated protein 1 \\
\hline 2.83094 & 4 & socs1 & 015524 & Suppressor of cytokine signaling 1 \\
\hline 2.75931 & 58 & sos2 & Q07890 & Son of sevenless homolog 2 \\
\hline 2.36972 & 34 & PIK3R1 & P27986 & Phosphatidylinositol 3-kinase regulatory subunit alpha \\
\hline 2.30457 & 5 & GAB1 & Q13480 & GRB2-associated-binding protein 1 \\
\hline 2.27569 & 46 & ARAP1 & Q96P48 & Arf-GAP with Rho-GAP domain. ANK repeat and PH domain-containing protein 1 \\
\hline 2.07953 & 5 & MTA1 & Q13330 & Metastasis-associated protein MTA1 \\
\hline 2.07413 & 3 & EML2 & 095834 & Echinoderm microtubule-associated protein-like 2 \\
\hline 2.06516 & 3 & SUPT6H & Q7KZ85 & Transcription elongation factor SPT6 \\
\hline 2.02751 & 68 & PLCG2 & P16885 & 1-phosphatidylinositol 4.5-bisphosphate phosphodiesterase gamma-2 \\
\hline 2.00931 & 26 & PTPRC & P08575 & Receptor-type tyrosine-protein phosphatase C \\
\hline
\end{tabular}

Figure 9: Mass spectrometric analysis of the phosphorylated mlgE-ITT interactome.

(A) Amino acid sequence of the $\mathrm{N}$-terminally biotinylated human mlgE phospho-ITT peptide used. (B) Experimental outline for affinity purification of phospho-ITT binding partners from DG75 cells. Lysates from cells labeled with heavy $(H)$ amino acids Lysine $D_{4}($ Lys +4$)$, Arginine ${ }^{13} \mathrm{C}_{6}(\mathrm{Arg}+6)$ were treated with the phospho-ITT peptide and lysates from cells labeled with light (L) amino acids Lysine (Lys+O) and Arginine $(A r g+0)$ were treated with the non-phosphorylated ITT peptide and subsequently purified using streptavidin beads. (C) Scatter plot representing distribution of significantly enriched proteins by plotting normalized ratio of $\mathrm{H} / \mathrm{L}$ vs. intensity. Proteins with a normalized $\mathrm{H} / \mathrm{L}$ ratio of greater than 2.0 fold are highlighted in red with their gene names. (D) Proteins highlighted in (C) are represented in tabular format with values for normalized $\mathrm{H} / \mathrm{L}$ ratio, number of peptides, gene name, protein ID and protein name. Grb2 and Grap are highlighted in red. The mass spectrometry analysis and preliminary data processing was done in collaboration with the Proteomics Core Facility of University Medicine Göttingen. Data are from one experiment. 
A

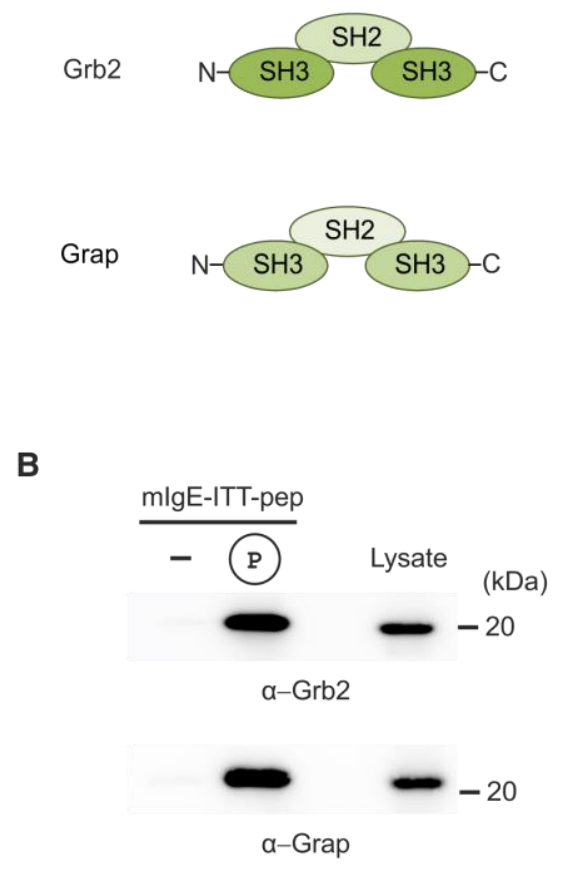

C

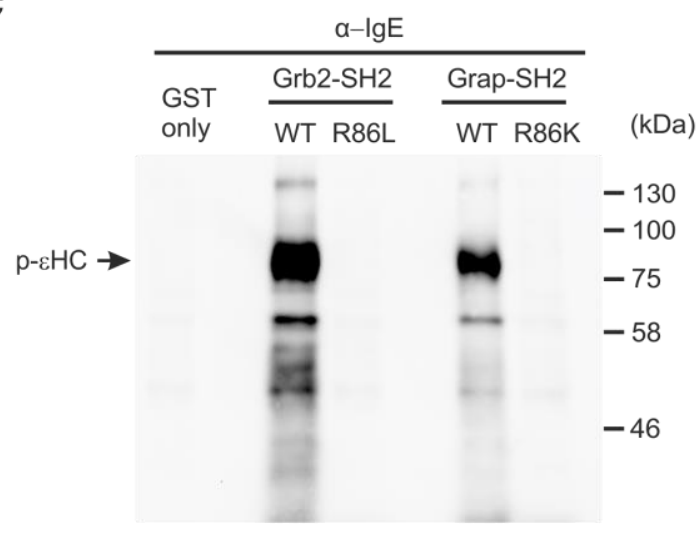

$a-p-T y r 100$

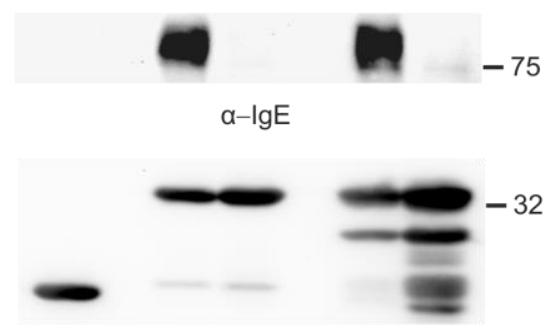

a-GST

Figure 10: The mlgE-ITT binds to the closely related adaptor proteins Grb2 and Grap.

(A) Illustration depicting the domain architecture of Grb2 and Grap. (B) Cell lysates from wild-type DG75 cells were used to purify binding partners using the biotinylated mlgE phospho-ITT peptide (sequence in Fig. 9A) and streptavidin beads. The non-phosphorylated peptide was used as negative control and the purified samples were analyzed by SDS-PAGE and immunoblotting by probing for Grb2 and Grap. (C) DG75 cells expressing a WT mlgE-BCR were stimulated for $3 \mathrm{~min}$ with $10 \mu \mathrm{g} / \mathrm{ml}$ anti-human-lgE antibody and cell lysates were prepared. The lysates were mixed with $15 \mu \mathrm{g}$ GST-tagged functional Grb2 and Grap SH2 domains (WT), SH2 domain mutants (R86L for Grb2 and R86K for Grap) or GST only, along with glutathione beads to purify interaction partners. Binding partners were analyzed by SDS-PAGE and immunoblotting. The phosphorylated mlgE-ITT was detected using the $p$-Tyr antibody. The blot was also probed with anti-IgE and anti-GST antibodies. Data are representative of three independent experiments.

In order to validate the mass spectrometry results, I biochemically tested for binding of Grap to the mlgE-ITT motif by two complementary approaches. First, I used the same synthetic peptide as described above to purify binding partners from cell lysates and confirmed the interaction with Grb2 and Grap by immunoblotting (Fig. 10B). Second, I used the SH2 domains of Grb2 and Grap as GST fusion proteins to purify the phosphorylated mlgE-BCR. Inactivated variants of the SH2 domains (R86L and R86K respectively) and GST served as negative controls. I could specifically detect the phosphorylated $\mathrm{\varepsilon m}$ heavy chain in the purified fraction obtained from the WT SH2 domains of Grb2 and Grap (Fig. 10C). Thus, both Grb2 and Grap can directly bind to the phosphorylated mlgE-ITT via their SH2 domains. 


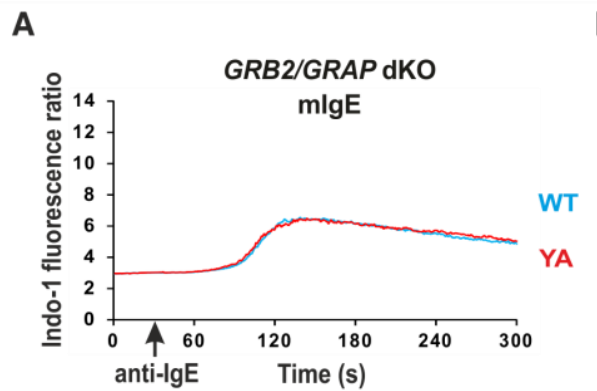

B

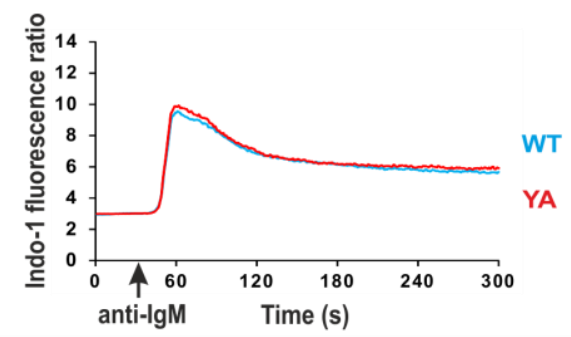

C

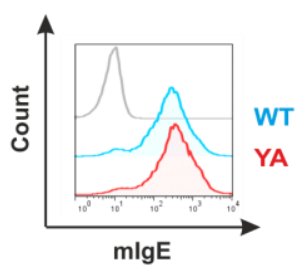

Figure 11: mlgE-ITT-mediated signal amplification is lost in the absence of Grb2 and Grap.

DG75 GRB2/GRAP dKO cells that were transfected with WT and ITT-YA mlgE-BCRs. (A) $\mathrm{Ca}^{2+}$ mobilization kinetics of the cells upon stimulation with $10 \mu \mathrm{g} / \mathrm{ml}$ anti-human-lgE antibody. (B) The endogenous mlgM-BCR induced $\mathrm{Ca}^{2+}$ flux of the cells using $20 \mu \mathrm{g} / \mathrm{ml}$ anti-human-IgM F(ab)' ${ }_{2}$ fragments. (C) Surface expression of the mlgE-BCR variants in these cells. Data are representative of four independent experiments.

To test the functional significance of Grap binding to the mlgE-ITT, I used GRB2/GRAP double-deficient DG75 cells (referred to as GRB2/GRAP dKO) (provided by Caren Bartsch) and expressed the WT and ITT-YA mlgE-BCRs in these cells (Fig. 11C). Indeed, ITT-mediated signal amplification was completely abrogated in the absence of both the adaptor molecules (Fig. 11A). mlgM-BCR signaling was similar for cells with either ITT variant (Fig. 11B). Thus, unlike the mlgG-ITT, the mlgE-ITT utilizes both Grb2 and Grap for BCR-mediated signal amplification in human B cells.

In order to eliminate the possibility of clonal selection-induced side effects causing the ITT phenotype in the DG75 GRB2/GRAP dKO, I reconstituted expression of the adaptor proteins in these cells. Retroviral constructs encoding GRB2-IRES-GFP and GRAP-IRESRFP were used for the reconstitution (schematic depiction in Fig. 12A). Since the adaptor proteins are quite small themselves (about $25 \mathrm{kDa}$ ), the fluorescent markers were not directly coupled to the proteins but expressed separately using an IRES site. The fluorescent markers enable selection of a pure positive population of cells via cell sorting. The GRB2/GRAP dKO cells expressing WT or ITT-YA mlgE-BCRs expressed similar amounts of GFP and or RFP post-sorting (Fig. 12B and C respectively). To directly test the expression of Grb2 and Grap in the sorted cells, I performed immunoblotting and confirmed that the adaptor proteins were expressed at comparable levels (Fig. 12D). Next, I examined the $\mathrm{Ca}^{2+}$ signaling properties of the reconstituted cells and used DG75 GRB2/GRAP dKO and wild-type cells as controls. Again, the WT mlgE-BCR showed enhanced $\mathrm{Ca}^{2+}$ mobilization as compared to the ITT-YA mutant in wild-type cells (Fig. $13 \mathrm{~A}$ ) and lost this capability in GRB2/GRAP dKO cells (Fig. 13B). Reconstitution with Grb2 (Fig. 13C), Grap (Fig. 13D) or both Grb2+Grap (Fig. 13E), all restored ITT mediated signal amplification in the GRB2/GRAP dKO background. There is also a certain degree of co-operativity in the mode of action of Grb2 and Grap, since the cells reconstituted with both adaptors showed higher signal amplification than the individually reconstituted cells. mlgM-BCR induced signaling was the same for cells expressing either mlgE-BCR variant, confirming equivalent internal signaling capabilities (Fig. 13F-J). 
A

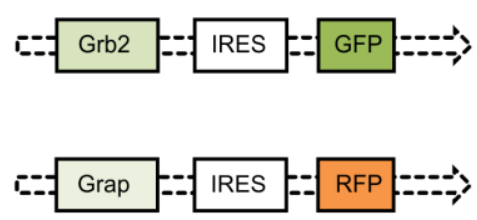

B
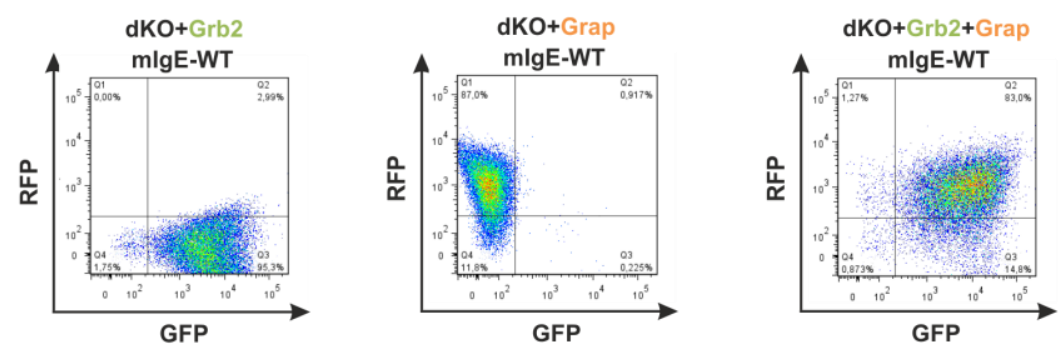

C
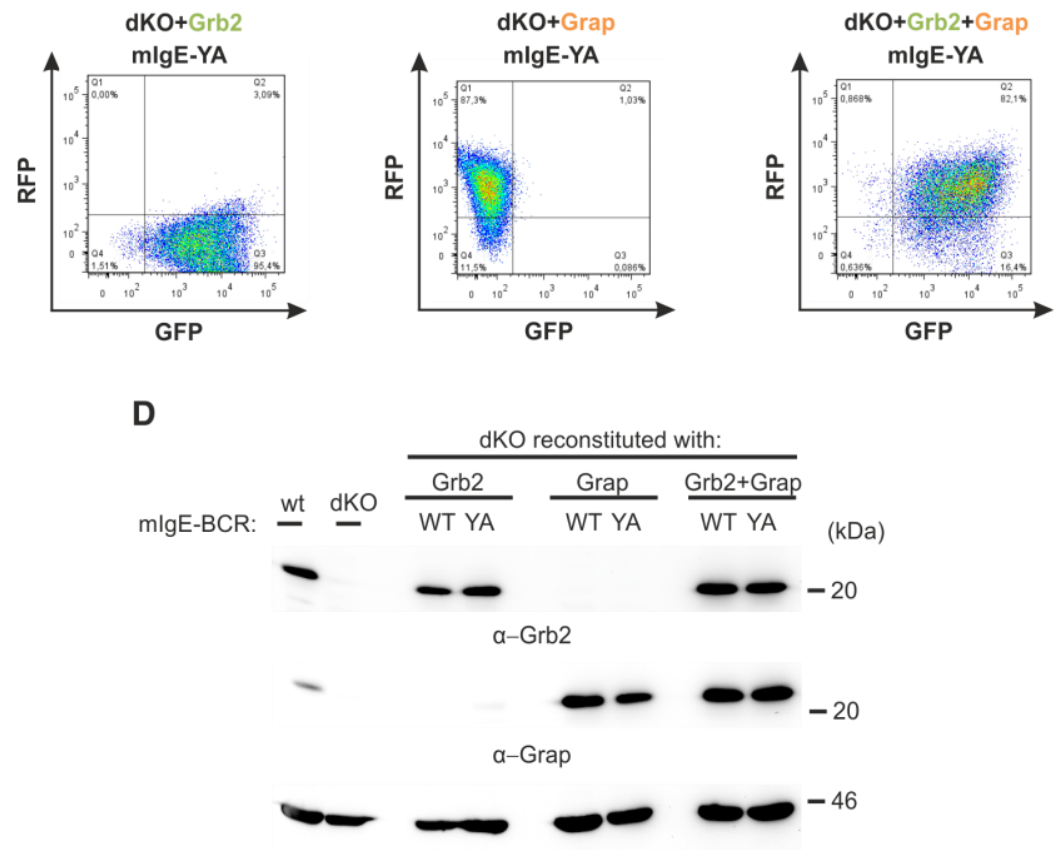

$\alpha-\beta$ actin

Figure 12: Reconstitution of the DG75 GRB2/GRAP dKO with the adaptor proteins Grb2 and Grap.

(A) Schematic illustration of the retroviral expression vectors used for reconstitution of the DG75 GRB2/GRAP dKO cells (dKO) with either GRB2-IRES-GFP or GRAP-IRES-RFP or both. Flow cytometric analysis of dKO cells expressing either WT (B) or ITT-YA (C) mlgE-BCRs that were sorted for GFP-positive (dKO+Grb2), RFP-positive (dKO+Grap) or GFP/RFP double-positive cells (dKO+Grb2+Grap). Cell lysates from the sorted cells were analyzed by SDS-PAGE and immunoblotting for expression of Grb2 and Grap (D). $\beta$-actin levels were analyzed to confirm equal loading. Data are representative of three $(B, C)$ or one (D) independent experiments.

Intriguingly, analysis of the signal strength of the mlgM-BCR in GRB2/GRAP dKO cells, showed reduction in $\mathrm{Ca}^{2+}$ mobilization compared to wild-type DG75 cells (compare Fig. $13 F$ and $G$ ). This phenotype could also be restored by reconstitution with either or both adaptor proteins (Fig. 13H-J). Lastly, the surface expression of the WT and ITT-YA mlgEBCRs was comparable in all cells analyzed (Fig. 13K-O). Thus, both Grb2 and Grap not only amplify signaling at the mlgE-BCR via the ITT motif, but also contribute to canonical mlgM-BCR signaling, which is initiated exclusively via the ITAM motifs in $\lg \alpha-\lg \beta$. 
Results
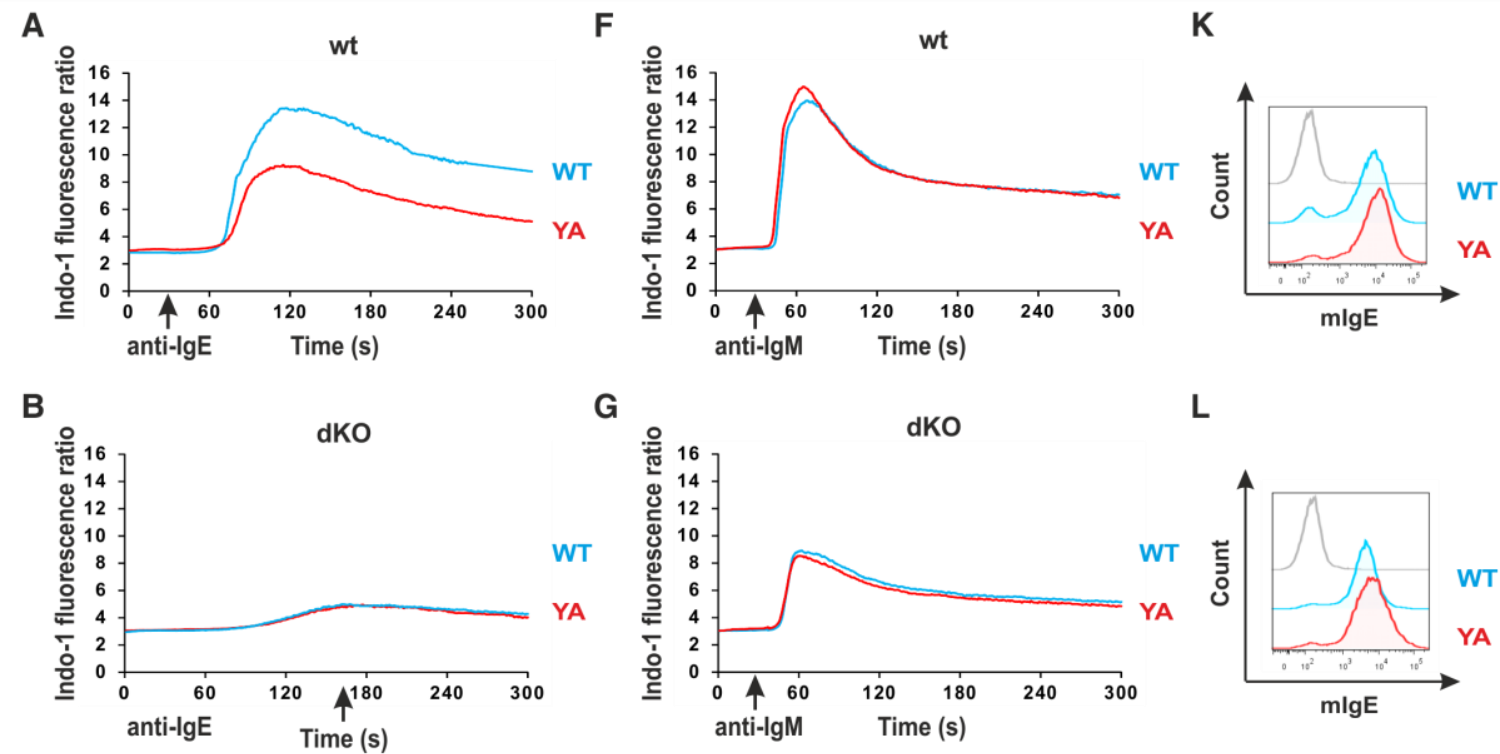

G

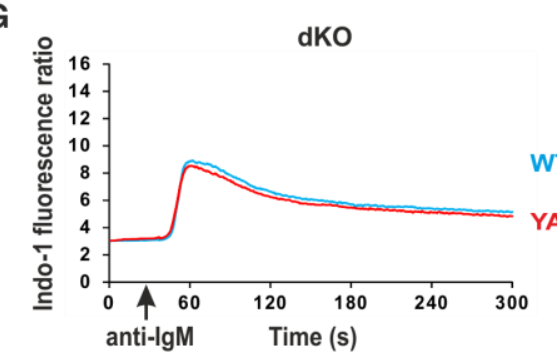

$\mathbf{L}$

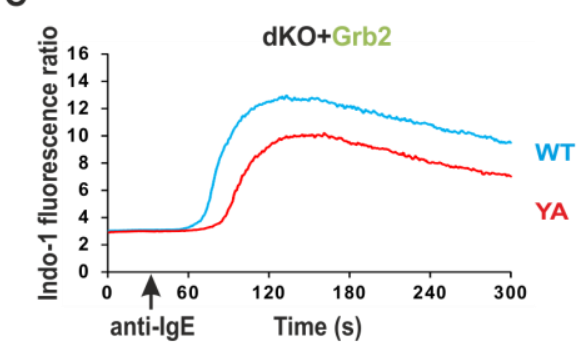

$\mathbf{H}$
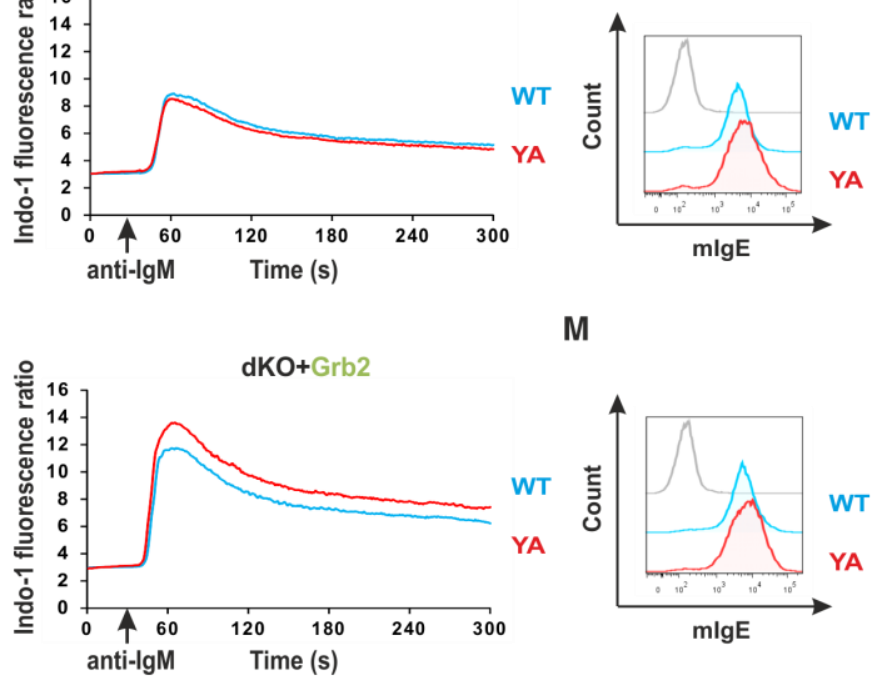

M

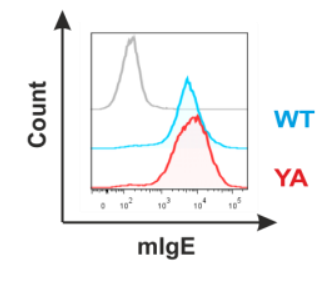

D

I
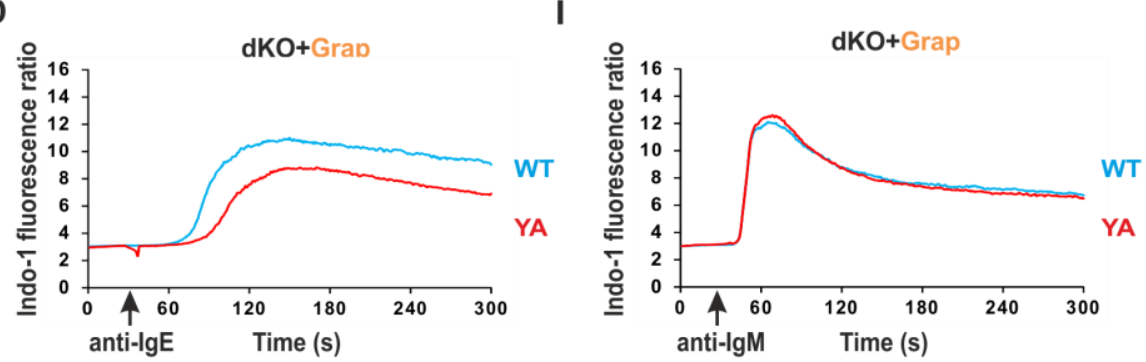

N

E

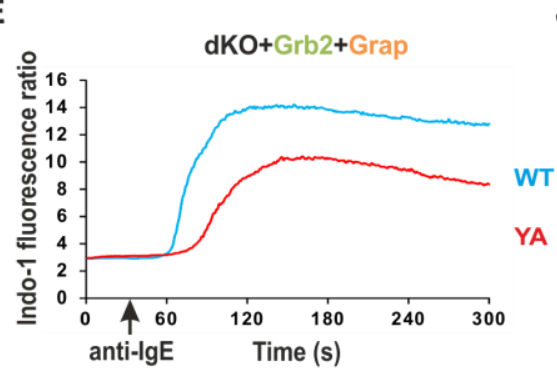

J
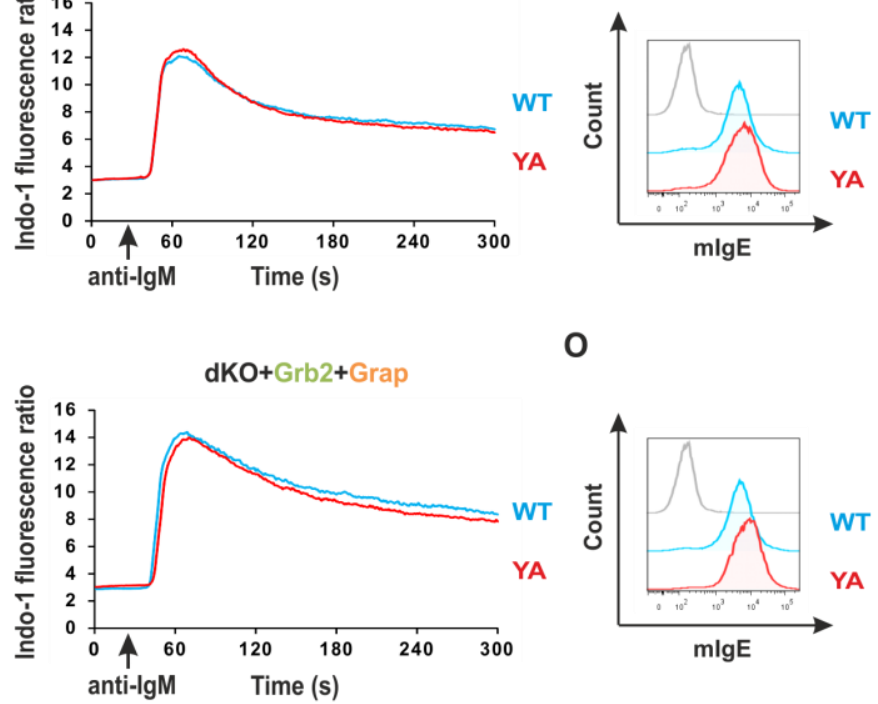

0

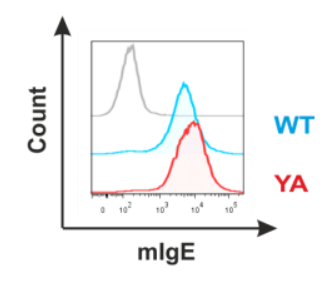

Figure 13: Both Grb2 and Grap contribute to mlgE-ITT-mediated signal amplification.

$\mathrm{Ca}^{2+}$ mobilization kinetics of DG75 cells expressing WT or ITT-YA mutant mlgE-BCR following stimulation with $10 \mu \mathrm{g} / \mathrm{ml}$ anti-human-lgE antibody (A-E) or $20 \mu \mathrm{g} / \mathrm{ml}$ anti-human-IgM F(ab)' 2 fragments (F-J) in the following cellular backgrounds: wild-type (A,F), GRB2/GRAP dKO (B,G), GRB2/GRAP dKO reconstituted with Grb2 $(\mathbf{C}, \mathrm{H})$, GRB2/GRAP dKO reconstituted with Grap (D,I), GRB2/GRAP dKO reconstituted with Grb2+Grap (E,J) respectively. Surface mlgE expression of the ITT variants in DG75 wild-type (K), GRB2/GRAP dKO (L), GRB2/GRAP dKO reconstituted with Grb2 (M), GRB2/GRAP dKO reconstituted with Grap (N), GRB2/GRAP dKO reconstituted with Grb2+Grap (0). Data are representative of three independent experiments. 
A

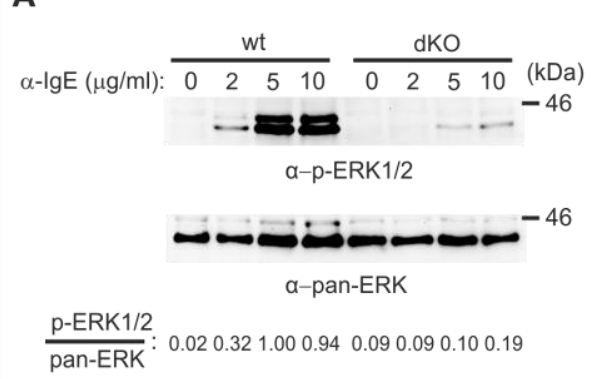

B

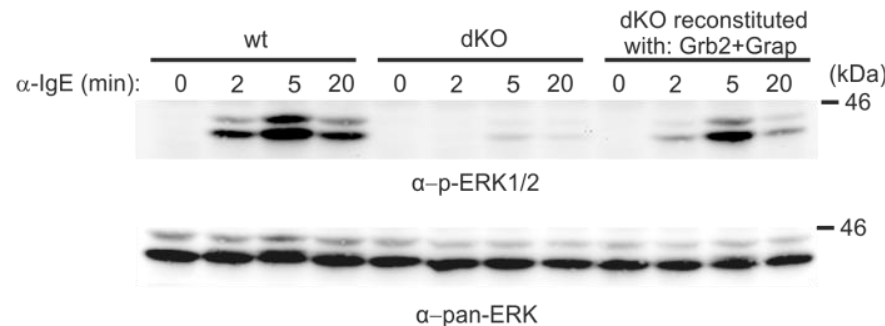

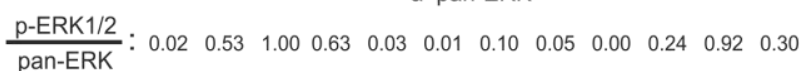

C

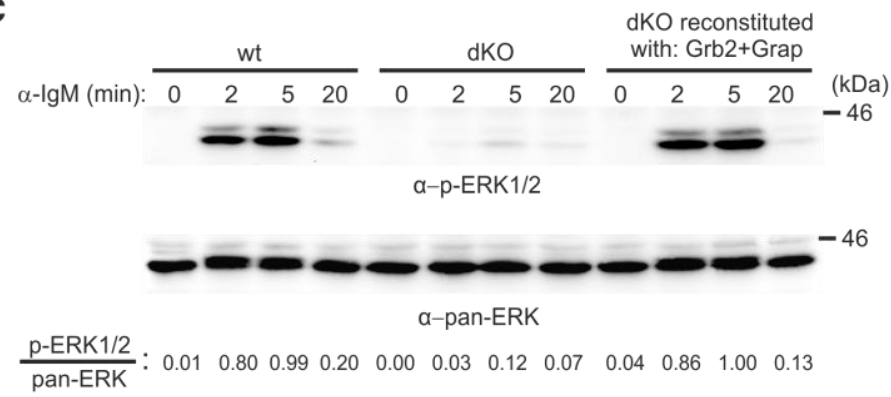

Figure 14: mlgE- and mlgM-BCR induced ERK activation is dependent on Grb2 and Grap.

(A) Cell lysates were prepared from wild-type and GRB2/GRAP dKO DG75 cells following stimulation with the indicated concentrations of anti-human-lgE antibody for $5 \mathrm{~min}$. ERK phosphorylation was analyzed using SDS-PAGE and immunoblotting with antibodies against phospho-ERK (p-ERK) and total ERK (pan-ERK). Relative levels of phospho-ERK were quantified and are indicated below. (B and C) Wild-type DG75 cells, GRB2/GRAP dKO cells and GRB2/GRAP dKO cells reconstituted with both Grb2 and Grap, and expressing a WT mlgE-BCR were stimulated with $10 \mu \mathrm{g} / \mathrm{ml}$ anti-human-lgE antibody (B) or $20 \mu \mathrm{g} / \mathrm{ml}$ anti-human-lgM $\mathrm{F}(\mathrm{ab}){ }^{\prime} 2$ fragments $(\mathbf{C})$. The cell lysates were then analyzed by SDS-PAGE and immunoblotting for ERK activation by detecting phosphorylated ERK (p-ERK) and total ERK (pan-ERK). Relative levels of phosphorylated ERK were quantified and are given below each panel. Data are representative of three independent experiments.

As an alternative signaling readout, I tested mlgE-BCR-induced ERK phosphorylation in the GRB2/GRAP dKO cells at various concentrations of stimulating antibody. Compared to wild-type cells, ERK phosphorylation is strongly diminished in the absence of Grb2 and Grap (Fig. 14A). This phenotype may be attributed to the interaction of Grb2 and Grap with the GEF, Son of sevenless (Sos). Sos is known to induce ERK activation via the RasMAPK pathway following mlgM-BCR stimulation (Kumar et al 1995). mlgE-BCR-mediated ERK phosphorylation is indeed restored upon reconstitution of the GRB2/GRAP dKO cells with both Grb2 and Grap (Fig. 14B). As control, I also tested for mlgM-BCR-induced ERK phosphorylation in the same cells and confirmed the need for Grb2 and Grap for efficient ERK activation by this BCR isotype (Fig. 14C).

Combining all these data, I discovered that upon activation of the mlgE-BCR, the ITT is phosphorylated by Syk leading to binding of Grb2 and/or Grap to the phosphorylated ITT. This leads to enhanced $\mathrm{Ca}^{2+}$ mobilization and efficient ERK phosphorylation via ITTcontaining mlgE-BCRs. Furthermore, an unexpected finding was the role of Grb2 and Grap in positively regulating mlgM-BCR induced $\mathrm{Ca}^{2+}$ signaling as well. Thus, Grb2 and Grap, positively regulate mlgE- and mlgM-BCR induced signaling processes in human B cells. 
Results

\subsection{Deficiency of Grb2 and Grap prevents optimal activation of the canonical ITAM signaling pathway.}

The reduced mlgM-BCR-induced $\mathrm{Ca}^{2+}$ flux in the absence of Grb2 and Grap was a surprising finding since these proteins have never been implied to play a role in positively regulating the early events of mlgM-BCR activation. Therefore, I compared the $\mathrm{Ca}^{2+}$ mobilization kinetics of the mlgE-BCR and the mlgM-BCR in wild-type, GRB2 KO and GRB2/GRAP dKO cells. The effect of the absence of the adaptor proteins seems to be more profound on mlgE-induced signaling, probably due to the combined impact on ITAM and ITT signaling (Fig. 15A). The reduction on mlgM-BCR signaling is comparatively moderate but still apparent (Fig. 15B). The diminished BCR-induced signaling was not due to reduced BCR expression, which was comparable in all cellular backgrounds (mlgE expression in Fig. 15C and data not shown).

Given that $\mathrm{BCR}$-induced $\mathrm{Ca}^{2+}$ mobilization is controlled by a set of signaling proteins, collectively termed the $\mathrm{Ca}^{2+}$ initiation complex, I decided to analyze how Grb2 and Grap affect this complex. The tyrosine kinase Syk, the adaptor protein SLP65 and the phospholipase PLC $\gamma 2$ were examined. To test their activation, I used cell lysates from wild-type and GRB2/GRAP dKO DG75 cells stimulated via the mlgE- or mlgM-BCR. Analysis of PLC $\gamma 2$ phosphorylation following affinity purification with an anti-PLC $\gamma 2$ antibody revealed that PLC $\gamma 2$ activation was markedly reduced in the absence of Grb2 and Grap (Fig. 15D). Again the effect was more pronounced for the mlgE-BCR than the mlgM-BCR. I also used the same lysates and tested for phosphorylation of SLP65 (Fig. 15E upper panel) and Syk (Fig. 15E lower panel). Both of these proteins also exhibited reduced activation in the absence of Grb2 and Grap. Thus, Grb2 and Grap somehow act upstream in the BCR signaling cascade by promoting efficient phosphorylation and activation of Syk, SLP65 and PLC $\gamma 2$, downstream of both mlgM- and particularly mlgEBCRs.

Both Grb2 and Grap have a central SH2 domain that binds phospho-tyrosine residues within the $\mathrm{YxN}$ core motif which recruits the proteins to signaling complexes. The $\mathrm{SH} 2$ domain of either protein is flanked by two $\mathrm{SH} 3$ domains that constitutively bind effector proteins that relay the signal further. To determine which domains are essential for the function of Grb2, I reconstituted GRB2/GRAP dKO cells with various mutant versions of full-length Grb2 using the IRES-GFP vector to enable sorting of GFP-positive cells following transfection. The wild-type Grb2 (WT) or inactivated variants of the N-terminal SH3 domain, central SH2 domain or C-terminal SH3 domain (W36K, R86K and W193K respectively) were used (Fig. 16A). Upon stimulation of both the mlgE-BCR and the mlgM-BCR, the SH2 domain mutant could not restore the signaling defect of GRB2/GRAP dKO cells (Fig. 16B,C blue curve). However, the role of the two SH3 domains of Grb2 differs depending on the mlg isotype analyzed. The mlgE-BCR seems to utilize either of the two Grb2 SH3 domains since both, the W36K and W193K mutant could independently 


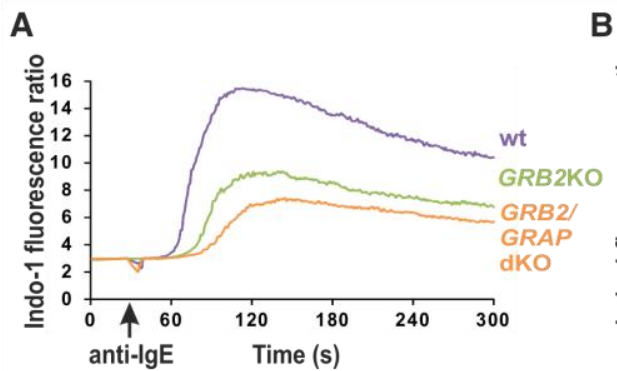

D

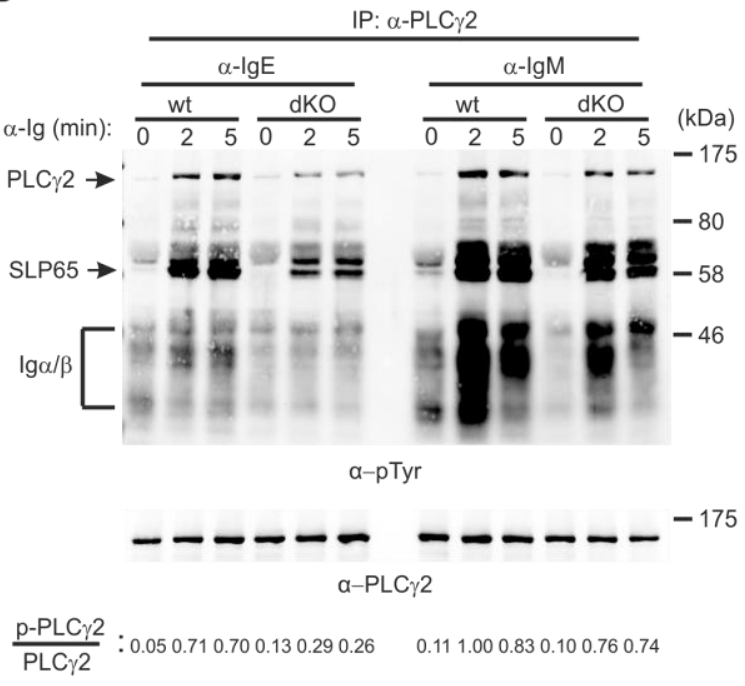

C

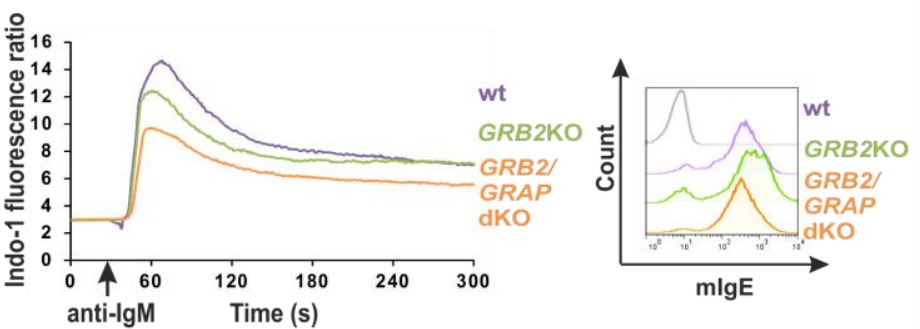

E

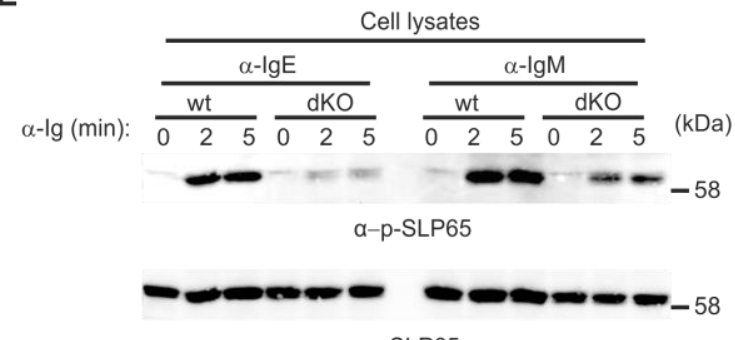

a-SLP65

$\frac{\text { p-SLP65 }}{\text { SLP65 }}: 0.040 .700 .680 .150 .290 .34 \quad 0.270 .971 .000 .330 .780 .64$
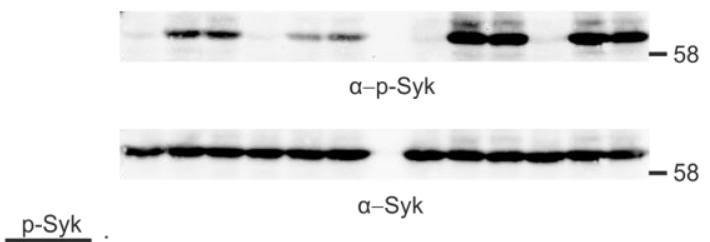

$\frac{\text { p-Syk }}{\text { Syk }}: 0.090 .490 .510 .040 .210 .32 \quad 0.111 .000 .930 .110 .910 .91$

Figure 15: The absence of Grb2 and Grap reduces activation of the $\mathrm{Ca}^{2+}$ initiation complex proteins.

$\mathrm{Ca}^{2+}$ flux of wild-type DG75 cells, GRB2 KO cells and GRB2/GRAP dKO cells expressing WT mlgE-BCRs upon stimulation with $10 \mu \mathrm{g} / \mathrm{ml}$ anti-human-IgE antibody $(A)$, or $20 \mu \mathrm{g} / \mathrm{ml}$ anti-human-lgM $\mathrm{F}(\mathrm{ab})_{2}$ fragments (B). (C) Surface expression of the mlgE-BCR in the different DG75 cell variants. (D) Wild-type DG75 cells and GRB2/GRAP dKO cells expressing WT mlgE-BCRs were left unstimulated (0) or stimulated for the indicated times with $10 \mu \mathrm{g} / \mathrm{ml}$ anti-human-lgE antibody or $20 \mu \mathrm{g} / \mathrm{ml}$ anti-human-IgM $\mathrm{F}(\mathrm{ab}){ }_{2}$ fragments. Cell lysates were mixed with $2 \mu \mathrm{g}$ anti-PLC $\gamma 2$ antibody and protein AG sepharose beads to purify PLC $\gamma 2$. The purified proteins were analyzed by SDS-PAGE and immunoblotting with anti-p-Tyr100 antibody to measure activation of PLC $\gamma 2$. The anti-PLC $\gamma 2$ antibody was used as loading control and the ratio of phospho-PLC $\gamma 2$ was quantified and is indicated at the bottom. (E) Activation of SLP65 and Syk was analyzed by using the cell lysates from (D) and probing with anti-p-SLP65 (upper panel) and anti-p-Syk (lower panel) antibodies. The total SLP65 and total Syk antibodies were used to quantify the ratio of phospho-SLP65 and phospho-Syk and is indicated below each panel. Data are representative of three independent experiments.

restore $\mathrm{Ca}^{2+}$ flux to wild-type levels (Fig. 16B). In contrast, the $\mathrm{N}$-terminal SH3 domain is required for mlgM-BCR induced $\mathrm{Ca}^{2+}$ flux and the $\mathrm{C}$-terminal $\mathrm{SH} 3$ domain is dispensable for that process (Fig. 16C). GFP expression was similar for all transfectants (Fig. 16D). Again, as an additional BCR signaling readout, I analyzed ERK phosphorylation in these cells (Fig. 16E). All three Grb2 domains are required for ERK activation as only the wildtype Grb2 protein could restore mlgE-BCR-induced ERK phosphorylation in the GRB2/GRAP dKO background. 
A

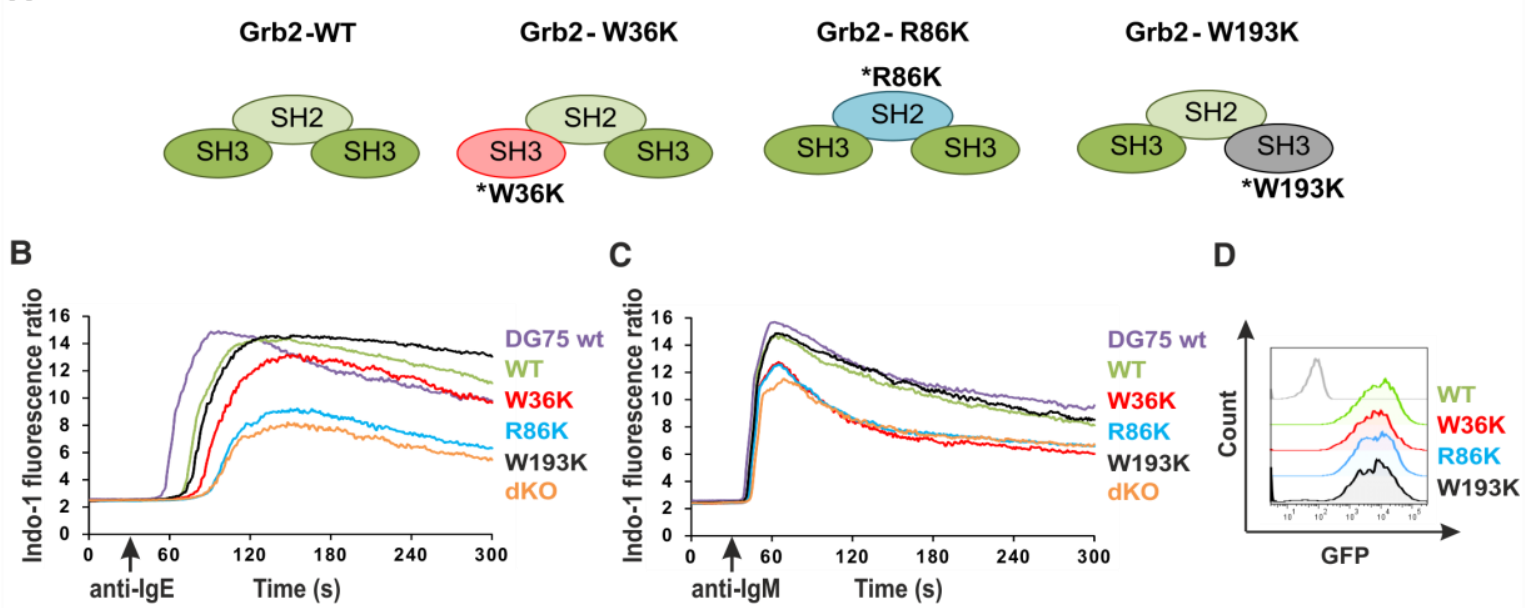

E

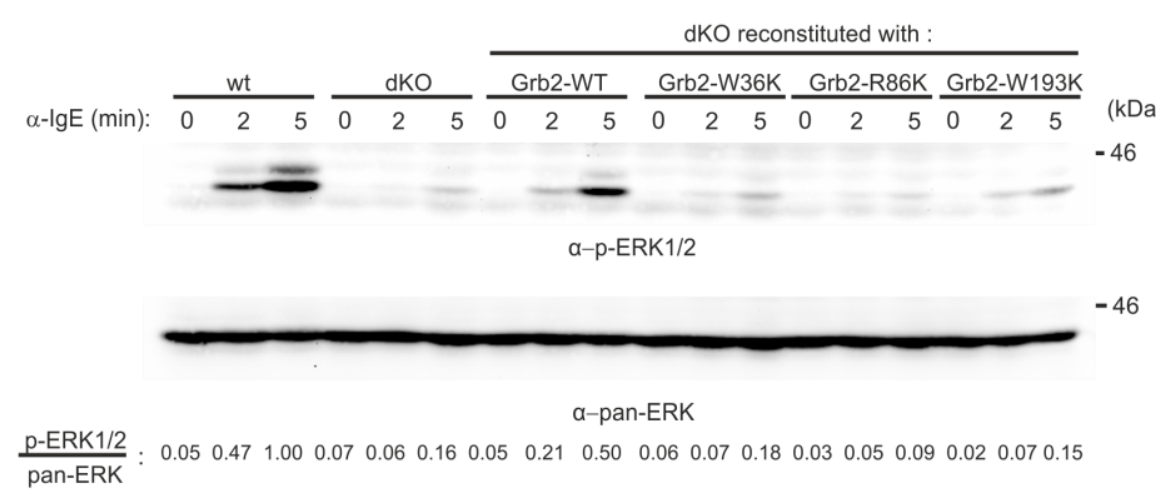

Figure 16: The $\mathrm{N}$ - and C-terminal SH3 domains of Grb2 have different roles in mlgE- and mlgM-BCR signaling.

(A) Schematic representation of the Grb2 variants used: Grb2 wild-type (WT), N-terminal SH3 domain mutant (W36K), SH2 domain mutant (R86K) and C-terminal SH3 domain mutant (W193K). (B-E) DG75 GRB2/GRAP dKO cells expressing the WT mlgE-BCR were reconstituted with Grb2 variants using the IRES-GFP vector and GFP-positive cells were sorted for further analysis. Wild-type (wt) and GRB2/GRAP dKO (dKO) cells expressing the WT mlgE-BCR served as controls. $\mathrm{Ca}^{2+}$ mobilization by the cells upon stimulation with 10 $\mu \mathrm{g} / \mathrm{ml}$ anti-human-lgE antibody (B) or $20 \mu \mathrm{g} / \mathrm{ml}$ anti-human-IgM F(ab)' 2 fragments (C). (D) GFP expression in the reconstituted cells. (E) The same cells as above were stimulated with $10 \mu \mathrm{g} / \mathrm{ml}$ anti-human-lgE antibody for 2 or 5 min or left unstimulated (0). Cell lysates were prepared and ERK phosphorylation was analyzed by SDS-PAGE and immunoblotting with antibodies against phospho-ERK (p-ERK) or total ERK (pan-ERK). Relative levels of phospho-ERK to total ERK were quantified and are indicated below. Data are representative of three independent experiments.

Grb2 and Grap share about $60 \%$ homology in amino acid sequence. It is published that the Grb2 N-terminal SH3 domain binds the PLC $\gamma 2$-activating kinase Btk, whereas the Grap N-terminal SH3 domain cannot (Engels et al 2014). Hence, there do exist differences in the functional capabilities of these closely related adaptor proteins. Accordingly, I also examined the functional properties of the Grap domains by reconstituting the GRB2/GRAP dKO cells with wild-type Grap (WT) or inactivated Nterminal SH3 domain, $\mathrm{SH} 2$ domain or C-terminal SH3 domain variants (W36K, R86K and W195K respectively) (Fig. 17A). This time, the IRES-RFP vector was used to sort for RFP-positive transfectants. 
A

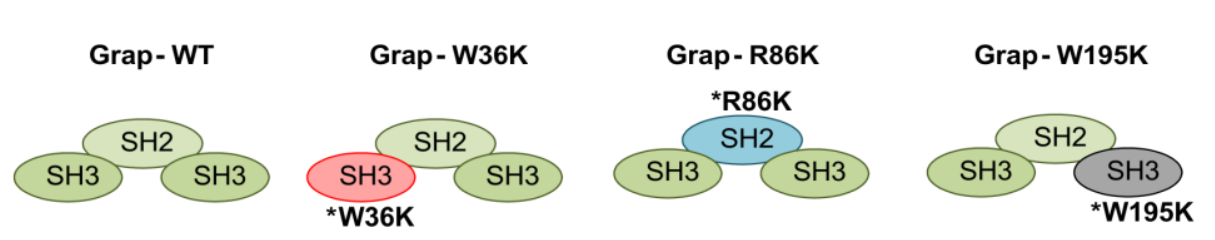

B

C

D
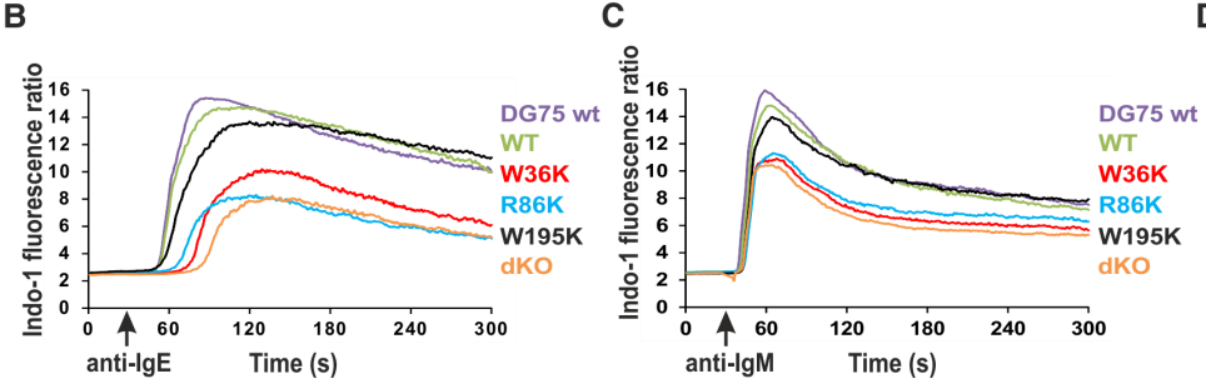

E

dKO reconstitited with:

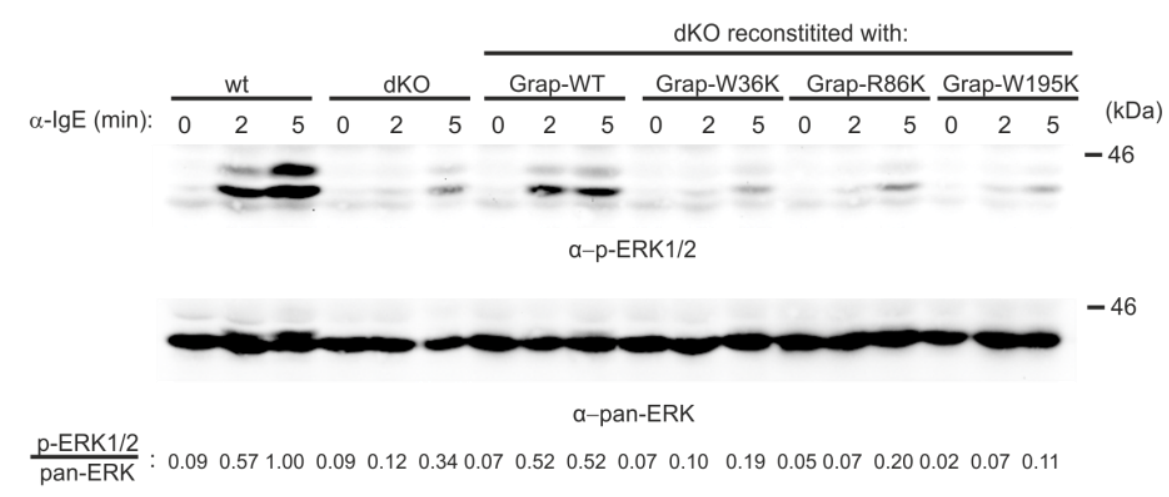

Figure 17: The N-terminal SH3 domain of Grap is required for optimal mlgE- and mlgM-BCR-mediated signaling

(A) Schematic representation of the Grap variants used: Grap wild-type (WT), N-terminal SH3 domain mutant (W36K), SH2 domain mutant (R86K) and C-terminal SH3 domain mutant (W195K). (B-E) DG75 GRB2/GRAP dKO cells expressing the WT mlgE-BCR were reconstituted with Grap variants using the IRES-RFP vector and RFP-positive cells were sorted for further analysis. Wild-type (wt) and GRB2/GRAP dKO (dKO) cells expressing the WT mlgE-BCR served as controls. $\mathrm{Ca}^{2+}$ mobilization by the cells upon stimulation with 10 $\mu \mathrm{g} / \mathrm{ml}$ anti-human-IgE antibody (B) or $20 \mu \mathrm{g} / \mathrm{ml}$ anti-human-IgM F(ab)' 2 fragments (C). (D) RFP expression in the reconstituted cells. (E) The same cells as above were stimulated with $10 \mu \mathrm{g} / \mathrm{ml}$ anti-human-lgE antibody for 2 or 5 min or left unstimulated (0). Cell lysates were prepared and ERK phosphorylation was analyzed by SDS-PAGE and immunoblotting with antibodies against phospho-ERK (p-ERK) or total ERK (pan-ERK). Relative levels of phospho-ERK to total ERK were quantified and are indicated below. Data are representative of three independent experiments.

Again the $\mathrm{SH} 2$ domain mutant could not restore the $\mathrm{Ca}^{2+}$ flux defect of GRB2/GRAP dKO cells (Fig. 17B,C blue curve). However, The $\mathrm{N}$-terminal SH3 domain seems to be required by both mlgE- and mlgM-BCRs since the W36K mutant could not restore the $\mathrm{Ca}^{2+}$ signaling (Fig. 17B,C red curve). The C-terminal SH3 domain of Grap was found to be dispensable for signaling via the mlgM- and mlgE-BCR. RFP expression was similar for all variants (Fig. 17D). Lastly, ERK phosphorylation was again dependent on all three domains of Grap as only wild-type Grap restored phospho-ERK levels in the GRB2/GRAP dKO cells (Fig. 17E). 


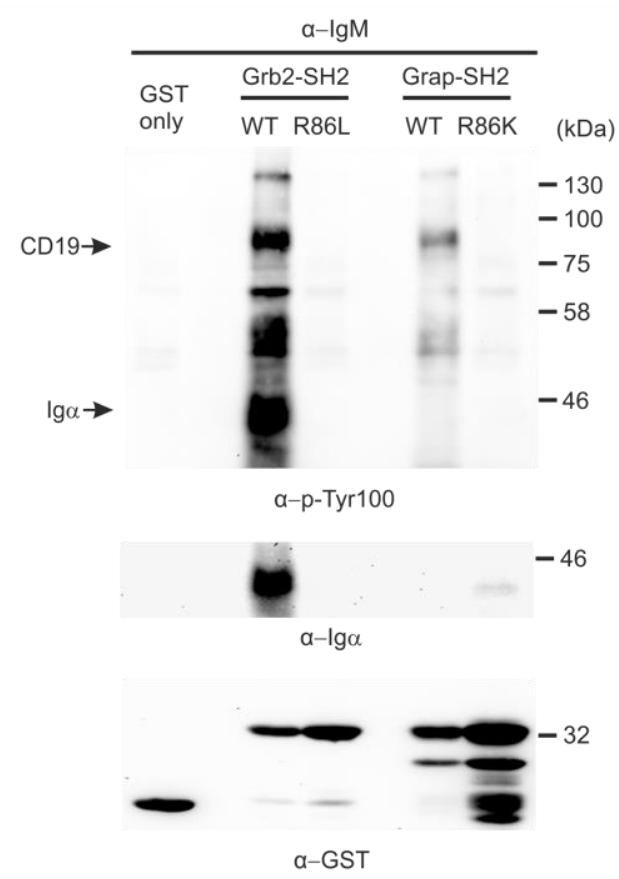

Figure 18: Grb2 and Grap are recruited to the BCR and/or to co-stimulatory molecules upon BCR activation

Wild-type DG75 cells were stimulated for 3 minutes with $20 \mu \mathrm{g} / \mathrm{ml}$ anti-human-IgM $F(a b){ }_{2}$ fragments and cell lysates were prepared. The lysates were mixed with $15 \mu \mathrm{g}$ GST-tagged functional Grb2 and Grap SH2 domains (WT) and SH2 domain mutants (R86L for Grb2 and R86K for Grap) or GST only, along with glutathione beads to purify interaction partners. Purified proteins were analyzed by SDS-PAGE and immunoblotting. Phosphorylated interaction partners of the Grb2/Grap SH2 domains were analyzed using the p-Tyr-100 antibody. The blot was also probed with anti-Ig $\alpha$ and anti-GST antibodies. Data are representative of at least two independent experiments.

Since both Grb2 and Grap require their $\mathrm{SH} 2$ domains for performing their function, I wanted to find the binding partner/s that could recruit these proteins to the active mlgMBCR signalosome. GST fusion proteins containing either the Grb2 or Grap SH2 domain were used in wild-type or inactive configuration (R86L and R86K respectively), along with GST as an additional negative control. The SH2 domains purified a number of tyrosinephosphorylated proteins from mlgM-BCR-stimulated DG75 B cells (Fig. 18 upper panel). I could identify Ig $\alpha$ as a major interaction partner for the Grb2 SH2 domain, an interaction that was lacking in case of the Grap SH2 domain (Fig. 18 second panel). The $90 \mathrm{kDa}$ phospho-band in the anti-phospho-tyrosine blot represents the co-stimulatory molecule CD19, which was bound by both Grb2 and Grap (identified in separate experiments). Although, I cannot rule out other routes of recruitment at this point, I could partially localize the adaptor proteins directly at the BCR or in association with the co-stimulatory molecule CD19. 
Combining these data, I found that upon mIgM-BCR stimulation, a pool of the adaptor proteins is recruited to either Ig $\alpha$ (Grb2) or CD19 (Grb2 and Grap) via their SH2 domains. The presence of Grb2 and Grap at the signalosome contributes to ITAM-induced $\mathrm{Ca}^{2+}$ mobilization via improved activation of the $\mathrm{Ca}^{2+}$ initiation complex proteins, Syk, SLP65 and PLC $\gamma 2$. The effector functions of the SH3 domains of the adaptors differ depending on the $\mathrm{BCR}$ isotype. mlgE-BCR induced $\mathrm{Ca}^{2+}$ flux uses either $\mathrm{Grb} 2 \mathrm{SH} 3$ domain whereas the $\mathrm{N}$-terminal SH3 domain of Grap is critical for this process. On the other hand, mlgM-BCR induced $\mathrm{Ca}^{2+}$ flux selectively utilizes the N-terminal $\mathrm{SH} 3$ domains of Grb2 and Grap for optimal signaling. The exact mechanism behind the function of these domains remains to be determined. Another relevant observation was that optimal ERK phosphorylation requires all three domains of Grb2 and Grap to be functional. Since this is not the case for $\mathrm{Ca}^{2+}$ signaling, the activation of the two pathways by Grb2 and Grap is probably partially independent of each other.

\subsection{Human mlgE can function independently of the $\lg \alpha / \lg \beta$ heterodimer.}

Upon analysis of $\mathrm{Ca}^{2+}$ signaling in wild-type and GRB2/GRAP dKO DG75 cells, I found that the mlgE-BCR is more dependent on the Grb2-family adaptors as compared to the mlgM-BCR. The drop in $\mathrm{Ca}^{2+}$ mobilization in the GRB2/GRAP dKO cells is more significant for the mlgE-BCR even at optimal concentrations of stimulating antibody (Fig. 19A, D). This may be partly explained by the employment of ITT and ITAM signaling by mlgE-BCRs, while mlgM-BCRs use only ITAM signaling. However, the absence of Grb2 and Grap should completely abrogate ITT signaling and thus the WT mlgE-BCR should signal at similar levels as the mlgM-BCR via its ITAMs. Intriguingly, this was not the case. When I titrated down the stimulating antibody to sub-optimal concentrations of $2 \mu \mathrm{g} / \mathrm{ml}$ (Fig. 19B, E) and $1 \mu \mathrm{g} / \mathrm{ml}$ (Fig. 19C, F), I observed that the mlgE-BCR could not signal anymore, whereas the mlgM-BCR still mediated some $\mathrm{Ca}^{2+}$ flux.

Thus, to study the ITAM signaling pathway in both BCR isotypes, I analyzed phosphorylation of Ig $\alpha$ upon BCR stimulation in wild-type DG75 cells (Fig. 20A). mlgMBCR stimulation induced a strong $\lg \alpha$ phosphorylation, whereas stimulation of the WT mlgE-BCR produced a very weak phospho-Ig $\alpha$ signal. The same anti-human-IgE antibody was used earlier and induced very efficient $\mathrm{Ca}^{2+}$ signaling in the very same cells (Fig. 19A). These data would thereby indicate that the mlgE-BCR is mostly using the ITT for signaling with only little ITAM activation. 
A

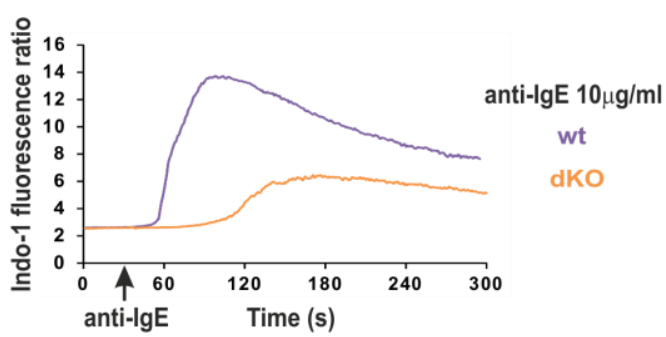

B

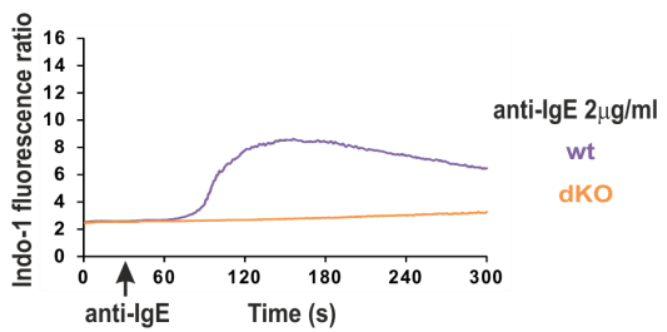

C

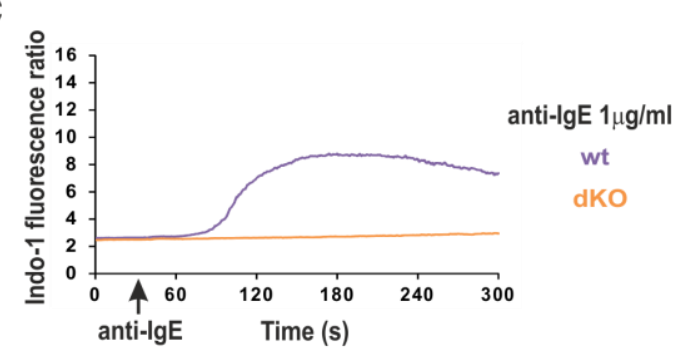

D

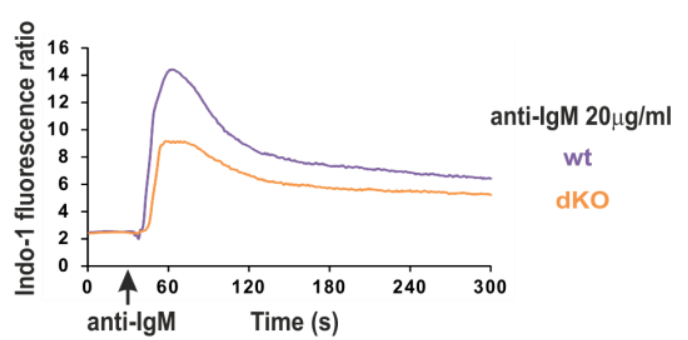

$\mathrm{E}$

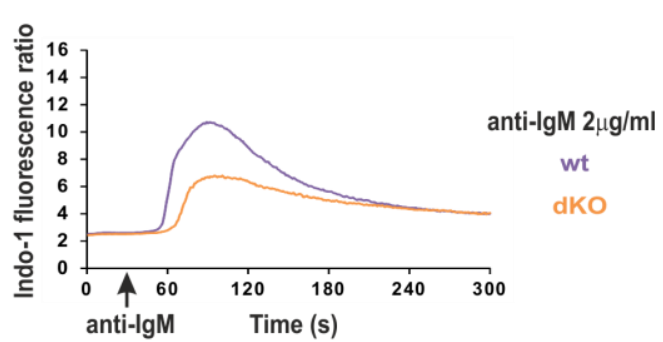

$\mathbf{F}$

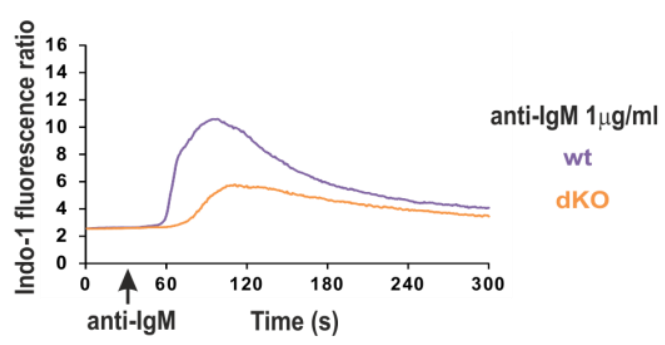

Figure 19: The mlgE-BCR is more dependent on Grb2 and Grap than the mlgM-BCR for signaling in DG75 B cells.

Comparison of $\mathrm{Ca}^{2+}$ mobilization kinetics of wild-type DG75 cells (wt) and GRB2/GRAP dKO cells (dKO) expressing a WT-ITT-containing mlgE-BCR. Cells were stimulated with the indicated concentrations of antihuman-IgE antibody (anti-lgE) (A-C) or anti-human-IgM $F(a b)_{2}$, fragments (anti-lgM) (D-F). Data are representative of three independent experiments.

I wanted to validate the observation of minimal mlgE-BCR-induced $\lg \alpha$ phosphorylation through another complementary approach. Since I had previously found that the Grb2 $\mathrm{SH} 2$ domain efficiently purified phospho-Ig $\alpha$ (Fig. 18), I used this $\mathrm{SH} 2$ domain as an affinity matrix to purify $\lg \alpha$ upon mlgM- and mlgE-BCR stimulation (Fig. 20B). Upon mlgMBCR stimulation of DG75 cells, the Grb2 SH2 domain purified large amounts of phospho$\lg \alpha$, whereas $\mathrm{mlg} E-B C R$ stimulation lead to very little phospho-lg $\alpha$ being purified. The $\lg \mathrm{E}$ stimulation was successful since the ITT got phosphorylated and the phosphorylated mlgE molecule was efficiently purified. Thus, I conclude that there is minimal Ig $\alpha$ phosphorylation by the mlgE-BCR in DG75 cells. As a side note, the Grb2 SH2 domain also purified the co-stimulatory molecule CD19 upon both mlgE- and mlgM-BCR stimulation. This indicates an isotype-independent role of the Grb2-CD19 complex upon BCR stimulation, which would require detailed investigation in the future. 
Results

A

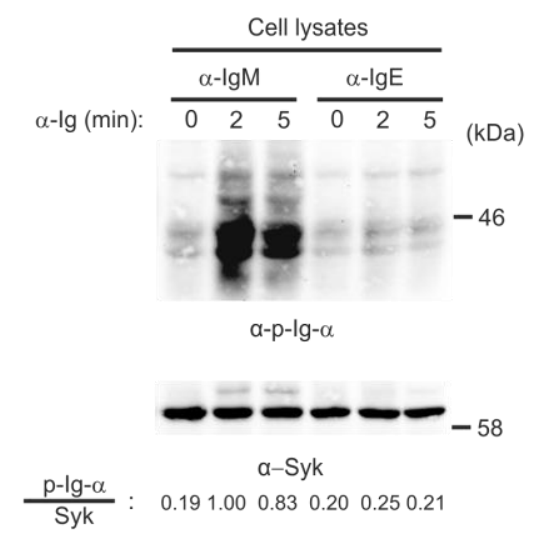

C

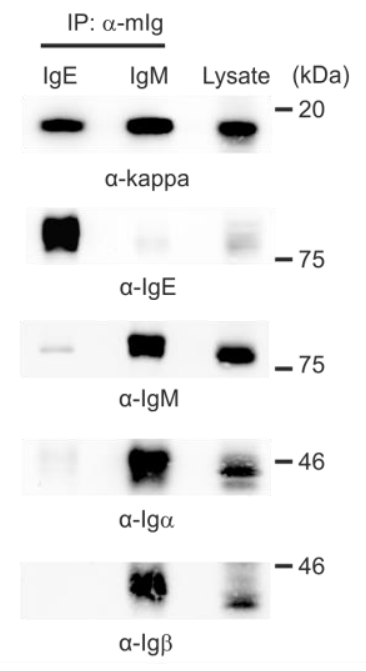

B

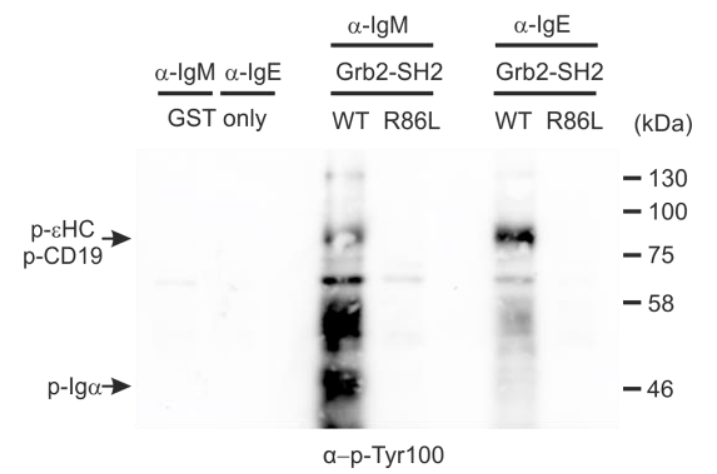

$\alpha-\lg \alpha$
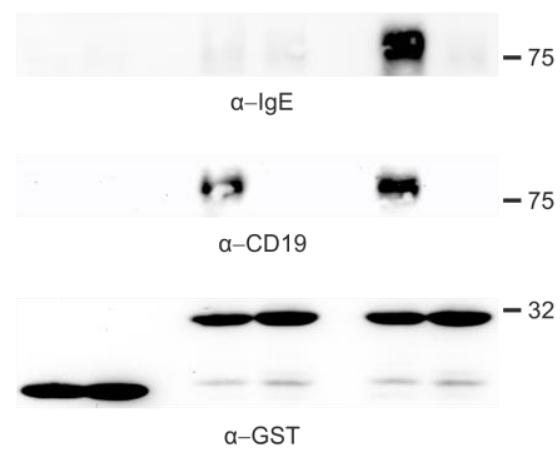

Figure 20: The mlgE-BCR signals despite minimal interaction with $\lg \alpha / \lg \beta$ in DG75 B cells.

(A) Wild-type DG75 cells expressing WT mlgE-BCRs were left unstimulated (0) or stimulated for 2 or 5 min with either $20 \mu \mathrm{g} / \mathrm{ml}$ anti-human-IgM F(ab)'2 fragments ( $\alpha-\operatorname{lgM})$ or $10 \mu \mathrm{g} / \mathrm{ml}$ anti-human-lgE antibody ( $\alpha-\operatorname{lgE})$. Cell lysates were prepared and Ig $\alpha$-ITAM phosphorylation was detected by SDS-PAGE and immunoblotting with an anti-phospho-lg $\alpha$ antibody. Anti-Syk was used as loading control and the quantified ratio of phosphoIg $\alpha$ is indicated below. (B) The same cells as in (A) were stimulated for $3 \mathrm{~min}$ with $20 \mu \mathrm{g} / \mathrm{ml}$ anti-human-lgM $\mathrm{F}(\mathrm{ab})^{\prime}{ }_{2}$ fragments $(\alpha-\operatorname{lgM})$ or $10 \mu \mathrm{g} / \mathrm{ml}$ anti-human-IgE antibody $(\alpha-\operatorname{lgE})$. Cell lysates were mixed with $15 \mu \mathrm{g}$ GST-tagged functional Grb2 SH2 domain (WT), the SH2 domain mutant (R86L) or GST only, along with glutathione beads to purify interaction partners. Purified proteins were analyzed by SDS-PAGE and immunoblotting. Phosphorylated interaction partners of the Grb2 SH2 domain were analyzed by the $p-T y r-100$ antibody. The blot was also probed with antibodies against $\lg \alpha$, IgE, CD19 and GST. (C) The surface pool of mlgE- or mlgM-BCRs was affinity purified by incubating cells for $5 \mathrm{~min}$ on ice with either $10 \mu \mathrm{g} / \mathrm{ml}$ anti-humanIgE-Biotin (IgE) or $10 \mu \mathrm{g} / \mathrm{ml}$ anti-human-IgM-Biotin (IgM) antibodies respectively. Cell lysates were incubated with Streptavidin beads for BCR purification. Interaction with $\lg \alpha, \lg \beta$ was detected using SDS-PAGE and immunoblotting with antibodies against the proteins. Anti-IgM, anti-IgE and anti-kappa light chain antibodies were used to detect the relative levels of purified BCRs. Data are representative of three independent experiments. 
The limited amount of $\lg \alpha$ phosphorylation in the context of the mlgE-BCR raised the question of whether the $\mathrm{mlg} E$ molecule associates with the $\lg \alpha / \lg \beta$ heterodimer. Additionally, the amount of mlgE on the cell surface could be much less than that of the endogenous mlgM-BCR, which could also account for the observed differences in $\lg \alpha$ phosphorylation. To test these theories, I purified the cell surface fraction of mlgM- and mlgE-BCRs from wild-type DG75 cells using biotinylated antibodies against the heavy chains of these proteins (Fig. 20C). To directly compare the amount of purified mlg isotype using the same detection system, I analyzed the levels of the kappa light chain present in the mlgM and mlgE purification samples. I found that the mlgE-associated kappa light chain levels are slightly less but still comparable to the mlgM-bound kappa light chain levels (Fig. 20C top panel). Also, large amounts of $\lg \alpha / \lg \beta$ were co-purified along with $\mathrm{mlgM}$, whereas close to undetectable levels of $\lg \alpha / \lg \beta$ were co-purified with $\mathrm{mlg} E$. To be noted here is the fact that the antibody used for purification of the BCRs is different in both cases (anti-IgM or anti-lgE), which could contribute to differences in purification capabilities of intact BCR complexes. Furthermore, there are some reports that the stability of the $\mathrm{mlg}$ and the $\lg \alpha / \lg \beta$ heterodimer can be different for different $\mathrm{mlg}$ isotypes and is influenced by the detergents used for purification of BCR complexes (Schamel and Reth 2000). Hence, I could not at this stage exclude varied complex stability as a reason behind differences seen in $\lg \alpha / \lg \beta$ association with $\mathrm{mlgM}$ and $\mathrm{mlgE}$.

To overcome the limitations associated with differences in complex stability, I employed a system to analyze cells expressing only mlgM or mlgE on the cell surface. I could then compare surface mlg versus $\lg \alpha$ levels in intact cells without introducing purification related biases. To achieve this, I used another human Burkitt lymphoma cell line called Ramos. Like DG75 cells, Ramos cells also express an endogenous mlgM-BCR which contain a lambda light chain. I used a knockout clone of these cells lacking the Ig heavy chain $(\mathrm{H})$ and the Ig light chain $(\mathrm{L})$ (here on referred to as Ramos $H L \mathrm{KO}$ ) (kindly provided by the lab of Michael Reth, Freiburg). I confirmed the lack of human $\mu$ heavy chain expression in these cells (Fig. 21A upper panel). The cells still express intracellular $\lg \alpha$ and $\lg \beta$ (Fig. 21A) but the proteins do not make it to the cell surface in the absence of mlgM (data not shown). This is an ideal genetic background to introduce either mlgM or $\mathrm{mlg} E$ and analyze the cell surface composition of the BCR complex for cells expressing a single mlg isotype.

I first reconstituted the Ramos $H L$ KO with a mouse lambda-1 lg light chain and subsequently brought in the 4-hydroxy-3-nitrophenylacetyl NP-specific human $\mu \mathrm{m}$ or human $\mathrm{mms}$ Ig heavy chains. NP is a hapten that is commonly used as a model antigen to study antigen-specific B cell responses. The resulting Ramos $H L$ KO cells expressed NPspecific mlgM- or mlgE-BCRs where the epitope binding region is completely identical for both isotypes (Schematically depicted in Fig. 21B). 
A

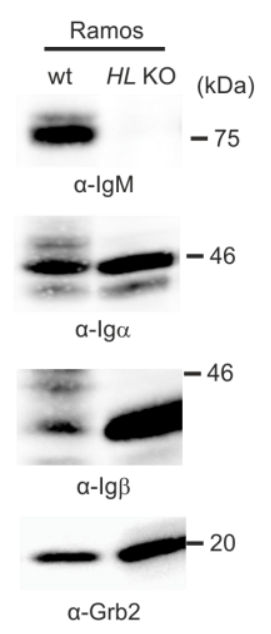

C

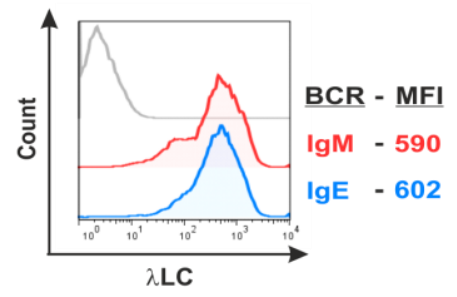

B

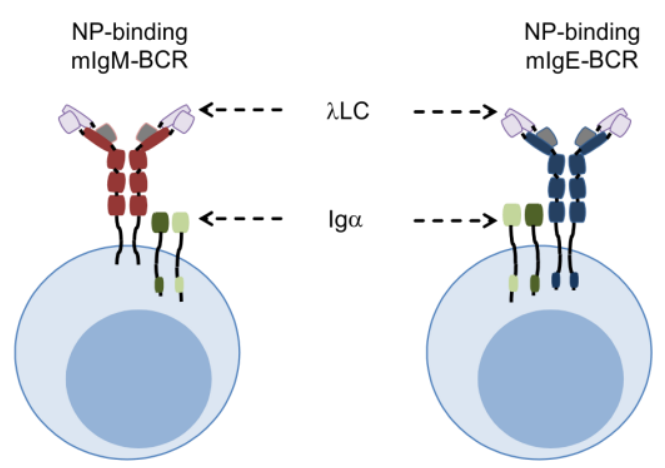

D

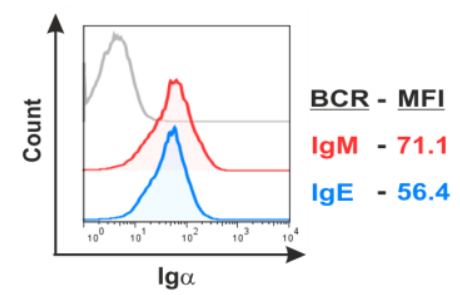

E

Normalized ratio of $\lambda L C: \lg \alpha$

IgM - 1.00

$\lg \mathrm{E}-1.27$

Figure 21: The human mlgE molecule can pair with $\lg \alpha / \lg \beta$ heterodimer.

(A) Lysates from the human Burkitt lymphoma cell line, Ramos in wild-type (wt) and heavy chain/lambda light chain deficient $(H L K O)$ backgrounds were analyzed by SDS-PAGE and immunoblotting. The blot was probed with antibodies against human $\lg \mathrm{M}, \lg \alpha, \lg \beta$ and Grb2. (B) Experimental setup. The Ramos $H L$ KO cells were reconstituted with the mouse lambda-1 light chain by using a $\lambda 1$-IRES-GFP construct and sorting for GFPpositive cells. The cells were further reconstituted with either NP-specific human $\mu \mathrm{m}$ or $\varepsilon \mathrm{m}$ heavy chains giving rise to cells expressing either mlgM-BCRs or mlgE-BCRs with NP-reactivity. Flow cytometric analysis of surface lambda light chain $(\lambda L C)(C)$ and $\lg \alpha(D)$ for mlgM-BCR (lgM) or mlgE-BCR (IgE) expressing cells. The mean florescence intensity (MFI) is given on the right side of each panel. (E) The normalized ratio of $\lambda L C: \lg \alpha$ was calculated as indicated for mlgM- and IgE-BCRs. Data are representative of one experiment.

Next, I determined the surface levels of the lambda light chain (Fig. 21C) and Ig $\alpha$ (Fig. 21D) on cells expressing either mlgM or mlgE. It is known that mlgM associates with the $\lg \alpha / \lg \beta$ heterodimer in a $1: 1$ stoichiometry (Schamel and Reth 2000). With this information, one could calculate the normalized ratio of lambda light chain $(\lambda \mathrm{LC})$ versus $\lg \alpha$ and set it to 1.0 for the mlgM-BCR. The normalized ratio of $\lambda \mathrm{LC}$ versus $\lg \alpha$ for $\mathrm{mlg} E-$ BCRs was found to be 1.27 (Fig. 21E). This means that there were more $\lambda \mathrm{LC}$ molecules than $\lg \alpha$ molecules associated with the mlgE-BCR. In other words, about $21 \%$ of $\mathrm{mlg} E$ molecules that made it to the cell surface were not associated with $\lg \alpha$. These results show that mlgE-BCRs can associate with $\lg \alpha / \lg \beta$. However they also hint towards a population of $\mathrm{mlgE}$ molecules that may not be associated with $\lg \alpha / \lg \beta$ at the cell surface. 
A

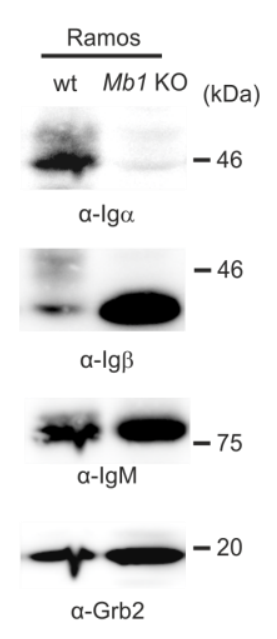

B

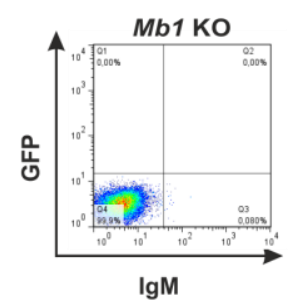

C

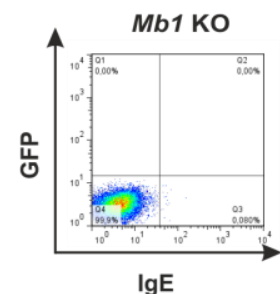

$\lg \mathrm{E}$

E

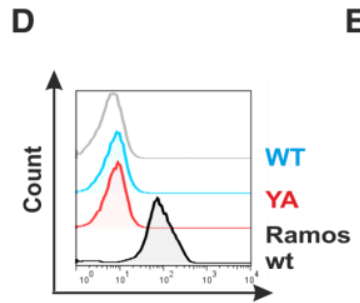

$\lg \beta$

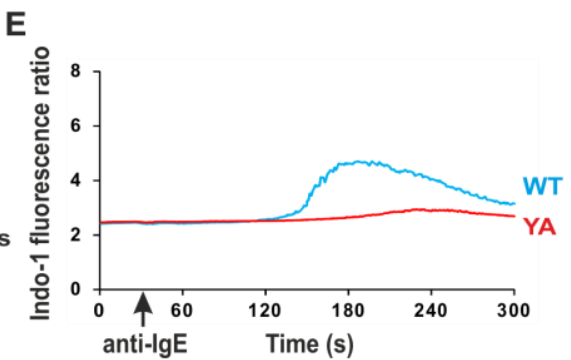

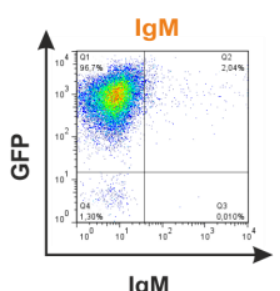

$\lg M$

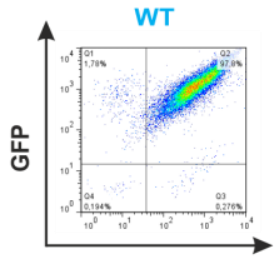

$\lg E$

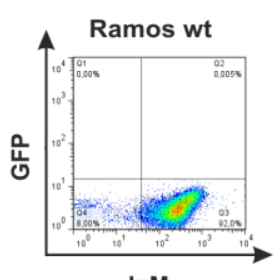

$\lg M$

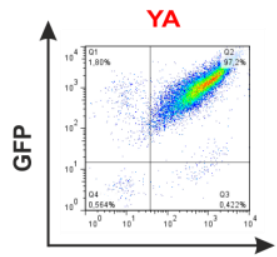

$\lg E$

F

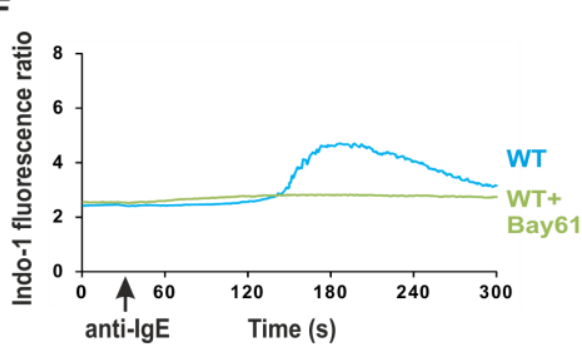

Figure 22: The human IgE molecule can be expressed on B cells as a functional signaling unit in the absence of the $\lg \alpha-\lg \beta$ heterodimer.

(A) Lysates from wild-type Ramos (wt) and Ig $\alpha$ deficient (Mb1 KO) Ramos cells were analyzed by SDS-PAGE and immunoblotting. The blot was probed with antibodies against $\lg \alpha, \lg \beta$, $\lg M$ and Grb2. (B and C) Ramos Mb1 KO cells were transfected with human- $\mu$ m-IRES-GFP, WT or ITT-YA mutant human- $\varepsilon$ m-IRES-GFP retroviral expression constructs and sorted for GFP-positive cells. (B) Surface mlgM vs. GFP levels were analyzed by flow cytometry (middle panel denoted as $\operatorname{lgM}$ ) along with untransfected and wild-type Ramos cells as controls (Left and right panels respectively). (C) Surface mlgE vs. GFP levels were analyzed by flow cytometry (middle and right panels denoted as WT and YA) along with untransfected cells (left panel). (D) Flow cytometric analysis of surface $\lg \beta$ on WT and ITT-YA mlgE-expressing Mb1 KO cells with wild-type Ramos cells (Ramos wt) as control. (E) $\mathrm{Ca}^{2+}$ mobilization in Ramos Mb1 KO cells expressing the WT and ITTYA mlgE molecules upon stimulation with $10 \mu \mathrm{g} / \mathrm{ml}$ anti-human-lgE antibody. (F) $\mathrm{Ca}^{2+}$ mobilization in Ramos Mb1 KO cells expressing the WT mlgE molecule in the absence (WT) or presence of $30 \mu \mathrm{M}$ Bay61-3606 Syk kinase inhibitor (WT+Bay61) following stimulation with $10 \mu \mathrm{g} / \mathrm{ml}$ anti-human-lgE antibody. Data are representative of one $(A, B, C$ and $D)$ or three $(E$ and $F)$ independent experiments.

To test whether a functional mlgE molecule can be expressed on the cell surface without the $\lg \alpha / \lg \beta$ heterodimer, I used Ramos cells deficient in Ig $\alpha$ (Ramos Mb1 KO) (Kindly provided by the lab of Michael Reth, Freiburg). These cells lack Ig $\alpha$ expression but express intracellular $\lg \beta$ and the $\mu$ heavy chain (Fig. 22A). However, due to the absence of $\lg \alpha$, a functional mlgM-BCR cannot be assembled and hence these cells lack surface mlgM (data not shown). I introduced the same NP-specific $\mu \mathrm{m}$ and $\varepsilon \mathrm{ms}$ heavy chains described above into the Ig $\alpha$-deficient Ramos cells. The heavy chains were introduced as IRES-GFP constructs to facilitate sorting for GFP-positive transfectants. There was no 
mlgM detected on the surface in the absence of $\lg \alpha$ (Fig. 22B). However, the human mlgE (WT and ITT-YA mutant) were efficiently expressed on the surface of these cells (Fig. 22C). The mlgE-expressing cells were also confirmed to lack surface $\lg \beta$ expression eliminating the possibility of $\mathrm{m} \lg E-\lg \beta-\lg \beta$ complexes (Fig. 22D). These data show for the first time that a transmembrane segment containing mlg molecule, namely human mlgE, can be expressed on the surface of $B$ cells without the $\lg \alpha / \lg \beta$ heterodimer.

I also analyzed the functional capabilities of the mlgE-ITT in the lg $\alpha$-deficient Ramos cells and found that the ITT could still mobilize $\mathrm{Ca}^{2+}$ in the absence of $\lg \alpha$ and $\lg \beta$, albeit to a lesser extent than in wild-type DG75 cells where $\lg \alpha-\lg \beta$ are present (Fig. 22E, compare to Fig. 19A). Subsequently, I analyzed whether the $\lg \alpha-\lg \beta$-independent activation of the ITT required the catalytic activity of Syk because traditionally Syk is known to be recruited to the BCR via the ITAMs. Interestingly, inhibition of Syk function using a Syk inhibitor completely abolished mlgE-ITT signaling (Fig. 22F). Thus, human mlgE can be expressed on the surface of $B$ cells in the absence of $\lg \alpha / \lg \beta$ and mediates some signaling which is dependent on the kinase Syk. Additionally, this brings to light a novel recruitment mechanism of Syk to the $\mathrm{mlg} E$ in the absence of the $\lg \alpha / \lg \beta$ ITAMs.

To analyze which region of the mlgE molecule imparted it with the ability to be expressed in the absence of $\lg \alpha / \lg \beta$, I equipped mlgM with certain segments of mlgE. One chimera was generated by replacing only the cytoplasmic tail of $\mathrm{mlgM}$ with that of $\mathrm{mlgE}(\mathrm{mlgM} / \mathrm{E}$ Cyt). Another chimera consisted of mlgM containing the $\mathrm{mlgE}$ transmembrane region and cytoplasmic tail (mlgM/E-TM+Cyt). Amino acid sequences and schematic representations of the chimeras along with the wild-type $\mathrm{mlgM}$ and $\mathrm{mlgE}$ molecules are depicted in Fig. $23 \mathrm{~A}$ and $\mathrm{B}$ respectively. I expressed these chimeric mlgs in the Ramos Ig $\alpha$-deficient cells to test whether the transmembrane or cytoplasmic region can provide a gain of function property to the mlgM molecule allowing $\lg \alpha / \lg \beta$-independent expression. I found that both chimeras, $\mathrm{mlgM} / \mathrm{E}-\mathrm{Cyt}$ and $\mathrm{mlgM} / \mathrm{E}-\mathrm{TM}+\mathrm{Cyt}$, could be expressed on the cell surface without $\lg \alpha$ and $\lg \beta$ (Fig. 23C). Lack of wild-type mlgM expression provided a good negative control in this setting. Note that the BCR-negative populations in the expression profiles of $\mathrm{mlgM} / \mathrm{E}-\mathrm{Cyt}, \mathrm{mlgM} / \mathrm{E}-\mathrm{TM}+\mathrm{Cyt}$ and $\mathrm{mlgE}$ represent untransfected cells that did not die after treatment with the selection antibiotic. Equipping the mlgM molecule with the cytoplasmic tail segment from human mlgE is sufficient to express mlgM on the cell surface in an $\lg \alpha / \lg \beta$-independent manner. Moreover, the mlgE transmembrane segment does not seem to be essential for this process. Thus, the cytoplasmic tail of the human $\mathrm{mlg} E$ molecule provides this isotype with the capability to be expressed without $\lg \alpha / \lg \beta$. 
A

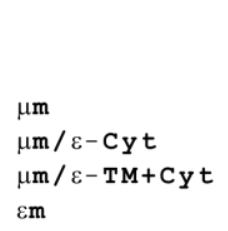

B

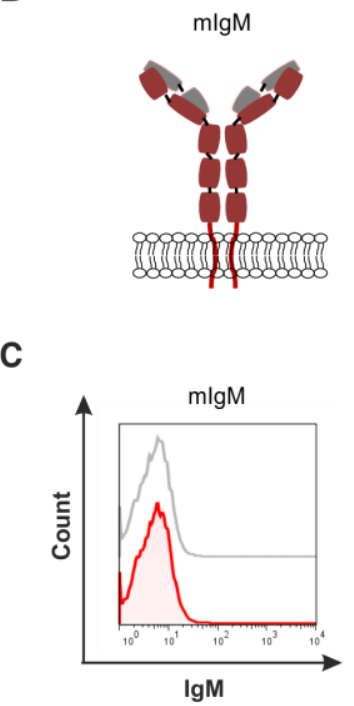

C

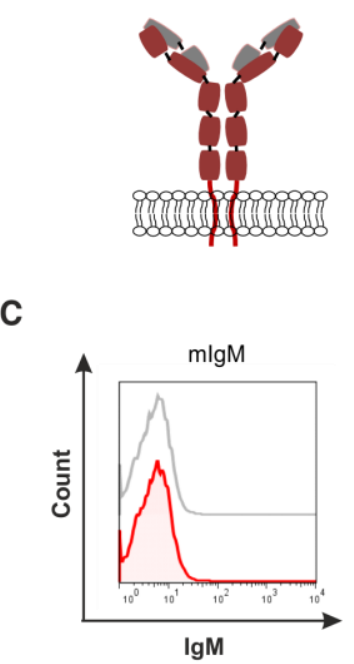

Transmembrane region

WATASTFIVLFLLSLFYSTTVTLFKVK

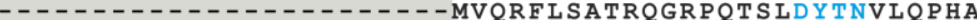
WTGLCIFAALFLLSVSYSAALTLLMVQRFLSATRQGRPQTSLDYTNVLQPHA

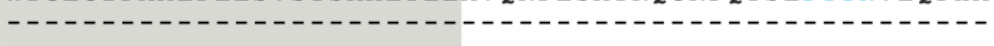

Cytoplasmic region
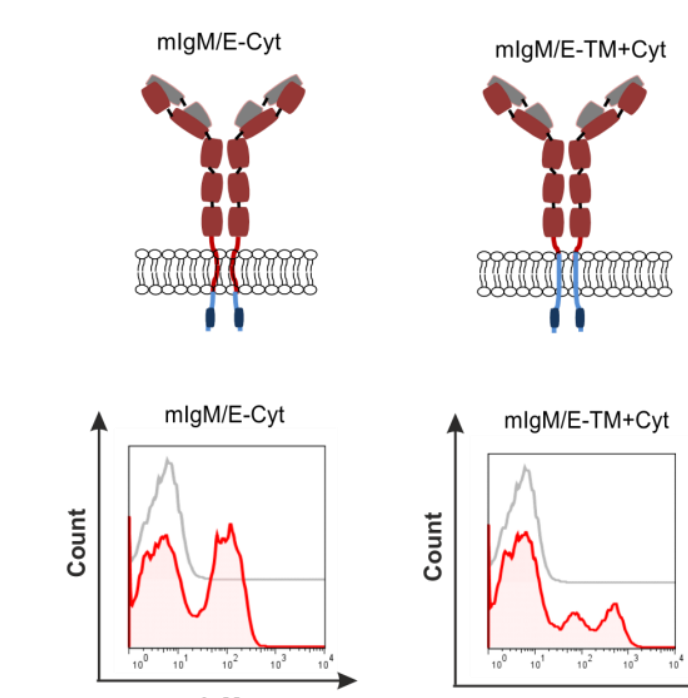

$\lg M$
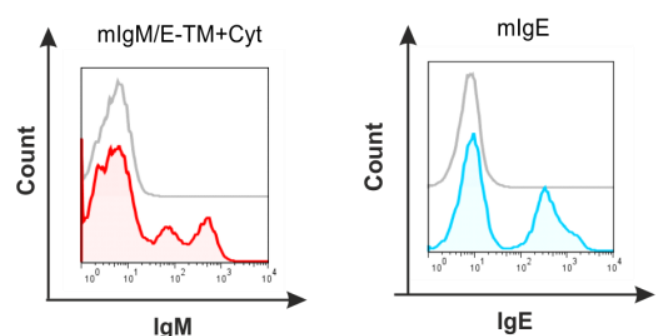

Figure 23: The cytoplasmic tail of human $\mathrm{mlgE}$ is sufficient to mediate $\lg \alpha / \lg \beta$-independent expression.

(A) Single letter amino acid sequence alignment of the transmembrane (TM) and cytoplasmic (Cyt) regions of the human $\mathrm{mlgM}(\mu \mathrm{m})$ and $\mathrm{mlgE}(\varepsilon \mathrm{m})$ variants used. The transmembrane region is marked in grey and the mlgE-ITT motif is highlighted in blue (B) Experimental setup. The cytoplasmic tail alone or the transmembrane and cytoplasmic regions of human mlgE were fused to the human mlgM heavy chain to produce chimeras called $\mathrm{mlgM} / \mathrm{E}-\mathrm{Cyt}$ or $\mathrm{mlgM} / \mathrm{E}-\mathrm{TM}+\mathrm{Cyt}$ respectively. Using the wild-type mlgM and mlgE molecules as controls, the chimers were retrovirally transduced into Ramos Mb1 KO cells. (C) Surface expression of mlgM, $\mathrm{mlgM} / \mathrm{E}-\mathrm{Cyt}, \mathrm{mlgM} / \mathrm{E}-\mathrm{TM}+\mathrm{Cyt}$ and $\mathrm{mlgE}$ was analyzed by flow cytometry. Data are representative of two independent experiments.

Finally, there have been some reports of mlgE expression in mouse and human plasma cells in vivo (Yang et al 2012, He et al 2013, Talay et al 2014, Ramadani et al 2016). It was not tested whether mlgE is expressed with or without $\lg \alpha$ or $\lg \beta$. Normally, plasma cells lose expression of the $\mathrm{mlg}$ (at least $\lg \mathrm{M}, \lg \mathrm{G}$ and $\lg A$ secreting plasma cells) and instead produce large amounts of the secreted antibody. Thus, I decided to analyze whether human mlgE can be expressed on the surface of plasma cells in the absence of $\lg \alpha / \lg \beta$. I used the mouse plasma cell line J558L to introduce the human $\mu \mathrm{m}$ and human ems heavy chains. J558L cells have lost the capability to produce an endogenous heavy chain and $\lg \alpha$ and express some intracellular $\lg \beta$ and the lambda light chain (Hombach et al 1990). Upon analysis of transfected cells, I found that mlgM could not be expressed on the surface of these cells (Fig. 24A). On the other hand, human mlgE is expressed on the cell surface of J558L plasma cells (Fig. 24B). To confirm intracellular $\mu \mathrm{m}$ expression in these cells, I analyzed cell lysates by immunoblotting for human IgM (Fig. 24C). Thus, human $\mathrm{mlgE}$, but not $\mathrm{mlgM}$, can be expressed on the surface of plasma cells in the absence of the $\lg \alpha / \lg \beta$ heterodimer. 
Results

A

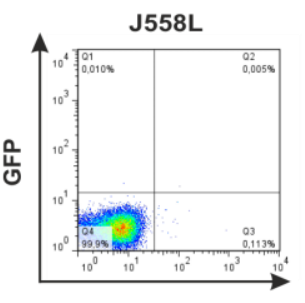

$\lg M$

B

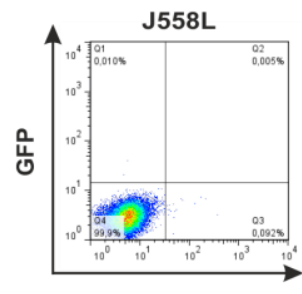

$\lg$

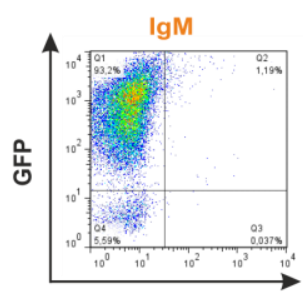

$\lg M$

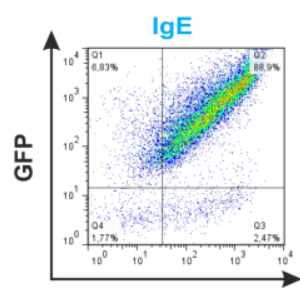

$\lg \mathrm{E}$
C

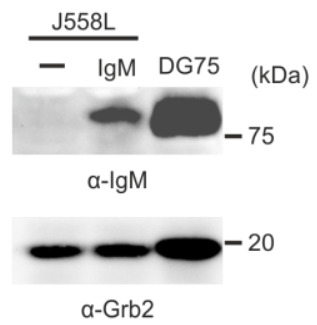

Figure 24: The human mlgE molecule can be expressed on terminally differentiated plasma cells.

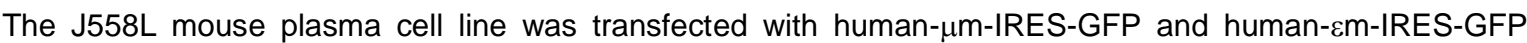
retroviral expression constructs and sorted for GFP-positive cells. GFP versus surface mlgM (A) and mlgE (B) expression was analyzed by flow cytometry. Untransfected J558L cells were used as negative control for mlgM and mlgE staining. (C) Intracellular mlgM expression in J558 transfected with mlgM was tested by analyzing cell lysates by SDS-PAGE and immunoblotting with an anti-IgM antibody. Parental J558L cells (-) and human DG75 B cells were used as controls. Grb2 served as a loading control. Data are representative of one experiment.

In conclusion, the human mlgE molecule is very distinct from mlgM in that it can be expressed on $B$ cells in the absence of the $\lg \alpha / \lg \beta$ heterodimer, a feature that is imparted by the cytoplasmic tail of mlgE. Moreover, the mlgE-ITT can signal in the absence of $\lg \alpha / \lg \beta$ via a novel Syk-dependent mechanism, the physiological role of which would be of great interest for future investigations. 


\section{Discussion}

The biology of $\lg E$ memory $B$ cell responses is only beginning to be understood. The scarce nature of "true" mlgE-expressing memory B cells makes them extremely difficult to analyze in vivo. However, the phase in the life of an $\lg E$ memory $B$ cell where it expresses a mlgE-BCR is important for the generation of $\lg E$ antibody responses as is evidenced by the lack of serum $\lg E$ in mice that cannot express mlgE (Achatz et al 1997). Therefore, examining mlgE-BCR signaling is a critical starting step towards uncovering the mechanisms that regulate $\operatorname{lgE}$ memory. To understand the composition and signaling properties of mlgE-BCRs in detail, I studied human mlgE in B cell lines. Using an anti-lgE antibody as a substitute for antigen, I could study the intracellular signaling mechanisms of mlgE-BCRs following their stimulation. I made the following key observations in the course of my work:

- The ITT motif in the cytoplasmic tail of the mlgE molecule is the principle signal amplification device of mlgE-BCRs.

- The mlgE-ITT reduces the threshold for activation of mlgE-BCRs, leading to enhanced $\mathrm{Ca}^{2+}$ mobilization and ERK MAP kinase activation.

- Upon phosphorylation by the tyrosine kinase Syk, the adaptor proteins Grb2 and Grap are recruited to the mlgE-ITT and can independently contribute to ITT-mediated signal amplification.

- Grb2 and Grap are also required for optimal canonical ITAM-induced $\mathrm{Ca}^{2+}$ signaling of mlgM-BCRs via their effect on activation of the $\mathrm{Ca}^{2+}$ initiation complex proteins.

- Unlike mlgM, the human mlgE molecule can be expressed on the B cell surface in the absence of $\lg \alpha$ and $\lg \beta$, a feature which is dependent on the mlgE cytoplasmic tail.

- The mlgE-ITT can signal independently of the $\lg \alpha / \lg \beta$ heterodimer in a Syk-dependent manner.

\subsection{BCR signal amplification by the ITT signaling module in the context of mlgE-BCRs.}

The ITT motif was previously described as a BCR signal amplification device present in the cytoplasmic tail of $\mathrm{mlgG}$, which also augments serum IgG1 production in vivo (Engels et al 2009, Lutz et al 2015). The mammalian mlgG and mlgE $\lg$ isotypes are separated from their common ancestor, mlgY by a time span of approximately 200 million years (Flajnik 2002). Despite the evolutionary distance, the ITT motif core comprising the YxN amino acid sequence is completely conserved in these mlg isotypes. I analyzed the ITT motifs in the mlg cytoplasmic tails of these isotypes by comparing the wild-type ITTcontaining BCR to ITT-YA mutant variants. My work revealed that all these isotypes contain a functional ITT motif which can mediate BCR signal amplification as measured by enhanced $\mathrm{Ca}^{2+}$ mobilization. The evolutionary conservation of ITT-mediated signaling in 
mlgG, mlgE and even mlgY memory-type BCRs emphasizes the importance of this process in B cells (Engels et al 2014). Furthermore, in the context of mlgE-BCRs, I demonstrated that the ITT is the only BCR signal amplification device in the cytoplasmic tail of mlgE, since deletion of most of the tail led to the same impairment in BCR signaling as the ITT-YA mutation. Combined, these observations prove that the ITT motif is the predominant signal amplification device in the cytoplasmic tail of memory-type BCRs.

Monitoring $\mathrm{Ca}^{2+}$ mobilization is a commonly used readout for $\mathrm{B}$ cell activation since this pathway leads to the activation of proteins that are involved in B cell activation, proliferation and differentiation (Feske 2007, Baba and Kurosaki 2011). Additionally, the ERK MAP kinase pathway is also critical for $B$ cell activation and differentiation into plasma cells (Yasuda et al 2008, Yasuda et al 2011). I observed that the mlgE-ITT improves activation of both of these signaling pathways. Furthermore, the ITT motif increases the sensitivity of mlgE-BCRs towards antigen, leading to prompt activation of $\mathrm{BCR}$ signaling pathways even at low antigen concentrations. In an in vivo situation, this would provide mlgE-expressing $B$ cells with a competitive advantage over cells lacking an ITT motif as the mlgE-ITT would allow swifter B cell activation. mlgE-expressing GC B cells have been described to rapidly undergo differentiation into plasma cells (Yang et al 2012). Although the in vivo significance of the mlgE-ITT is yet to be examined, one could envision that the ITT would help in the preferential activation and differentiation of mlgEexpressing $B$ cells thereby augmenting IgE responses.

I could also show that mlgE-ITT phosphorylation requires the catalytic activity of the tyrosine kinase Syk. Phosphorylation by Syk leads to the generation of a phosphotyrosine binding site for SH2 domain-containing proteins like Grb2, making it the most likely candidate to connect the mlgE-ITT with downstream signaling pathways. Unexpectedly, the mlgE-ITT did not completely lose its signal amplification capabilities in GRB2-deficient B cells, which indicated the presence of a second direct interaction partner for the ITT. Analysis of the ITT motif core sequence $(\mathrm{YxN})$ in more detail suggested that the tyrosine and asparagine residues are critical for mlg-ITT function. This led me to discovering Grap, a Grb2 family member that also prefers a phospho-YxNcontaining binding sequence, as a second functional interaction partner for the mlgE-ITT. The wild-type mlgE-BCR could not amplify BCR signaling in GRB2/GRAP double-deficient cells and reconstitution with either or both adaptor proteins could rescue the phenotype by reviving mIgE-ITT signal amplification. In summary, I found that both Grb2 and Grap are utilized by mlgE-BCRs for signal amplification via the ITT.

Our group previously demonstrated that the mlgG-ITT signals exclusively via the adaptor protein Grb2. Grb2 equips the mlgG-ITT with signal amplification properties by recruiting the kinase Btk to the ITT signalosome. The N-terminal SH3 domain of Grb2 can bind Btk whereas Grap cannot (Engels et al 2014). Therefore, although the mlgG-ITT can bind to Grap, the inability of Grap to recruit Btk makes it dispensable for mlgG-ITT signaling. 
So then how does Grap enable ITT-mediated signal amplification in the context of mlgEBCRs? There are some Btk-independent mechanisms operating in the context of both mlgG- and mlgE-BCRs as is evidenced by some residual ITT signaling in BTK-deficient B cells (Engels et al 2014, data not shown). It is possible that these mechanisms are more prevalent in the context of mlgE-BCRs making Grap as effective as Grb2 in mlgE-ITTmediated signal amplification. In agreement with this hypothesis is my observation that the Btk-binding N-terminal SH3 domain of Grb2, when mutated, can still enhance mlgE-BCR induced $\mathrm{Ca}^{2+}$ signaling as efficiently as wild-type Grb2. It is also important to note that the cytoplasmic tail of mlgE and mlgG are highly conserved at the ITT motif but the rest of the tail segment shows quite some variation. These unique amino acid residues of the $\mathrm{mlg} E$ tail could contribute to recruitment of certain proteins that support Grb2 and Grap in carrying out their function at the mlgE-ITT. Even though the exact mechanisms need future investigation, to my knowledge this is the first study to implicate Grap as a positive regulator of $\mathrm{BCR}$-mediated $\mathrm{Ca}^{2+}$ signaling.

Furthermore, I observed that cytosolic $\mathrm{Ca}^{2+}$ mobilization was not the only process that was affected by the absence of Grb2 and Grap, but mlgE-BCR-induced ERK activation was also severely impaired. The contribution of Grb2 and Grap in the activation of ERK is easier to understand since both proteins can bind to the GEF, Sos (Li et al 1993, data not shown), which can then initiate ERK activation via the Ras-MAPK pathway (Buday et al 1994, Kumar et al 1995). Therefore, I could show that Grb2 and Grap couple the mlgEITT to the ERK MAP kinase signaling pathway.

In conclusion, the mlgE-ITT upon phosphorylation by Syk, recruits the adaptor proteins Grb2 and Grap to mlgE-BCRs, leading to enhanced $\mathrm{Ca}^{2+}$ signaling and improved activation of the ERK MAPK pathway (see Fig. 25). This equips mlgE-expressing B cells with the chance to be activated even at low concentrations of antigen. Although $\lg E$ memory B cells are scarce, prompt activation of these cells via their high sensitivity BCRs would nevertheless amount to a biologically functional level of $\lg E$ responses against pathogens like helminths.

\subsection{The complex role of adaptor proteins in BCR signaling - regulation of ITAM signaling by Grb2 and Grap.}

A mlgM-BCR consists of a mlgM molecule associated non-covalently with the $\lg \alpha / \lg \beta$ heterodimer. The mlgM contains a very short cytoplasmic tail without any known signaling capabilities (Reth 1992). Therefore, antigen-induced mlgM-BCR signaling is solely mediated by the ITAMs in the cytoplasmic tails of $\lg \alpha$ and $\lg \beta$ (Kim et al 1993). Analysis of mlgM-BCR signaling in the GRB2/GRAP double-deficient cells revealed that the absence of these adaptors impairs optimal activation of canonical ITAM-induced $\mathrm{Ca}^{2+}$ mobilization. 


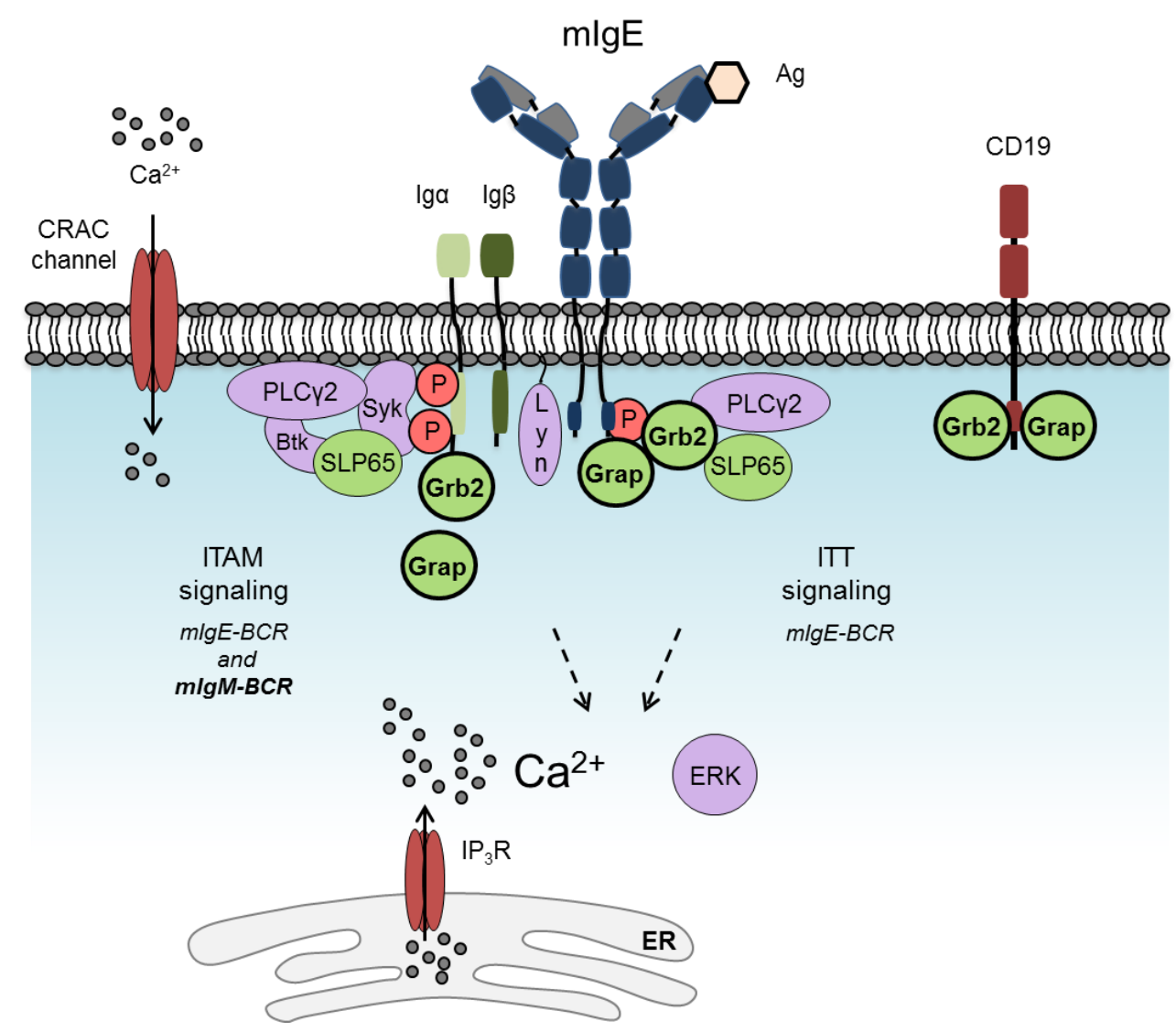

Figure 25: Grb2 and Grap are involved in mlgE-ITT-mediated BCR signal amplification and also contribute to canonical ITAM-mediated $\mathrm{Ca}^{2+}$ signaling of $\mathrm{mlgE}$ - and $\mathrm{mlgM-BCRs}$.

Illustration depicting the findings of this study regarding the molecular mechanisms dictating mlgE-ITT function and the role of Grb2 and Grap in canonical ITAM signaling. Upon activation of the mlgE-BCR, the mlgE-ITT is phosphorylated by the tyrosine kinase Syk. This leads to recruitment of the adaptor protein Grb2 and Grap, both of which can mediate mlgE-BCR signal amplification via enhanced $\mathrm{Ca}^{2+}$ mobilization and ERK MAP kinase activation. Additionally, the optimal activation of the $\mathrm{Ca}^{2+}$ initiation complex proteins Syk, SLP65 and PLC $\gamma 2$ requires the presence of Grb2 and Grap, thereby promoting cytosolic $\mathrm{Ca}^{2+}$ flux via ITAMs. Grb2 may be recruited to $\lg \alpha$, whereas the localization of Grap at the BCR remains unknown. Both proteins may additionally be recruited to the membrane via binding to the co-stimulatory molecule CD19 following BCR stimulation. Dashed lines represent indirect effects and solid line represent direct effects. Purple-kinases, green-adaptor proteins and $\lg \alpha / \lg \beta$ heterodimer, red-CD19 and $\mathrm{Ca}^{2+}$ channels, blue-mlgE, beige-antigen $(\mathrm{Ag})$, grey dots- $\mathrm{Ca}^{2+}$, -phosphorylation.

This is accompanied by reduced phosphorylation and thus activation of the BCR-proximal signal effector proteins Syk, SLP65 and PLC $\gamma 2$ that control the $\mathrm{Ca}^{2+}$ signaling pathway. Analysis of the individual domains of Grb2 and Grap brought me a step closer to understanding their functional contribution to the $\mathrm{Ca}^{2+}$ signaling pathway. The $\mathrm{SH} 2$ domain is required for $\mathrm{Ca}^{2+}$ signaling since it most likely recruits these adaptors to the active BCR signalosome. In regard to this, I could identify the BCR itself (Grb2) or to the co-stimulatory molecule CD19 (Grb2 and Grap) as binding sites for the adaptors following BCR stimulation. Upon mlgM-BCR stimulation, the N-terminal SH3 domains of Grb2 and 
Grap are critical for $\mathrm{Ca}^{2+}$ mobilization. On the other hand, mlgE-BCR induced $\mathrm{Ca}^{2+}$ flux utilizes either Grb2 SH3 domain but is reliant on a functional N-terminal SH3 domain of Grap. The functional differences displayed by the $\mathrm{SH} 3$ domains at the mlgM- and mlgEBCRs imply that there are different routes used to activate $\mathrm{Ca}^{2+}$ signaling by different $\mathrm{BCR}$ isotypes. This may be partly explained by the fact that compared to mlgM-BCRs, which only have the ITAMs in $\lg \alpha / \lg \beta$, mlgE-BCRs also contain an ITT motif and hence Grb2 and Grap could be employing different molecular mechanisms at these two motifs to activate the $\mathrm{Ca}^{2+}$ signaling pathway. In conclusion, the functional role of Grb2 and Grap is not limited to the mlgE-ITT (as discussed in the previous section), but also extends to the canonical ITAM-signaling pathway.

I can propose some explanations as to how Grb2 and Grap might positively modulate ITAM-induced $\mathrm{Ca}^{2+}$ signaling. One explanation is that Grb2 and Grap act upstream of Syk in the BCR signaling cascade and thereby affect activation of the $\mathrm{Ca}^{2+}$ initiation complex. In fact, it has been reported that in T cells, Grb2 acts at a very early stage of T cell antigen receptor signaling by positively modulating the activity of the tyrosine kinase Lck (Jang et al 2010), which is the T cell functional homologue of Lyn in B cells. A similar concept might be applicable to B cells, where Grb2 and Grap help in optimal Lyn-mediated activation of Syk, PLC $\gamma 2$ and SLP65. The main biological role of adaptor proteins is to nucleate signalosomes by recruiting and/or stabilizing protein complexes. Both Grb2 and Grap can at least associate directly with SLP65 (Engels et al 2014), though the complete interactome of Grb2 and Grap in human B cells needs elucidation. The presence of Grb2 and Grap at the signalosome could additionally increase the local concentration of signaling proteins recruited by these adaptors by virtue of their $\mathrm{SH} 3$ domains. This would then help in the optimal activation of the recruited proteins and simultaneously facilitate signal amplification as well.

The recruitment of Grb2 and Grap to the co-stimulatory molecule CD19 (this study) may also be functionally relevant. Co-ligation of CD19 with the mlgM-BCR has been shown to increase BCR-induced $\mathrm{Ca}^{2+}$ signaling (Fujimoto et al 2001). CD19 induces activation of $\mathrm{PI} 3 \mathrm{~K}$, a protein that regulates $\mathrm{Ca}^{2+}$ signaling by increasing the local concentration of $\mathrm{PIP}_{3}$ at the plasma membrane, which then serves as membrane anchor for many BCR signaling proteins including Btk and PLC $\gamma 2$ (Buhl and Cambier 1999, Jou et al 2002, Okkenhaug and Vanhaesebroeck 2003). Additionally, interaction of the Btk PH domain with $\mathrm{PIP}_{3}$ has also been associated with allosteric activation of Btk kinase activity (Saito et al 2001). How PI3K is activated following BCR stimulation is not completely understood. It would be interesting to analyze whether Grb2 and Grap can modulate PI3K activity downstream of the BCR and CD19 and could hence indirectly affect Btk and PLC $\gamma 2$ activity. 
The functional role of Grb2 has not been well studied in B cells, accompanied by almost no insight into the function of Grap. The current standing in the field is that Grb2 negatively regulates mlgM-BCR signaling. This is based on some reports that observed enhanced $\mathrm{Ca}^{2+}$ flux in the absence of Grb2 in either a chicken B cell line or mouse models (Stork et al 2004, Ackermann et al 2011, Jang et al 2011). It is important to point out that Grb2 also interacts with proteins like SH2 domain-containing inositol phosphatase 1 (SHIP1), CD22 and downstream of tyrosine kinase 3 (Dok3), all of which are negative regulators of BCR signaling (Neumannn et al 2009, Stork et al 2007, Poe et al 2000). However, my data presents a positive role for Grb2 (and Grap) in BCR-induced $\mathrm{Ca}^{2+}$ signaling in human B cells. Although I cannot exclude species-specific differences influencing the function of Grb2, the high degree of homology between the human and mouse orthologs makes functional conservation seem quite likely. B cell-specific Grb2deficient mice show considerable impairment in the development of mature $B$ cells (Ackermann et al 2011, Jang et al 2011). Given that proper BCR signaling is critical for normal B cell development (Melchers et al 1993, Reth and Nielson 2014), these mice perhaps never received signals required to give rise to a normal $B$ cell compartment leading to hyper-responsiveness on BCR stimulation. B cell maturation phase-specific conditional Grb2-deficient mice would perhaps provide clearer answers to whether Grb2 also plays a positive role in mlgM-BCR signaling in mice.

In contrast to the supposed negative role of Grb2 in BCR signaling, Grb2-deficient mice display reduced serum IgG titers (Ackermann et al 2011). Supporting this is the observation that activation of lgG-switched memory B cells and their differentiation into IgG-secreting cells requires Grb2 in vivo (Engels et al 2014). Thus, it is possible that Grb2 has a more complex role in regulating BCR signaling, where it can play a positive or a negative role depending on the site of recruitment - the BCR, CD19, CD22 or Dok3.

Unlike $\mathrm{Ca}^{2+}$ signaling, I found that BCR-induced activation of ERK requires all three domains of Grb2 and Grap to be functional. This can be explained by the fact that binding of Sos to both Grb2 SH3 domains stabilizes this interaction as compared to the low affinity binding to only one of the SH3 domains (Simon and Schreiber 1995). Moreover, it is also important to highlight the fact that the absence of Grb2 and Grap leads to an almost complete block in ERK activation whereas $\mathrm{Ca}^{2+}$ signaling is not as drastically reduced (see Fig. 16 and 17). These observations imply that $\mathrm{Ca}^{2+}$ and ERK signaling pathways are not directly coupled as their activation require different domains of the adaptors to be functional and that perhaps different pools of Grb2 and Grap diversify the initial BCR signal either into the $\mathrm{Ca}^{2+}$ pathway or the ERK pathway.

Based on the data from this study, I propose that Grb2 and Grap can positively regulate ITAM-induced $\mathrm{Ca}^{2+}$ signaling in human B cells (see Fig. 25). The utilization of different adaptor proteins like Grb2 and Grap by the BCR probably helps improve B cell activation via both signal amplification as well as signal diversification. 


\subsection{It all comes down to the tail - human mIgE expression and ITT signaling in the absence of $\lg \alpha / \lg \beta$.}

A single unit of the mlgM-BCR consists of mlgM and the $\lg \alpha / \lg \beta$ heterodimer in a $1: 1$ stoichiometry wherein the signaling function is mediated by the ITAMs in the cytoplasmic tails of $\lg \alpha$ and $\lg \beta$ (Schamel and Reth 2000, Kim et al 1993). Similarly, the mlgE-BCR is believed to be composed of $\mathrm{mlg} E$ plus $\lg \alpha / \lg \beta$, with the exception that the longer $\mathrm{mlg} E$ cytoplasmic tail has an additional ITT motif signaling module. The mlgE-ITT is completely non-functional in the absence of the adaptor molecules Grb2 and Grap (this study). Therefore, in the absence of Grb2 and Grap, the mlgE-BCR should only signal via the ITAMs in $\lg \alpha$ and $\lg \beta$, making its signaling capabilities similar to that of mlgM-BCRs. Surprisingly, this was not the case, since I could observe a much stronger impairment in $\mathrm{Ca}^{2+}$ signaling for mlgE-BCRs compared to mlgM-BCRs in GRB2/GRAP double-deficient cells. This led me to investigate the ITAM signaling pathway in mlgE-BCRs and I found that in DG75 B cells, there was very little activation of ITAMs following mlgE-BCR stimulation as exemplified by the lack of $\lg \alpha$ phosphorylation. Moreover, I could barely copurify any $\lg \alpha$ and $\lg \beta$ with surface $\mathrm{mlg} E$ whereas surface $\mathrm{mlgM}$ associated with substantial amounts of these proteins under identical buffer conditions.

One possible explanation for the above mentioned observations would be active competition between mlgM and mlgE for the $\lg \alpha / \lg \beta$ heterodimer. DG75 B cells normally express only mlgM and perhaps introduction of an additional mlgE molecule creates an overload for the cellular machinery to produce sufficient $\lg \alpha / \lg \beta$ for both isotypes. However, additional expression of mlgE did not alter mlgM-BCR expression or signaling when compared to cells expressing only mlgM (data not shown). Nevertheless one could argue that the expression of two different isotypes may not be normal for a $\mathrm{B}$ cell in vivo. This is actually not true since, naïve $B$ cells can express both mlgM and mlgD on the same cell (Havran et al 1984). More specific to this situation, there are some reports of $\lg \mathrm{M}$ and $\lg \mathrm{E}$ co-expression in human $\mathrm{B}$ cells during on-going class-switch recombination although the mlg phenotype of these cells was not clearly analyzed (Mackenzie and Dosch 1989, Wesemann et al 2011). Therefore, mlgM and mlgE co-expression may not be as improbable as one would normally imagine.

An alternative explanation for the lack of ITAM signaling upon mlgE-BCR stimulation would be that there is no association between human $\mathrm{mlg} E$ and the $\lg \alpha / \lg \beta$ heterodimer. Since the DG75 B cells I used, expressed both BCR isotypes on the same cells, I could not analyze the $\lg \alpha / \lg \beta$ pools associated with the individual mlg isotypes. Therefore, I used an Ig heavy and Ig light chain deficient variant of Ramos B cells, which are human Burkitt lymphoma cells similar to DG75 B cells. Lack of endogenous heavy chain expression enabled independent expression of either mlgM or mlgE in these cells. I could show that both $\mathrm{mlg}$ isotypes were expressed with surface $\lg \alpha / \lg \beta$, although the ratio of 
mlg to $\lg \alpha$ was a bit more for mlgE-BCRs. Thus, although mlgE can associate with $\lg \alpha$ and $\lg \beta$, these results also hinted towards some $\lg \alpha / \lg \beta$-less $\mathrm{mlg} E$ molecules on the cell surface. Moreover, since the mlgE-ITT-YA and mlgE-tailless mutants are signaling competent in B cells, there is probably a pool of $\lg \alpha / \lg \beta$ associated with mlgE, mediating signaling via the ITAMs. Linking the observations, I conclude that mlgE can associate with $\lg \alpha / \lg \beta$ although there might also be a fraction of free surface mlgE in B cells.

To conclusively prove the existence of surface $\mathrm{mlg} E$ without $\lg \alpha / \lg \beta$, I used $\lg \alpha$-deficient Ramos B cells where the absence of $\lg \alpha$ also prevents surface expression of $\lg \beta$. Expression of human mlgE in these cells revealed that this mlg can be expressed as a stand-alone molecule without the $\lg \alpha / \lg \beta$ heterodimer and that the mlgE-ITT can signal in the absence of $\lg \alpha / \lg \beta$ in a Syk-dependent manner. In contrast, mlgM did not reach the cell surface and remained intracellular in the absence of Ig $\alpha$. Furthermore, I found that the cytoplasmic tail of $\mathrm{mlg} E$ is required for the $\lg \alpha / \lg \beta$-independent surface expression since fusing the mlgE cytoplasmic tail to mlgM was enough to express mlgM on the cell surface without $\lg \alpha / \lg \beta$.

Mechanistically, these results pose some important questions regarding the surface transport and signaling mechanisms utilized by the human mlgE molecule. For instance, does the mlgE cytoplasmic tail actively uncouple itself from the $\lg \alpha / \lg \beta$ heterodimer or is there an ER quality control escape mechanism that allows human mlgE to be transported to the cell surface without $\lg \alpha / \lg \beta$ ? mlgM-BCRs require all components including $\lg \alpha$ and $\lg \beta$, to be properly assembled in the ER for transport and expression on the cell surface through a not so well understood mechanism. Certain chaperones have been reported to associate with the Ig heavy and light chains and to affect their ER processing (Bole et al 1986, Knittler et al 1995). It would be interesting to investigate whether the short cytoplasmic tail sequence of IgM which contains only three amino acids KVK (single letter code), somehow acts as an ER retention signal in the absence of $\lg \alpha / \lg \beta$. The human mlgE cytoplasmic tail does not have this sequence, and instead contains the amino acid sequence MVQR (single letter code), followed by 24 additional residues which could lead to alternative processing mechanisms in the ER. In fact, when I expressed a variant of mlgE with only the MVQR residues in the cytoplasmic tail (tailless mlgE variant in Fig. 4), this molecule exhibited lower surface expression than wild-type mlgE. This suggests that some mlgE cytoplasmic tail-dependent regulatory mechanisms operate in B cells and require future investigation.

Another novel finding made by me was that the mlgE-ITT can mediate some $\mathrm{Ca}^{2+}$ signaling without $\lg \alpha / \lg \beta$ in a Syk-dependent manner. Traditionally, Syk is believed to be recruited to the BCR signaling complex via phosphorylated ITAMs (Johnsen et al 1995, Kurosaki et al 1995). The results from this study suggest a new mechanism of Syk recruitment to the $\mathrm{mlg}$, independently of ITAMs. Additionally, it is also relevant to 
investigate whether this signaling function of $\mathrm{mlgE}$ is completely self-dependent or whether some membrane/cytosolic interaction partner can compensate for the absence of $\lg \alpha / \lg \beta$ ? A very recent report implicated that the association between CD19 and mlgE promotes autonomous mlgE signaling which culminates in either apoptosis or plasma cell differentiation in mice (Haniuda et al 2016). Could CD19 be a surrogate for $\lg \alpha / \lg \beta$ in case of the human mlgE molecule? Preliminary work in this direction revealed that the human mlgE-BCR signals equally efficiently in wild-type and CD19-deficient B cells. Also, I could not detect any association between CD19 and human mlgE in biochemical experiments (data not shown). Another possibility could be that binding of Grb2 and/or Grap to the mlgE-ITT can initiate BCR signaling without $\lg \alpha / \lg \beta$ due to their ability to positively regulate $\mathrm{Ca}^{2+}$ signaling at a $\mathrm{BCR}$-proximal level, as revealed in this study. Future investigation into the mechanism by which the human mlgE-ITT can signal without $\lg \alpha / \lg \beta$ and whether CD19, Grb2 or Grap are involved in that process may provide some useful insights.

From a functional point of view, expression of $\mathrm{mlgM}$ in the absence if $\lg \alpha / \lg \beta$ would not make sense since the signaling function of this BCR isotype solely resides in the ITAMs in the cytoplasmic tails of the $\lg \alpha / \lg \beta$ heterodimer (Kim et al 1993). Hence, such a molecule would not be able to relay any intracellular signaling, even after antigen binding. The presence of the ITT in the cytoplasmic tail of mlgE endows some signaling capabilities to this isotype even in the absence of the $\lg \alpha / \lg \beta$ heterodimer in the GC-like Burkitt B cell lines used in this study. In a physiological setting, surface expression of mlgE in the absence of $\lg \alpha / \lg \beta$ would more likely occur in the last stage of B cell differentiation which is plasma cells. Plasma cells do not express any $\lg \alpha$ and also switch from expression of the mlg to the secreted Ig. I could show that human mlgE can be expressed on the surface of plasma cells. Even though the in vivo significance of this $\lg \alpha / \lg \beta$-independent ITT signaling process remains to be investigated, it is tempting to speculate that this kind of signaling module might affect the fate of mlgE-positive $B$ cells, modulating the formation/maintenance of $\lg E$ memory.

Remarkably, in-depth analysis of mlgE-positive B cells in mice showed that most of these cells were plasma cells (Yang et al 2012, Talay et al 2014). A recent study in which lgE plasma cells were generated using in vitro differentiation of human tonsillar B cells also reported mlgE expression on the surface of human plasma cells (Ramadani et al 2016). There is some evidence of long-lived IgE plasma cells in humans and that these cells may contribute to allergic responses (Hallstrand et al 2004, Mitre et al 2006). It would hence be crucial to analyze human mlgE-positive long-lived plasma cells in more detail to see if the mlgE molecule actively contributes to an ongoing $\lg E$ response via ITT signaling. Additionally, BCR signaling is not only required for antigen-induced activation but also survival of B cells in the absence of any antigen (Werner et al 2010). Therefore, it remains to be seen whether mlgE also provides survival signals to plasma cells. Answers to these 
questions would have useful implications in designing therapy to effectively target all mlgE-positive B cells for treating allergies.

Combining my observations, I suggest that the $\lg \alpha / \lg \beta$-independent mlgE-ITT signaling module hints toward a novel mechanism regulating the biological activity of the $\lg \mathrm{E}$ memory compartment.

\subsection{Conclusions and perspectives}

The biological role of $\operatorname{lgE}$ is to mediate clearance of large pathogens like helminths from the body. However, IgE also plays a clinically significant role since it is one of the main mediators of immediate hypersensitivity reactions. It is thus important to understand how IgE memory B cells are activated so this information can be applied to therapeutically target these cells in an effective manner. In this work, I described some molecular features of mlgE-BCR signaling, which contribute towards our understanding of $\lg \mathrm{E}$ memory responses. mlgE-BCRs contain the evolutionarily conserved ITT motif in the mlgE cytoplasmic tail, which increases the antigen sensitivity of these BCRs via utilization of the adaptor proteins, Grb2 and Grap, This leads to prompt activation of BCR-proximal signaling pathways in mlgE-expressing cells. Interestingly, the human mlgE cytoplasmic tail confers the molecule with the capacity to be expressed as a functional signaling unit in the absence of the $\lg \alpha / \lg \beta$ heterodimer (see Fig. 26 on next page).

To my knowledge, this is the first report of a mlg isotype having the capacity to relay intracellular signals even in the absence of the $\lg \alpha / \lg \beta$ heterodimer, which are considered necessary for BCR signal initiation based on current textbook models. It would be of extreme interest to determine whether this novel signaling mechanism influences the formation and/or maintenance of the memory lgE B cell compartment. Moreover, if it turns out that plasma cells also express this $\lg \alpha / \lg \beta$-independent signaling competent $\mathrm{mlg} \mathrm{E}$ molecule in vivo, it would be important to consider a more active contribution of mlgEpositive plasma cells in regulating IgE memory responses via mlgE-ITT signaling. 
Discussion

A

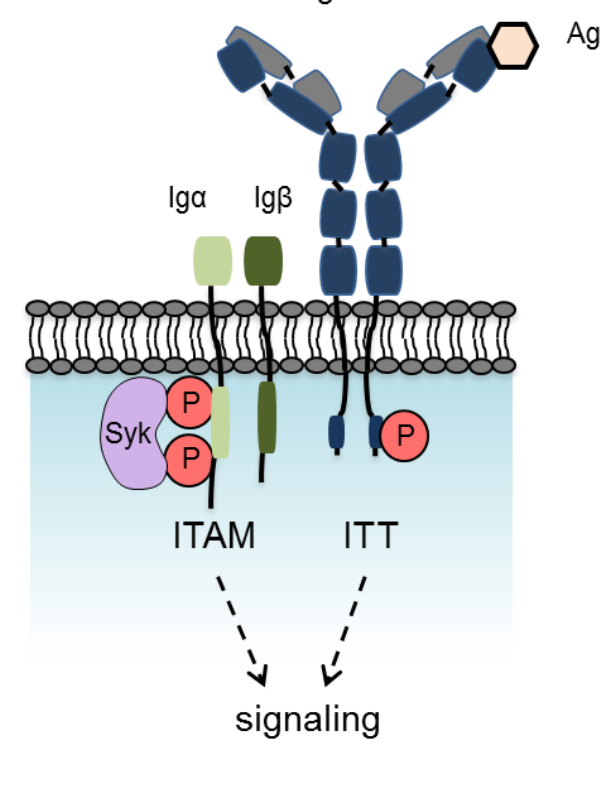

B

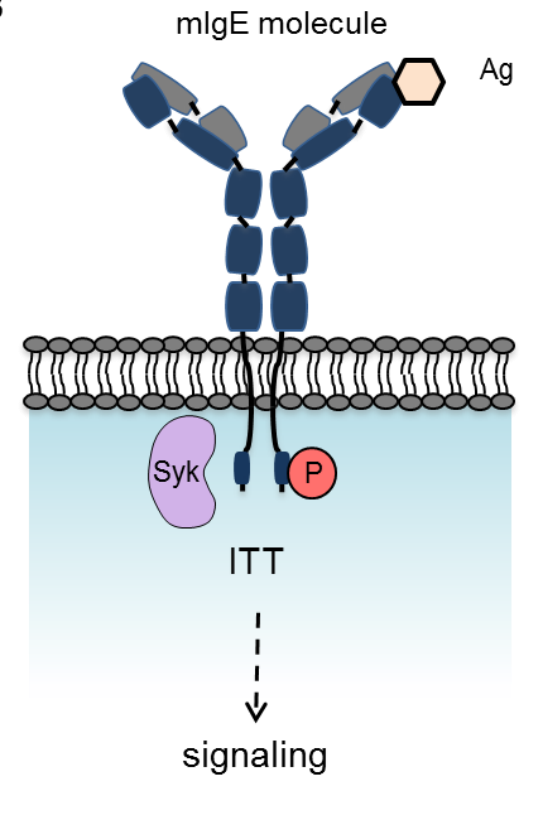

Figure 26: The human mlgE-ITT can function independently of the $\lg \alpha / \lg \beta$ heterodimer.

Illustration depicting the various signaling modules of human mlgE as elucidated in this study. (A) The conventional mlgE-BCR complex is composed of the ITAM signaling motifs in the $\lg \alpha / \lg \beta$ cytoplasmic tail and the ITT motif in the mlgE cytoplasmic tail. (B) The human mlgE can also be expressed on the B cell surface in the absence of $\lg \alpha / \lg \beta$. Activation of the mlgE molecule leads to mlgE-ITT mediated $\mathrm{Ca} 2+$ signaling which is dependent on the tyrosine kinase Syk. Such a module can be expressed on both GC-like B cells and plasma cells. Dashed lines represent indirect effects. Purple-tyrosine kinase Syk, green-Ig $\alpha / \lg \beta$ heterodimer, bluemlgE, beige-antigen (Ag), ๑-phosphorylation. 


\section{Materials and Methods}

\subsection{Materials}

\subsubsection{Antibodies}

Table 1: Antibodies used in this study

Primary antibodies were used at a dilution of 1:1000 and secondary antibodies at 1:10000 for western blotting. A 1:100 dilution was used for flow cytometry. Antibody concentrations for stimulations and affinity purifications can be found in the respective figure legends. (WB-western blotting, FC-flow cytometry, S-stimulation, AP-affinity purification)

\begin{tabular}{|c|c|c|c|}
\hline Antibody & Species (Clone) & Supplier & Application \\
\hline $\begin{array}{l}\alpha \text {-human IgE epsilon } \\
\text { chain }\end{array}$ & Goat (polyclonal) & Abcam & WB, FC,S \\
\hline$\alpha$-human IgE-Biotin & Goat (polyclonal) & Thermo Scientific & AP \\
\hline $\begin{array}{l}\alpha \text {-Goat IgG }(\mathrm{H} \& \mathrm{~L}) \text {-Alexa } \\
\text { Fluor } 647 \mathrm{~F}\left(\mathrm{ab}^{\prime}\right)_{2}\end{array}$ & Donkey (polyclonal) & Abcam & FC \\
\hline $\begin{array}{l}\alpha \text {-human IgM } F\left(a b^{\prime}\right)_{2} \\
\text { fragment }\end{array}$ & Goat (polyclonal) & $\begin{array}{l}\text { Jackson Immuno } \\
\text { research }\end{array}$ & S \\
\hline$\alpha$-human IgM-Biotin & Goat (polyclonal) & Southern Biotech & WB, AP \\
\hline$\alpha$-human IgM-APC & Mouse IgG3 (UHB) & Southern Biotech & FC \\
\hline$\alpha$-human kappa & Goat (polyclonal) & Southern Biotech & WB \\
\hline $\begin{array}{l}\alpha \text {-mouse } \lg G F\left(a b^{\prime}\right)_{2} \\
\text { fragment }\end{array}$ & Goat (polyclonal) & $\begin{array}{l}\text { Jackson Immuno } \\
\text { research }\end{array}$ & $S$ \\
\hline$\alpha$-mouse lgG-CY5 & Goat IgG (polyclonal) & Southern Biotech & FC \\
\hline$\alpha$-mouse $\lambda$-Biotin & Rat IgG2a (RML42) & Biolegend & FC \\
\hline $\begin{array}{l}\text { a-p-p44/42 MAPK } \\
\text { (Erk1/2) (Thr202/Tyr204) }\end{array}$ & Mouse IgG1 (E10) & $\begin{array}{l}\text { Cell Signaling } \\
\text { Technology (CST) }\end{array}$ & WB \\
\hline$\alpha$-pan Erk & Mouse IgG2a (16) & BD Biosciences & WB \\
\hline$\alpha-p-T y r-100$ & Mouse IgG1 (100) & CST & WB \\
\hline$\alpha-p-T y r$ 4G10 & Mouse IgG2a (4G10) & $\begin{array}{l}\text { Upstate } \\
\text { Biotechnology }\end{array}$ & WB \\
\hline$\alpha$-Syk & Mouse IgG2a (4D10) & Santa Cruz & WB \\
\hline$\alpha-p-S y k$ (Tyr323) & Rabbit (polyclonal) & CST & WB \\
\hline$\alpha-P L C \gamma 2$ & Rabbit lgG (polyclonal) & Santa Cruz & WB, AP \\
\hline$\alpha-p-P L C \gamma 2$ (Tyr759) & Rabbit (polyclonal) & CST & WB \\
\hline$\alpha-S L P-65$ & Mouse IgG1 & BAbCo & WB \\
\hline$\alpha-S L P-65$ & Mouse IgG2a (2B11) & BD Biosciences & WB \\
\hline$\alpha-p-B L N K$ (SLP65) & Rabbit (polyclonal) & CST & WB \\
\hline
\end{tabular}




\begin{tabular}{|c|c|c|c|}
\hline (Tyr96) & & & \\
\hline$\alpha$-Grb2 & Mouse IgG1 (3F2) & Millipore & WB \\
\hline$\alpha$-Grap & Goat (polyclonal) & Abnova & WB \\
\hline$\alpha-\beta$ actin & Rabbit IgG (13E5) & CST & WB \\
\hline$\alpha$-GST & Rabbit lgG (polyclonal) & MoBiTec & WB \\
\hline$\alpha-C D 79 A(\lg \alpha)$ & Rabbit (EP3618) & Abcam & WB \\
\hline$\alpha$-CD79A-AlexaFluor647 & Mouse IgG1 (ZL7-4) & BioRad & $\mathrm{FC}$ \\
\hline$\alpha-p-C D 79 A(T y r 182)$ & Rabbit (polyclonal) & CST & WB \\
\hline$\alpha-C D 79 B(\lg \beta)$ & Rabbit (EPR6861) & Abcam & WB \\
\hline$\alpha$-CD79B-AlexaFluor647 & Hamster lgG (HM79-11) & BioRad & $\mathrm{FC}$ \\
\hline$\alpha-C D 19$ & Rabbit (polyclonal) & CST & WB \\
\hline$\alpha$-mouse IgG1 -HRP & Goat IgG (polyclonal) & Southern Biotech & WB \\
\hline$\alpha$-mouse IgG2a-HRP & Goat IgG (polyclonal) & Southern Biotech & WB \\
\hline$\alpha$-mouse lgG2b-HRP & Goat IgG (polyclonal) & Southern Biotech & WB \\
\hline$\alpha$-rabbit IgG-HRP & Goat IgG (polyclonal) & Southern Biotech & WB \\
\hline$\alpha$-goat IgG-HRP & Donkey (polyclonal) & Southern Biotech & WB \\
\hline
\end{tabular}

\subsubsection{Vectors and constructs}

Table 2: Vectors and constructs used in this study

\begin{tabular}{|c|c|c|}
\hline Vector backbone & Insert & Source \\
\hline \multicolumn{3}{|c|}{ Vectors used for cloning or as source of insert } \\
\hline pCRIITOPO & - & Invitrogen \\
\hline pCRIITOPO & hemSvnp-WT & Niklas Engels \\
\hline pCR2.1 & - & Invitrogen \\
\hline pCR2.1 & BNN-Vnpe & This study \\
\hline pUC-18 & $\mathrm{h} \mu \mathrm{HCOC25SG}$ & Niklas Engels \\
\hline pCR2.1 & mGRB2-WT & Lars M. Koenig \\
\hline pCR2.1 & hGRAP-WT & Lars M. Koenig \\
\hline pCR2.1 & $\mathrm{m} \lambda 1 \mathrm{LC}$ & Niklas Engels \\
\hline \multicolumn{3}{|c|}{ Vectors used for retroviral expression of proteins in mammalian cells } \\
\hline pMSCVpuro & - & Clontech \\
\hline pMSCVpuro & hemSvnp-WT & Niklas Engels \\
\hline pMSCVpuro & hemSvnp-YA & Niklas Engels \\
\hline pMSCVpuro & hemSvnp-MVQR & This study \\
\hline pMSCVpuro & hemSvnp-NA & This study \\
\hline pMSCVpuro & $\gamma 2 a m-W T$ & Niklas Engels \\
\hline pMSCVpuro & y2am-YA & Niklas Engels \\
\hline
\end{tabular}




\begin{tabular}{|c|c|c|}
\hline pMSCVpuro & 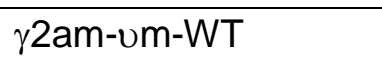 & This study \\
\hline pMSCVpuro & y2am-um-YA & This study \\
\hline pMSCVpuro & huHCvnp & This study \\
\hline pMSCVpuro & huHCvnp/he-Cyt & Niklas Engels \\
\hline pMSCVpuro & huHCvnp/he-TM+Cyt & Niklas Engels \\
\hline MiGRII & - & Elena Vigorito \\
\hline MiGRII & mGRB2-WT & This study \\
\hline MiGRII & mGRB2-W36K & This study \\
\hline MiGRII & mGRB2-R86K & This study \\
\hline MiGRII & mGRB2-W193K & This study \\
\hline MiGRII & hemSvnp-WT & This study \\
\hline MiGRII & hemSvnp-YA & This study \\
\hline MiGRII & huHCvnp & This study \\
\hline MiGRII & $m \lambda 1 L C$ & This study \\
\hline MiRFP & - & Lars M. Koenig \\
\hline MiRFP & hGRAP-WT & This study \\
\hline MiRFP & hGRAP-W36K & This study \\
\hline MiRFP & hGRAP-R86K & This study \\
\hline MiRFP & hGRAP-W195K & This study \\
\hline pCMV & VSV-G & Manfred Juecker \\
\hline \multicolumn{3}{|c|}{ Vectors used for expression of GST fusion proteins in bacteria } \\
\hline pGEX4T-1 & - & GE Healthcare \\
\hline pGEX4T-1 & mGRB2-SH2-WT & Niklas Engels \\
\hline pGEX4T-1 & mGRB2-SH2-R86L & Niklas Engels \\
\hline pGEX4T-1 & hGRAP-SH2-WT & This study \\
\hline pGEX4T-1 & hGRAP-SH2-R86K & This study \\
\hline
\end{tabular}

\subsubsection{Primers}

Table 3: Primers used in this study (Fwd-forward, Rev-reverse)

\begin{tabular}{|l|l|}
\hline Primer name & Primer sequence 5' - 3' \\
\hline M13 Fwd & TGTAAAACGACGGCCAGT \\
\hline M13 Rev & CAGGAAACAGCTATGACC \\
\hline pMSCV Fwd & CCCTTGAACCTCCTCGTTCGACC \\
\hline pMSCV Rev & GAGACGTGCTACTTCCATTTGTC \\
\hline Anolis YA Fwd & CAACAGGCCCTGATGCTAAGAATGTACTCC \\
\hline Anolis YA Rev & GGAGTACATTCTTAGCATCAGGGCCTGTTG \\
\hline hulgE NA Fwd & CTCGACTACACCGCCGTCCTCCAGCCCCAC \\
\hline hulgE NA Rev & GTGGGGCTGGAGGACGGCGGTGTAGTCGAG \\
\hline
\end{tabular}




\begin{tabular}{|l|l|}
\hline hlgE MVQRstop Fwd & CTCCTCATGGTGCAGCGGTAGCTCTCAGCCACG \\
\hline hlgE MVQRstop Rev & CGTGGCTGAGAGCTACCGCTGCACCATGAGGAG \\
\hline GRAP W36K Fwd & GAGGATGACCAGAACAAGTACAAGGCCGAGCTC \\
\hline GRAP W36K Rev & GAGCTCGGCCTTGTACTTGTTCTGGTCATCCTC \\
\hline GRAP R86K Fwd & GAGCCTTCCTGATCAAGGAGAGTGAGAGCTC \\
\hline GRAP R86K Rev & GAGCTCTCACTCTCCTTGATCAGGAAGGCTC \\
\hline GRAP W195K Fwd & GCCCAGACCCCCACAAGTGGCGGGGCCGGTCCTG \\
\hline GRAP W195K Rev & CAGGACCGGCCCCGCCACTTGTGGGGGTCTGGGC \\
\hline VNP BNN Fwd & $\begin{array}{l}\text { ATCGGATCCGCGGCCGCTCTCACCATGGGATGGAGCT } \\
\text { GTATC }\end{array}$ \\
\hline VNP BNN Rev & ATCGCTCGAGACGGTGACCGTGGTCCC \\
\hline
\end{tabular}

\subsubsection{Synthetic peptides}

- Human-e-phospho-ITT peptide

Biotin - RPQTSLD(pY)TNVLQPHA

- Human- $\varepsilon-I T T$ peptide

Biotin - RPQTSLDYTNVLQPHA

$1 \mathrm{mM}$ stock solution was prepared in $1 \mathrm{X}$ PBS. The non-phosphorylated peptide was generated by treating an aliquot of the phospho-peptide with calf intestinal phosphatase for 1 hour at $37^{\circ} \mathrm{C}$. The peptides were purchased from CASLO ApS.

\subsubsection{Mammalian cell lines}

- DG75 (DSMZ ACC 83)

Human Burkitt lymphoma B cell line established from the pleural effusion of a 10year-old boy with Burkitt lymphoma (refractory, terminal) in 1975. The cells were described as EBV (Epstein Barr Virus) negative and express mlgM along with the $\kappa$ light chain (Ben-Bassat et al 1977).

\section{- DG75 EcoBlast (EB)}

DG75 cells were transfected with a vector containing the murine cationic aminoacid transporter 1 (slc7a1) ecotropic receptor for MMLV-based retrovirus docking and blasticidin as a selection marker (Tillmann Brummer, Lars M. Koenig, PhD thesis, 2012).

- DG75GRB2KOEN (referred to as GRB2KO in this study)

DG75 cells deficient for GRB2 were generated using vectors targeting the GRB2 locus by homologous recombination followed by antibiotic selection. The cells were additionally transfected with a vector containing the murine cationic aminoacid transporter 1 (s/c7a1) ecotropic receptor for MMLV-based retrovirus docking and neomycin as a selection marker (Engels et al 2014). 
- DG75GRB2KOENGRAPh\#81 (referred to as GRB2/GRAP dKO in this study) DG75GRB2KOEN cells were targeted with a TALEN construct targeting the GRAP locus generating cells heterozygous for GRAP. The cells have nucleotide deletions in each allele leading to almost undetectable levels of Grap protein expression (Caren Bartsch, PhD thesis, 2016).

- Platinum-E (Plat-E)

Plat-E is a retroviral packaging cell line based on the HEK293T cells. They contain the MMLV (Moloney murine leukemia virus) gag, pol and env genes driven by a strong EF1 $\alpha$ promoter. The presence of an internal ribosome entry site (IRES) site between the gag, pol and env genes and the selection markers (blasticidin and puromycin) ensures high retroviral titers in these cells (Morita et al 2000).

- J558L

$\mathrm{J} 558 \mathrm{~L}$ is a mouse plasmacytoma $\mathrm{B}$ cell line isolated from a BALB/C mouse myeloma. The cells are class switched to $\lg A$ isotype but do not express it, however they secrete a $\lambda$ light chain (Hombach et al 1990). Kindly provided by Michael Reth, Freiburg, Germany.

- Ramos

Human Burkitt lymphoma B cell line originally established from the ascitic fluid of a 3-year-old boy with American-type Burkitt lymphoma in 1972. The cells were described as EBV-negative and carry the $(8 ; 14) \operatorname{lgH} ; \mathrm{MYC}$ translocation and TP53 mutations. They express slgM along with the $\lambda$ light chain (Klein et al 1975). Kindly provided by Michael Reth, Freiburg, Germany.

- Ramos HL KO \#39

Ramos cells deficient for the Ig heavy chain $(H)$ and the Ig lambda light chain (L). Kindly provided by Michael Reth, Freiburg, Germany.

- Ramos Mb1 KO

Ramos cells deficient for the Mb1 gene encoding for $\lg \alpha$. Kindly provided by Michael Reth, Freiburg, Germany.

\subsubsection{Cell culture media for mammalian cell lines}

- R10 medium

RPMI 1640 + Glutamax, 10\% heat inactivated fetal calf serum (FCS), $1 \%$ penicillin/streptomycin, $50 \mu \mathrm{m} \beta$-mercaptoethanol, $1 \mathrm{mM}$ sodium pyruvate

- R0 medium

R10 without FCS

- Freezing medium $90 \%$ heat inactivated FCS, $10 \%$ DMSO

- Selection markers

$3 \mu \mathrm{g} / \mathrm{ml}$ puromycin or $10 \mu \mathrm{g} / \mathrm{ml}$ blasticidin 
- "Heavy" SILAC medium for mass spectrometry

SILAC RPMI 1640 Medium, 10\% dialyzed fetal bovine serum (PAN Biotech), 1\% penicillin/streptomycin, $0.12 \mathrm{mM} \mathrm{L-Arginine}\left({ }^{13} \mathrm{C}_{6}\right), 0.27 \mathrm{mM}$ L-Lysine $\left(\mathrm{D}_{4}\right)$

- "Light" SILAC medium for mass spectrometry

SILAC RPMI 1640 Medium, 10\% dialyzed fetal bovine serum (PAN Biotech), 1\% penicillin/streptomycin

\subsubsection{Bacterial strains}

- $\quad$ TOP10F' - used for cloning Genotype - F'\{laclq Tn10 (TetR)\} mcrA $\Delta$ (mrr-hsdRMS-mcrBC) Ф80lacZ $\Delta$ M15 $\Delta$ lacX74 recA1 araD139 $\Delta$ (ara-leu)7697 gaN gaK rpsL endA1 nupG

- BL21 DE3 - used for protein expression fhuA2 [lon] ompT gal $(\lambda$ DE3) [dcm] $\Delta$ hsdS $\lambda$ DE3 $=\lambda$ sBamHlo $\Delta$ EcoRI-B int::(lacl::PlacUV5::T7 gene1) i21 $\Delta$ nin5

\subsubsection{Media and plates for bacteria}

- Luria Bertani (LB) medium

$10 \mathrm{~g} / \mathrm{L}$ Tryptone, $5 \mathrm{~g} / \mathrm{L}$ yeast extract, $5 \mathrm{~g} / \mathrm{L} \mathrm{NaCl}, \mathrm{pH}$ 7.0. Autoclaved at $121^{\circ} \mathrm{C}$, 1.25 bar for $30 \mathrm{~min}$.

- 2X Yeast Tryptone (2YT) medium

$16 \mathrm{~g} / \mathrm{L}$ Tryptone, $10 \mathrm{~g} / \mathrm{L}$ yeast extract, $10 \mathrm{~g} / \mathrm{L} \mathrm{NaCl}, \mathrm{pH}$ 7.0. Autoclaved at $121^{\circ} \mathrm{C}$, 1.25 bar for $30 \mathrm{~min}$.

- Agar plates

LB medium, $2 \%(w / v)$ agar

- Selection markers

$100 \mu \mathrm{g} / \mathrm{ml}$ ampicillin

\subsubsection{Chemical reagents}

All chemicals and reagents used in this work were purchased from Carl Roth, Sigma, Merck or Invitrogen unless categorically specified.

\subsubsection{Buffers and solutions}

Table 4: Buffers and solutions used in this study

\begin{tabular}{|l|l|}
\hline Buffer/solution & Composition \\
\hline B cell lysis buffer & $137.5 \mathrm{mM} \mathrm{NaCl}, 50 \mathrm{mM}$ Tris pH 7.8, $1 \mathrm{mM}$ \\
& $\mathrm{Na}_{3} \mathrm{VO}_{4}, 0.5 \mathrm{mM}$ EDTA pH 8.0, 10\% \\
\hline
\end{tabular}




\begin{tabular}{|c|c|}
\hline & $\begin{array}{l}\text { glycerol, } 1 \mathrm{X} \text { protease inhibitor cocktail, } 1 \% \\
\text { NP-40 detergent, add. } \mathrm{ddH}_{2} \mathrm{O}\end{array}$ \\
\hline 1X Phosphate buffer saline (PBS) & $\begin{array}{l}137 \mathrm{mM} \mathrm{NaCl}, 27 \mathrm{mM} \mathrm{KCl}, 8.6 \mathrm{mM} \\
\mathrm{Na}_{2} \mathrm{HPO}_{4} .12 \mathrm{H}_{2} \mathrm{O}, 1.4 \mathrm{mM} \mathrm{KH}_{2} \mathrm{PO}_{4} \text {, add. } \\
\text { dd }_{2} \mathrm{O}\end{array}$ \\
\hline $1 \mathrm{X}$ Krebs ringer solution & $\begin{array}{l}140 \mathrm{mM} \mathrm{NaCl}, 4 \mathrm{mM} \mathrm{KCl}, 10 \mathrm{mM} \text { D- } \\
\text { glucose, } 10 \mathrm{mM} \text { HEPES pH 7.4, } 1 \text { mM } \\
\mathrm{CaCl}_{2}\end{array}$ \\
\hline $1 \mathrm{X}$ Tris buffer saline with tween (TBST) & $\begin{array}{l}20 \mathrm{mM} \text { Tris } \mathrm{pH} 7.6,137 \mathrm{mM} \mathrm{NaCl}, 0.1 \% \\
\text { tween-20, add. } \text { dd }_{2} \mathrm{O}\end{array}$ \\
\hline $\begin{array}{l}\text { Enhanced chemiluminiscence (ECL) } \\
\text { solution A }\end{array}$ & $200 \mathrm{ml} \mathrm{0.1} \mathrm{M} \mathrm{Tris} \mathrm{pH} \mathrm{8.6,} 50$ mg luminol \\
\hline ECL solution $B$ & $\begin{array}{l}50 \mathrm{ml} \text { DMSO, } 55 \mathrm{mg} \text { para-hydroxycoumaric } \\
\text { acid }\end{array}$ \\
\hline 2X Laemlli buffer & $\begin{array}{l}62.5 \mathrm{mM} \text { TrisHCL pH 6.8, 2\% SDS, } 20 \% \\
\text { glycerol, } 5 \% \beta \text {-mercaptoethanol, } 0.025 \% \\
\text { bromophenolblue, add. dd } \mathrm{H}_{2} \mathrm{O}\end{array}$ \\
\hline Coomassie staining solution & $\begin{array}{l}0.25 \% \text { Coomassie Brilliant Blue R-250, } \\
45 \% \text { methanol, } 10 \% \text { acetic acid, add. } \\
\mathrm{ddH}_{2} \mathrm{O}\end{array}$ \\
\hline 10\% Resolving gel SDS-PAGE & $\begin{array}{l}375 \mathrm{mM} \text { Tris } \mathrm{pH} 8.8,0.1 \% \text { SDS, } 10 \% \\
\text { Acrylamide and bisacrylamide, } 0.00065 \% \\
\text { APS, } 0.001 \% \text { TEMED, add. } \mathrm{dd}_{2} \mathrm{O}\end{array}$ \\
\hline $5 \%$ Stacking gel SDS-PAGE & $\begin{array}{l}125 \mathrm{mM} \text { Tris pH6.8, } 0.1 \% \text { SDS, } 5 \% \\
\text { Acrylamide and bisacrylamide, } 0.001 \% \\
\text { APS, } 0.001 \% \text { TEMED, add. } \mathrm{ddH}_{2} \mathrm{O}\end{array}$ \\
\hline 1X SDS-PAGE running buffer & $\begin{array}{l}25 \mathrm{mM} \text { Tris, } 192 \mathrm{mM} \text { glycine, } 0.1 \% \text { (w/v) } \\
\text { SDS, add. } \mathrm{ddH}_{2} \mathrm{O}\end{array}$ \\
\hline 1X Blotting buffer & $\begin{array}{l}39 \mathrm{mM} \text { Glycine, } 48 \mathrm{mM} \text { Tris, } 0.0375 \%(\mathrm{w} / \mathrm{v}) \\
\mathrm{SDS}, 0.01 \%(\mathrm{w} / \mathrm{v}) \mathrm{NaN} 3,20 \%(\mathrm{v} / \mathrm{v}) \\
\text { methanol, add. } \mathrm{ddH}_{2} \mathrm{O}\end{array}$ \\
\hline Blocking solution & $5 \%$ BSA, $0.001 \% \mathrm{NaN}_{3}$, add. 1 X TBST \\
\hline $1 \mathrm{X}$ Tris acetate EDTA (TAE) & $\begin{array}{l}40 \text { mM Tris-acetate } \mathrm{pH} 8.0,10 \mathrm{mM} \mathrm{NaOAc} \text {, } \\
1 \text { mM EDTA, add. } \mathrm{ddH}_{2} \mathrm{O}\end{array}$ \\
\hline
\end{tabular}

\subsubsection{Enzymes and their supplier}

- Restriction endonucleases

- T4 DNA Ligase

- Calf intestinal phosphatase
New England Biolabs

New England Biolabs

New England Biolabs 
- Pfu polymerase

- Phusion polymerase
Promega

New England Biolabs

\subsubsection{Additional materials and their supplier}

- 5X Phusion Buffer

- 6X DNA loading dye

- 10X NEBuffers 2.1/3.1/Cutsmart

- 10X BSA

- 10X Pfu Buffer

- 10X T4 Buffer

- Blasticidin

- Bay61-3606 inhibitor

- Cell culture material

- GeneRuler ${ }^{\mathrm{TM}} 1$ kb DNA ladder

- Glutathione-Sepharose beads

- Indo-1 AM

- Nitrocellulose filter Hybond ECL ${ }^{\mathrm{TM}}$

- Whatman Gel Blotting Paper

- Puromycin

- Polybrene

- L-Arginine: $\mathrm{HCL}\left({ }^{13} \mathrm{C}_{6}\right)$

- L-Lysine:2HCL $\left(4,4,5,5-\mathrm{D}_{4}\right)$

- NP-40 (IGEPAL CA-630)

- NuPAGE LDS sample buffer (4X)

- NuPAGE sample reducing buffer (10X)

- PP1 inhibitor

- Prestained broad range protein marker (6.5-175 kDa)

- Prestained broad range protein marker (11-190 kDa)

- Protein AG-Agarose beads

- RPMI 1640 + Glutamax

- SILAC RPMI 1640 Medium

- Streptavidin-Sepharose beads

- Streptavidin APC

- Streptavidin HRP

- TransIT® 293 Transfection Reagent
New England Biolabs

Thermo Fischer Scientific

New England Biolabs

New England Biolabs

Promega

New England Biolabs

InvivoGen

Calbiochem

Greiner, Sarstedt, Nunc

Thermo Fischer Scientific

GE Healthcare

Invitrogen

GE Healthcare

GE Healthcare

InvivoGen

Sigma

Cambridge Isotope Laboratories

Cambridge Isotope Laboratories

Sigma-Aldrich

Invitrogen

Invitrogen

Calbiochem

New England Biolabs

New England Biolabs

Santa Cruz Biotechnology

Gibco

Thermo Scientific

GE Healthcare

BD Biosciences

Southern Biotech

Mirus 


\subsubsection{Instruments and their supplier}

- LSRII

- FACS Calibur

- ChemoCam Imager

- NanoDrop 2000

\subsubsection{Softwares and their source}

- Clone Manager

- FlowJo 7.6.5

- CellQuest Pro

- BD FACSDIVA

- Office for Mac 2011

- Photoshop CS4

- Corel Draw X5

- Image J

- Perseus 1.5.5.0

- $\mathrm{NCBI}$ database

- Clustal Omega alignment tool

- Uniprot protein database
Becton Dickinson

Becton Dickinson

Intas

Thermo Scientific
SECentral

TreeStar

Becton Dickinson

Becton Dickinson

Microsoft

Adobe

Corel Corporation

National Institute of Health

Cox and Mann, 2008

NCBI

EMBL-EBI

Uniprot Consortium

\subsection{Methods}

\subsubsection{Culturing mammalian cells}

All cell lines were maintained in R10 medium under optimal growth conditions of $37^{\circ} \mathrm{C}$ and $5 \% \mathrm{CO}_{2}$. The $\mathrm{B}$ cell lines were passaged at a dilution of $1: 5$ for daily use. The adherent Plate-E cells were passaged by removing the medium, washing with $1 \mathrm{ml} 1 \mathrm{X}$ PBS and incubating with $1 \mathrm{ml}$ Trypsin-EDTA at $37^{\circ} \mathrm{C}$ for $5 \mathrm{~min}$. Then, $9 \mathrm{ml}$ fresh R10 medium was added and the required number of Plat-E cells were seeded onto a new dish.

\subsubsection{Freezing and thawing of mammalian cells}

To freeze cells, about 5 million cells in R10 medium were harvested by centrifugation at $300 \mathrm{~g}$ for $5 \mathrm{~min}$ at room temperature (RT) and the supernatant was discarded. The pellet was resuspended in $1 \mathrm{ml}$ freezing medium, transferred to a vial and kept on ice. Short term storage of cells was done at $-80^{\circ} \mathrm{C}$ and long term storage at $-140^{\circ} \mathrm{C}$. Frozen vials were thawed in a $37^{\circ} \mathrm{C}$ water bath, the culture was added to $10 \mathrm{ml} \mathrm{R} 10$ medium and the cells 
were harvested at $300 \mathrm{~g}, \mathrm{RT}, 5 \mathrm{~min}$. The pellet was resuspended in $10 \mathrm{ml}$ fresh $\mathrm{R} 10$ medium and seeded onto a $10 \mathrm{~cm}$ dish. The culture was allowed to grow to a confluency of about $90 \%$ before use.

\subsubsection{Restriction enzyme digestion of DNA for cloning}

1-3 $\mu \mathrm{g}$ of DNA was incubated with $0.5-1.0 \mu \mathrm{l}$ restriction enzyme and the respective NEBuffer in a total volume of $20 \mu \mathrm{l}$ adjusted with $\mathrm{ddH}_{2} \mathrm{O}$ at $37^{\circ} \mathrm{C}$ for 3 hours or $\mathrm{O} / \mathrm{N}$. Vector preparations were treated with $1.5 \mu \mathrm{l} \mathrm{CIP}$ for an additional $60 \mathrm{~min}$ to prevent vector re-ligation. The samples were then mixed with $1 \mathrm{X}$ DNA loading dye and run on an agarose gel and visualized using the Gel Imager.

\subsubsection{Agarose gel electrophoresis and purification of DNA fragments}

Size based separation of DNA fragments was done using agarose gel electrophoresis. Depending on the size of the DNA fragment to be separated, a range of $1-2 \%$ agarose gels containing $0.1 \%(\mathrm{v} / \mathrm{v})$ Ethidium bromide were cast and arranged in a chamber containing 1X TAE running buffer. The DNA samples were mixed with 1X DNA loading dye and loaded alongside $10 \mu$ of the $1 \mathrm{~Kb}$ DNA ladder. Electrophoresis was performed at $1.2 \mathrm{~V} / \mathrm{cm}^{2}$ and the DNA bands were visualized under ultraviolet light using the Gel Imager. The required DNA bands were excised from the agarose gel and purified using the Promega Wizard® SV Gel and PCR Clean-Up System as per manufacturer's protocol. The DNA was normally eluted in $20 \mu \mathrm{ldd} \mathrm{H}_{2} \mathrm{O}$.

\subsubsection{Ligation of DNA fragments}

Ligation of inserts into the preferred vectors was done by mixing $3 \mu \mathrm{g}$ Insert, $1 \mu \mathrm{g}$ vector, $1 \mu \mathrm{l}$ T4 DNA ligase enzyme and $1 \mu \mathrm{l}$ 10X T4 ligase buffer. The final reaction volume was adjusted to $10 \mu \mathrm{l}$ with $\mathrm{ddH}_{2} \mathrm{O}$ and the ligation performed at $\mathrm{RT}$ for 1 hour or at $14^{\circ} \mathrm{C} \mathrm{O} / \mathrm{N} .1$ $\mu \mathrm{l}$ of the ligation mix was transformed into competent bacterial cells and plated on LB agar plates containing $100 \mu \mathrm{g} / \mathrm{ml}$ ampicillin (LB-Amp).

\subsubsection{Transformation of plasmid DNA into competent bacterial cells}

TOP10F' and BL21 bacterial strains were made competent using the $\mathrm{CaCl}_{2}$ method described in Inoue et al 1990 and stored at $-20^{\circ} \mathrm{C}$. A $50 \mu$ aliquot of competent cells was thawed and incubated with $1 \mu \mathrm{l}$ of the ligation reaction or $50 \mathrm{ng}$ plasmid DNA for $20 \mathrm{~min}$ on ice. After heat shock at $42^{\circ} \mathrm{C}$ for $42 \mathrm{sec}$, the cells were immediately put on ice for 2 min. The cells were then directly plated onto a LB-Amp agar plate and incubated $\mathrm{O} / \mathrm{N}$ at $37^{\circ} \mathrm{C}$ for colonies to grow. Alternatively, $5 \mu$ of the transformation mix containing a 
previously sequenced clone was used to directly inoculate $6 \mathrm{ml}$ liquid LB-Amp to grow an $\mathrm{O} / \mathrm{N}$ culture for plasmid purification.

\subsubsection{Mini- and midi-preparations of plasmid DNA}

For small scale (mini) plasmid DNA preparation, $6 \mathrm{ml}$ LB-Amp was inoculated with a colony and grown $\mathrm{O} / \mathrm{N}$ at $37^{\circ} \mathrm{C}$ and $200 \mathrm{rpm}$ agitation speed. For large scale (midi) preparations $50 \mathrm{ml} \mathrm{O} / \mathrm{N}$ cultures were used. The cultures were then used for plasmid DNA purification using the Qiagen QIAprep ${ }^{\circledR}$ Spin Miniprep kit or Qiagen HiSpeed Plasmid Midi kit as per the manufacturer's protocol and DNA eluted in $30 \mu \mathrm{l}$ or $100 \mu \mathrm{l}$ $\mathrm{dd}_{2} \mathrm{O}$ respectively.

\subsubsection{Analyzing DNA quality and sequence}

$1 \mu$ DNA was used to measure optical density at $260 \mathrm{~nm}$ at the NanoDrop 2000 (Thermo scientific) and an optical density of 1.0 was quantified as $50 \mu \mathrm{g} / \mathrm{ml}$ DNA. A sequencing reaction was setup by taking $1200 \mathrm{ng}$ of purified DNA, $3 \mu \mathrm{M}$ primer and the volume adjusted to $15 \mu \mathrm{l}$ with $\mathrm{dd}_{2} \mathrm{O}$. Sequencing was done by SEQLAB Sequence Laboratories Goettingen $\mathrm{GmbH}$. All DNA preparations were stored at $4^{\circ} \mathrm{C}$ for short periods and at $20^{\circ} \mathrm{C}$ for long term storage.

\subsubsection{Polymerase chain reaction (PCR)}

The master mix was prepared as described below and split into two $25 \mu \mathrm{l}$ reactions for the PCR. One containing $1 \mu \mathrm{l}$ Phusion polymerase and the other without Phusion polymerase to serve as negative control. The PCR products were run on an agarose gel and further purified as described before.

\section{Master mix}

$\begin{array}{ll}\text { Ingredient } & \text { Amount } \\ \text { dd } \mathrm{H}_{2} \mathrm{O} & 36 \mu \mathrm{l} \\ 5 \mathrm{X} \text { Phusion buffer } & 10 \mu \mathrm{l} \\ 10 \mathrm{mM} \text { dNTPs } & 1 \mu \mathrm{l} \\ 100 \mathrm{ng} / \mu \mathrm{l} \mathrm{Template} \mathrm{DNA} & 1 \mu \mathrm{l} \\ 10 \mu \mathrm{M} \text { Primer Fwd } & 1 \mu \mathrm{l} \\ 10 \mu \mathrm{M} \text { Primer Rev } & 1 \mu \mathrm{l} \\ \text { Total } & 50 \mu \mathrm{l}\end{array}$




\section{PCR cycle}

Temperature

Time

$94^{\circ} \mathrm{C}$

$2 \min$

$94^{\circ} \mathrm{C}$

$30 \mathrm{sec}$

$55^{\circ} \mathrm{C}$

$1 \mathrm{~min}$

$68^{\circ} \mathrm{C}$

$30 \mathrm{sec}$ per $1 \mathrm{~kb}$ plasmid

$68^{\circ} \mathrm{C}$

4 min final extension

$4^{\circ} \mathrm{C}$

End

\subsubsection{Site directed mutagenesis}

For introduction of specific mutations in the DNA sequence, amplification of the entire plasmid DNA was done using complementary primers that contained the required mutation. The master mix was prepared as described below and split into two $50 \mu \mathrm{l}$ reactions for the PCR. One containing $1 \mu \mathrm{l}$ Pfu polymerase and the other without Pfu polymerase to serve as negative control.

\section{Master mix}

$\begin{array}{ll}\text { Ingredient } & \text { Amount } \\ \mathrm{dd}_{2} \mathrm{O} & 82 \mu \mathrm{l} \\ 10 \mathrm{X} \text { Pfu Buffer } & 10 \mu \mathrm{l} \\ 10 \mathrm{mM} \text { dNTPs } & 2 \mu \mathrm{l} \\ 100 \mathrm{ng} / \mu \mathrm{l} \text { Template DNA } & 2 \mu \mathrm{l} \\ 10 \mu \mathrm{M} \text { Primer Fwd } & 2 \mu \mathrm{l} \\ 10 \mu \mathrm{M} \text { Primer Rev } & 2 \mu \mathrm{l} \\ \text { Total } & 100 \mu \mathrm{l}\end{array}$

\section{PCR cycle}

Temperature

Time

$94^{\circ} \mathrm{C}$

$2 \min$

$94^{\circ} \mathrm{C}$

$30 \mathrm{sec}$

$55^{\circ} \mathrm{C}$

$1 \mathrm{~min}$

$68^{\circ} \mathrm{C}$

2 min per $1 \mathrm{~kb}$ plasmid

$68^{\circ} \mathrm{C}$

10 min final extension

$4^{\circ} \mathrm{C}$

End

The reactions were then further divided into two aliquots of $25 \mu$ l each. One was loaded on an agarose gel for visualization and the other was incubated with $1 \mu \mathrm{Ipnl}$ at $37^{\circ} \mathrm{C}$ for 1 hour. The Dpnl cleaves the template plasmid which is methylated and leaves the PCR product intact. The product was then used directly for transformation into competent bacteria for further purification followed by quality and sequence tests. 


\subsubsection{TA cloning}

PCR products were cloned into the pCR2.1 vector using the TA cloning ${ }^{\circledR}$ kit from Invitrogen. To add 3' deoxyadenosine overhangs, $20 \mu$ of the purified PCR product was mixed with $2 \mu \mathrm{l}$ 10X Taq polymerase buffer, $1 \mu \mathrm{l}$ dATPs, $1 \mu \mathrm{l}$ Taq polymerase and incubated at $70^{\circ} \mathrm{C}$ for $25 \mathrm{~min}$. The product was then ligated as described in section 5.2.5 with the pCR2.1 vector containing 3' deoxythymidine overhangs. Agar plates containing $50 \mu \mathrm{l} \mathrm{X-gal}(50 \mathrm{mg} / \mathrm{ml}$ stock) and $50 \mu \mathrm{l}$ IPTG $(0.1 \mathrm{M}$ stock) were plated with $1 \mu \mathrm{l}$ of the ligation product for examining successful ligation by blue-white screening.

\subsubsection{Retroviral transduction of B cells}

Retroviral transduction was done using the ecotropic Plat-E retroviral packaging cell line which produces viruses carrying the DNA of interest. Viral particles produced by the PlatE's were used to infect $B$ cells that carried the murine ecotropic receptor for viral particle recognition. For cells that did not carry the ecotropic receptor, the transfection solution was mixed with the packaging vector PCMV-VSV-G which enables infection of human cells via the vesicular stomatis virus-glycoprotein. To this end, the adherent Plat-E cells were grown to about $60 \%$ confluence on a $6 \mathrm{~cm}$ dish. The medium was carefully replaced with $3 \mathrm{ml}$ fresh R10 (pre-warmed to $37^{\circ} \mathrm{C}$ ), the transfection solution added and the culture grown at $37^{\circ} \mathrm{C} \mathrm{O} / \mathrm{N}$. The transfection solution was prepared as follows:

- $250 \mu \mathrm{R} 0$ at $37^{\circ} \mathrm{C}$

- Add $8 \mu$ TransIT, mix well and incubate at RT for 15 min

- Add $3 \mu \mathrm{g}$ DNA, mix well and incubate at RT for $20 \mathrm{~min}(0.5 \mu \mathrm{g}$ pCMV-VSV-G included if required)

The next day, $3 \mathrm{ml}$ fresh R10 (pre-warmed to $37^{\circ} \mathrm{C}$ ) was added and the culture grown at $37^{\circ} \mathrm{C} \mathrm{O} / \mathrm{N}$. The supernatant was carefully collected after centrifugation and the Plat-E cells pelleted by centrifugation at $300 \mathrm{~g}$ at RT for $5 \mathrm{~min}$. About $4 \mathrm{ml}$ of the supernatant was used to infect about 2 million B cells along with $4 \mu \mathrm{g} / \mathrm{ml}$ Polybrene solution. The cells were grown at $37^{\circ} \mathrm{C}$ and to obtain a nearly confluent $10 \mathrm{~cm}$ dish. The cells were then put under selection with $3 \mu \mathrm{g} / \mathrm{ml}$ puromycin. After at least 7 days of selection, the efficiency of infection was analyzed using flow cytometry for cell surface proteins. Alternatively, cells that did not carry selection markers or underwent incomplete selection were sorted via fluorescent activated cell sorting (FACS).

\subsubsection{Cell sorting}

Cells were sorted by FACS at the Cell Sorting Facility of the University Medical Center Goettingen with the technical assistance of Sabrina Becker. Approximately 5-7 million cells were washed and stained with fluorescently labeled antibodies against surface proteins whereas GFP/RFP positive cells were simply washed and finally resuspended in 
$500 \mu \mathrm{l} 1 \mathrm{X}$ PBS for cell sorting. Sorted cells were collected in R10 medium, washed twice in R10 medium before resuspension in $200 \mu \mathrm{l} 10$ in 96 well round bottom plates. Cells were grown at $37^{\circ} \mathrm{C}$ and slowly expanded for use and storage.

\subsubsection{Surface staining and protein expression analysis via flow cytometry}

Surface expression of proteins was measured using fluorescently labeled antibodies against the protein of interest. 1 million cells were harvested at $300 \mathrm{~g}, 4^{\circ} \mathrm{C}$ for $5 \mathrm{~min}$ and washed once in 1X PBS. The cell pellet was dissolved in $100 \mu \mathrm{l}$ X PBS and $1 \mu \mathrm{l}$ of the fluorescently labeled antibody was added to the solution, vortexed and incubated on ice for 10 min protected from light. The cells were washed with $1 \mathrm{X}$ PBS and the pellet dissolved in $300 \mu \mathrm{l}$ 1X PBS for measurement at the FACS calibur or BD LSRII. Stained untransfected cells or unstained cells were used as negative controls to measure background fluorescence. Cells expressing GFP and/or RFP were washed once in $1 \mathrm{X}$ PBS and resuspended in $300 \mu \mathrm{l} 1 \mathrm{X}$ PBS for measurement. Data was acquired using the CellQuestPro or FACSDIVA software's and data was processed with FlowJo and Microsoft PowerPoint.

\subsubsection{Preparation of B cell lysates}

B cells were grown to about $70 \%$ confluency and harvested by centrifugation at $300 \mathrm{~g}$, RT for $5 \mathrm{~min}$. The cell pellet was resuspended in 1X PBS and the cells were counted using a hemocytometer. The cells were harvested, resuspended in $10 \mathrm{ml} \mathrm{R0}$ and starved for 30 min at $37^{\circ} \mathrm{C}$ with regular mixing. The cells were harvested again and resuspended in 200 $\mu \mathrm{l}$ R0 per 5 million cells and stimulated with the appropriate anti-BCR antibody for the stipulated time points or left untreated in the case of unstimulated samples. The cell pellet was harvested in the minicentrifuge at $2000 \mathrm{~g}$ for $10 \mathrm{sec}$, the media removed completely and the cells lysed in $20 \mu \mathrm{l}$ B cell lysis buffer (1\% NP40 detergent) per 1 million cells. Lysis was performed on ice for $10 \mathrm{~min}$ and the cell debris pelleted at $20000 \mathrm{~g}, 4^{\circ} \mathrm{C}$ for 10 min. The supernatant comprising the cell lysate was separated and mixed 1:1 with $2 \mathrm{X}$ laemlli buffer, boiled at $95^{\circ} \mathrm{C}$ for $5 \mathrm{~min}$ and $40 \mu \mathrm{l}$ ( 1 million cell lysates) sample was analyzed by SDS-PAGE and immunoblotting. Lysates boiled in laemlli were stored at $20^{\circ} \mathrm{C}$ for long term storage and boiled again prior to use.

\subsubsection{SDS-PAGE}

Size based separation of proteins was done using standard SDS-PAGE. A $10 \%$ polyacrylamide resolving gel and $5 \%$ polyacrylamide stacking gel was used. The gels were cast and run in 1X SDS running buffer using the Hoefer SE600 Ruby system by GE Healthcare. Samples were prepared by boiling in laemlli buffer at $95^{\circ} \mathrm{C}$ for $5 \mathrm{~min}$ and 
loaded onto the gel alongside a protein ladder. The separating gel was run at $25 \mathrm{~mA}, 250$ $\mathrm{V}$ and the resolving gel at $35 \mathrm{~mA}, 250 \mathrm{~V}$. For the detection of GST fusion proteins, the mini gel system from BioRad was utilized with the same gel composition as mentioned above. The mini gel was run at $15 \mathrm{~mA}, 250 \mathrm{~V}$ for the separating gel and $20 \mathrm{~mA}, 250 \mathrm{~V}$ for the resolving gel.

\subsubsection{Immunoblotting / western blotting}

The detection of specific proteins that were separated by SDS-PAGE was carried out by immunoblotting and detection via ECL. The blotting chamber was placed with the anode at the bottom followed by a layer of whatman paper, nitrocellulose membrane, the gel, and another layer of whatman paper, all pre-soaked in blotting buffer. The excess liquid along with the air bubbles carefully removed by applying pressure with a rolling glass rod. The cathode was placed on top and protein transfer carried out at $16 \mathrm{~V}, 240 \mathrm{~mA}$ for 70 min. The membrane was then washed in 1X TBST (used as wash buffer) and blocked at room temperature for 60 min with blocking solution. The membrane was washed again and incubated $\mathrm{O} / \mathrm{N}$ with the primary antibody. The membrane was then washed for $5 \mathrm{~min}$, incubated with Horse radish peroxidase (HRP) labeled secondary antibody for 60 min and washed thrice for 15 min each before ECL-mediated detection. An ECL solution was prepared with $4 \mathrm{ml}$ ECL solution $\mathrm{A}, 1.2 \mu \mathrm{l} \mathrm{H}_{2} \mathrm{O}_{2}$ and $400 \mu \mathrm{l} \mathrm{ECL}$ solution $\mathrm{B}$ and poured over the membrane. The light generated by excitation of oxidized luminol by the HRP was detected using the ChemoCam Imager, Intas. The blot images were processed in Photoshop CS4 and CorelDraw and blot quantification was done using ImageJ and Microsoft Excel. The quantified values were normalized by setting the highest value to 1.0.

\subsubsection{Affinity purification using antibodies or biotinylated peptides}

B cells were grown to about $70 \%$ confluency and harvested at $300 \mathrm{~g}$, RT for $5 \mathrm{~min}$. The cell pellet was resuspended in 1X PBS and the cells were counted. The cells were harvested, resuspended in $10 \mathrm{ml} \mathrm{R0}$ and starved for $30 \mathrm{~min}$ at $37^{\circ} \mathrm{C}$ with regular mixing. 30 million cells per sample were resuspended in $500 \mu \mathrm{R} 0$ and stimulated with anti-BCR antibodies for the stipulated time points or left unstimulated. The cells were harvested in the minicentrifuge at $2000 \mathrm{~g}$ for $10 \mathrm{sec}$, washed once with 1X PBS and then lysed with $600 \mu \mathrm{l}$ lysis buffer on ice for $10 \mathrm{~min}$. The cell debris were pelleted at $20000 \mathrm{~g}, 10 \mathrm{~min}, 4^{\circ} \mathrm{C}$ and the cell lysates were incubated with $2 \mu \mathrm{g}$ of the purifying antibody and $30 \mu \mathrm{l}$ Protein AG-Agarose beads for 2 hours at $4^{\circ} \mathrm{C}$ with constant rotation. The beads were then washed thrice with lysis buffer at $500 \mathrm{~g}, 4 \mathrm{~min}, 4^{\circ} \mathrm{C}$. The beads were mixed with $30 \mu \mathrm{l} 2 \mathrm{X}$ laemlli and boiled at $95^{\circ} \mathrm{C}$ for $5 \mathrm{~min}$. The samples were analyzed by SDS-PAGE and immunoblotting. In case of purification of proteins that associate with the phospho-lgEITT, the cell lysates from anti-human-IgE stimulated DG75 B cells were incubated with 1 $\mu \mathrm{M}$ of the biotinylated peptide and $30 \mu \mathrm{l}$ streptavidin beads for 2 hours at $4^{\circ} \mathrm{C}$ with 
constant rotation. The beads were then washed thrice with lysis buffer at $500 \mathrm{~g}, 4 \mathrm{~min}$, $4^{\circ} \mathrm{C}$. The beads were mixed with $30 \mu \mathrm{l} 2 \mathrm{X}$ laemlli and boiled at $95^{\circ} \mathrm{C}$ for $5 \mathrm{~min}$. The samples were analyzed by SDS-PAGE and immunoblotting.

\subsubsection{BCR purification}

B cells were grown to about $70 \%$ confluency and harvested at $300 \mathrm{~g}$, RT for $5 \mathrm{~min}$. The cell pellet was resuspended in 1X PBS and the cells counted. The cells were harvested, resuspended in $20 \mathrm{ml} \mathrm{R0}$ and starved for $30 \mathrm{~min}$ at $37^{\circ} \mathrm{C}$ with regular mixing. In Fig. 4, 70 million cells per sample were resuspended in $1 \mathrm{ml} \mathrm{R0}$ and either left untreated or treated with $30 \mu \mathrm{M}$ PP1 or $30 \mu \mathrm{M}$ Bay61-3606 for $30 \mathrm{~min}$. They were then stimulated with 10 $\mu \mathrm{g} / \mathrm{ml}$ anti-human-lgE-Biotin antibody for $3 \mathrm{~min}$ or left unstimulated at $37^{\circ} \mathrm{C}$. The cells were harvested in the minicentrifuge, washed once with 1X PBS and then lysed with 600 $\mu \mathrm{l}$ lysis buffer on ice for $10 \mathrm{~min}$. $10 \mu \mathrm{g} / \mathrm{ml}$ anti-human-lgE antibody was added to the unstimulated samples after cell lysis. For purification of surface BCRs in Fig. 18, 30 million cells were treated with $10 \mu \mathrm{g} / \mathrm{ml}$ anti-human-IgE-Biotin antibody or $20 \mu \mathrm{g} / \mathrm{ml}$ anti-humanIgM-Biotin on ice for $5 \mathrm{~min}$. The cells were harvested in the minicentrifuge, washed thoroughly twice with 1 X PBS and then lysed with $600 \mu$ lysis buffer on ice for $10 \mathrm{~min}$. In each case, the cell debris were pelleted at $20000 \mathrm{~g}, 10 \mathrm{~min}, 4^{\circ} \mathrm{C}$ and the cell lysates were incubated with $30 \mu \mathrm{l}$ streptavidin beads for 2 hours at $4^{\circ} \mathrm{C}$ with constant rotation. The beads were then washed thrice with lysis buffer at $500 \mathrm{~g}, 4 \mathrm{~min}, 4^{\circ} \mathrm{C}$. The beads were mixed with $30 \mu \mathrm{l} 2 \mathrm{X}$ laemlli and boiled at $95^{\circ} \mathrm{C}$ for $5 \mathrm{~min}$. The samples were analyzed by SDS-PAGE and immunoblotting.

\subsubsection{Isolation of GST fusion proteins}

BL21 cells were transformed with the pGEX4T-1 vector containing the Grb2 and Grap $\mathrm{SH} 2$ domains. Glycerol stocks were prepared by mixing glycerol and a O/N culture in 1:1 ratio and kept at $-80^{\circ} \mathrm{C}$ for long term storage. The glycerol stock was thawed and $1 \mu \mathrm{l}$ each was used to inoculate two $6 \mathrm{ml}$ cultures grown O/N at $37^{\circ} \mathrm{C}$ in LB-Amp. These were then added to $90 \mathrm{ml} \mathrm{LB}-A \mathrm{mp}$ pre-warmed to $37^{\circ} \mathrm{C}$ and further incubated at $37^{\circ} \mathrm{C}$. The starting optical density at $600 \mathrm{~nm}$ was measured and tracked to reach a value of 0.6 at which point protein expression was induced with $0.1 \mu \mathrm{M}$ IPTG for $4-5$ hours at $25^{\circ} \mathrm{C} .25 \mathrm{ml}$ culture aliquots were used to harvest bacterial pellets at $2500 \mathrm{~g}, 15 \mathrm{~min}, \mathrm{RT}$. The pellets were frozen at $-80^{\circ} \mathrm{C}$ for up to 6 months. The frozen pellet was thawed on ice, resuspended in $10 \mathrm{ml} \mathrm{PBS}$ and lysed by sonication thrice at $30 \mathrm{sec}, 40 \%$ power, $50 \%$ cycle followed by treatment with $0.1 \%$ TritionX-100 for $20 \mathrm{~min}$ on ice. The cell debris was pelleted at $1500 \mathrm{~g}, 35 \mathrm{~min}, 4^{\circ} \mathrm{C}$ and the supernatant incubated with a $50 \%$ slurry of $200 \mu \mathrm{l}$ glutathione sepharose beads on a rotator at $4^{\circ} \mathrm{C}$ for 2 hours. The beads were then washed thrice at $500 \mathrm{~g}, 4 \mathrm{~min}, 4^{\circ} \mathrm{C}$ with $1 \mathrm{X}$ PBS, leaving about $100 \mu \mathrm{l} 1 \mathrm{X}$ PBS over the 
beads and stored at $4^{\circ} \mathrm{C}$ for up to 10 days. To check for amount of purified GST fusion protein, different volumes were boiled with laemlli buffer at $95^{\circ} \mathrm{C}$ for $5 \mathrm{~min}$, loaded onto a $10 \%$ mini-gel along with titrated amounts of BSA. The gel was stained with coomassie solution for $30 \mathrm{~min}$ and destained in water $\mathrm{O} / \mathrm{N}$. The GST fusion protein concentration was calculated based on comparison of band intensity with the BSA standard.

\subsubsection{Affinity purification using GST fusion proteins}

B cells were grown to about $70 \%$ confluency and harvested at $300 \mathrm{~g}$, RT for $5 \mathrm{~min}$. The cell pellet was resuspended in 1X PBS and the cells were counted. The cells were harvested, resuspended in $10 \mathrm{ml} \mathrm{R0}$ and starved for $30 \mathrm{~min}$ at $37^{\circ} \mathrm{C}$ with regular mixing. 30 million cells per sample were resuspended in $500 \mu \mathrm{l} 0$ and stimulated with anti-BCR antibodies for the stipulated time points or left unstimulated. The cells were harvested in the minicentrifuge, washed once with 1X PBS and then lysed with $600 \mu$ lysis buffer on ice for $10 \mathrm{~min}$. The cell debris was pelleted at $20000 \mathrm{~g}, 10 \mathrm{~min}, 4^{\circ} \mathrm{C}$ and the cell lysates were incubated with $15 \mu \mathrm{g}$ GST-fusion protein and $30 \mu$ glutathione sepharose beads for 2 hours at $4^{\circ} \mathrm{C}$ with constant rotation. The beads were then washed thrice with lysis buffer at $500 \mathrm{~g}, 4 \mathrm{~min}, 4^{\circ} \mathrm{C}$. The beads were mixed with $30 \mu \mathrm{l} 2 \mathrm{X}$ laemlli and boiled at $95^{\circ} \mathrm{C}$ for 5 min. The samples were analyzed by SDS-PAGE and immunoblotting.

\subsubsection{2 $\mathrm{Ca}^{2+}$ measurements}

For monitoring $\mathrm{Ca}^{2+}$ flux following BCR stimulation, the $\mathrm{Ca}^{2+}$ indicator Indo1-AM, which is an acetoxymethyl ester of the fluorescent dye Indo1 was used. The dye enters the cells when uncharged, gets cleaved by intracellular esterase's to become charged and hence is trapped in the cells. Binding of $\mathrm{Ca}^{2+}$ to the dye leads to a shift in its emission spectra from $530 \mathrm{~nm}$ to $405 \mathrm{~nm}$ and hence gives a measure of changes in intracellular $\mathrm{Ca}^{2+}$ levels. The cells were grown to a confluency of about $70 \%$ to minimize pre-activation. 1 million cells per measurement were harvested at $300 \mathrm{~g}$, RT for $5 \mathrm{~min}$ and each set of samples was resuspended in $500 \mu \mathrm{R} 10$. The cells were transferred to a light protected eppendorf and a staining mix consisting of $200 \mu \mathrm{R} 10,0.7 \mu \mathrm{l}$ Indo-1 AM ( $1 \mathrm{mM}$ stock in DMSO) and $2 \mu \mathrm{l}$ Pluronic acid F-217 (5\% stock) was added to the tube. The tube was vortexed for $5 \mathrm{sec}$ and kept on the thermomixer for $30 \mathrm{~min}$ at $30^{\circ} \mathrm{C}$ and $600 \mathrm{rpm}$. The cells were then washed twice in Krebs Ringer solution (pre-warmed to RT) at $300 \mathrm{~g}$, RT for 2 min and finally resuspended in $300 \mu \mathrm{l}$ Krebs Ringer solution per million cells. The cells were incubated at $30^{\circ} \mathrm{C}$ at $300 \mathrm{rpm}$ for $30 \mathrm{~min}$ on the thermo mixer for revival. For the measurement, a basal $\mathrm{Ca}^{2+}$ level was measured for $30 \mathrm{sec}$ after which the sample was stimulated with the anti-BCR antibodies. Measurements were done at the LSRII, data acquisition using FACSDIVA and data analysis using FlowJo and Microsoft Excel.

\subsubsection{Mass spectrometry}


The human mlgE-ITT interactome was elucidated by purifying interaction partners form SILAC labeled B cells. All reagents and flasks used for the experiment were autoclaved or sterile packaged to prevent and dust contamination. Wild-type DG75 B cells were grown in "Heavy" or "Light" SILAC medium for 1 week for incorporation of the amino acid labels. The "Heavy" label contained L-Arginine $\left({ }^{13} \mathrm{C}_{6}\right)$ and L-Lysine $\left(\mathrm{D}_{4}\right)$ whereas the light label had these amino acids in their normal molecular weight. The cells were expanded to about $70 \%$ confluency and harvested at $300 \mathrm{~g}$, RT for $4 \mathrm{~min}$. The cell pellet was resuspended in 1X PBS and the cells were counted. The cells were harvested and 150 million cells per sample were lysed with $3 \mathrm{ml}$ lysis buffer (1\% NP40) on ice for $15 \mathrm{~min}$. The cell debris was pelleted at $20000 \mathrm{~g}, 10 \mathrm{~min}, 4^{\circ} \mathrm{C}$ and the cell lysates were incubated with 1 $\mu \mathrm{M}$ of the biotinylated peptide. The "Heavy" labeled cells were treated with $1 \mu \mathrm{M}$ human- $\varepsilon^{-}$ phospho-ITT peptide whereas the "Light" labeled cells were treated with $1 \mu \mathrm{M}$ nonphosphorylated human- $\varepsilon$-ITT peptide. $200 \mu \mathrm{l}$ streptavidin beads were added and the samples were incubated for 3 hours at $4^{\circ} \mathrm{C}$ with constant rotation. The beads were then washed thrice with lysis buffer at $500 \mathrm{~g}, 4 \mathrm{~min}, 4^{\circ} \mathrm{C}$. In the end, both samples were combined and mixed with $120 \mu \mathrm{l}$ 1X LDS boiling buffer (prepared from stock solutions of 10X NuPAGE sample reducing buffer and 4X NuPAGE LDS sample buffer in $\mathrm{dd}_{2} \mathrm{O}$ ) and boiled at $95^{\circ} \mathrm{C}$ for exactly $5 \mathrm{~min}$. The beads were spun down in the minicentrifuge at $2000 \mathrm{~g}$ for $15 \mathrm{sec}$ and the supernatant was carefully removed and sent for mass spectrometry analysis to the Proteomics Core Facility at the University Medical Center Goettingen. There, the sample was size separated using SDS-PAGE and 23 gel bands encompassing the entire interactome were treated and analyzed by tandem mass spectrometry-MS/MS. The data was initially processed by the core facility and later by me using the Perseus software. In Perseus, the $\log _{2}$ transformed values of Heavy/Light $(\mathrm{H} / \mathrm{L})$ normalized ratio and $\log _{10}$ transformed values of intensity were created. The data was then filtered using the Significance B function and the scatter plot was generated. The proteins that had a $\mathrm{H} / \mathrm{L}$ normalized ratio of more than 2.0 were considered to be statistically relevant interaction partners of the human- $\varepsilon$-phospho-ITT peptide. The scatter plot and list of enriched proteins were exported to CorelDraw for final processing. 


\section{References}

- Achatz, G., Nitschke, L., and Lamers, M.C. (1997). Effect of transmembrane and cytoplasmic domains of IgE on the IgE response. Science 276, 409-411.

- Achatz, G., Achatz-Straussberger, G., Feichtner, S., Koenigsberger, S., Lenz, S., Peckl-Schmid, D., Zaborsky, N., and Lamers, M. (2010). The Biology of IgE: Molecular Mechanism Restraining Potentially Dangerous High Serum IgE Titres In Vivo. In Cancer and IgE: Introducing the Concept of AllergoOncology, M.L. Penichet, and E. Jensen-Jarolim, eds. (Totowa, NJ: Humana Press), pp. 13-36.

- Ackermann, J.A., Radtke, D., Maurberger, A., Winkler, T.H., and Nitschke, L. (2011). Grb2 regulates B-cell maturation, B-cell memory responses and inhibits B-cell $\mathrm{Ca}+$ signalling. EMBO J. 30, 1621-1633.

- Allen, J.E., and Maizels, R.M. (2011). Diversity and dialogue in immunity to helminths. Nat. Rev. Immunol. 11, 375-388.

- Baba, Y., and Kurosaki, T. (2011). Impact of Ca2+ signaling on B cell function. Trends Immunol. 32, 589-594.

- Baba, Y., Matsumoto, M., and Kurosaki, T. (2014). Calcium signaling in B cells: Regulation of cytosolic Ca2+ increase and its sensor molecules, STIM1 and STIM2. Mol. Immunol. 62, 339-343.

- Batista, F.D., and Harwood, N.E. (2009). The who, how and where of antigen presentation to B cells. Nat. Rev. Immunol. 9, 15-27.

- Batista, F.D., Efremov, D.G., Tkach, T., and Burrone, O.R. (1995). Characterization and expression of alternatively spliced $\lg \mathrm{E}$ heavy chain trabscripts produced by peripheral blood lymphocytes. Nucleic Acids Res. 154, 209-218.

- Batista, F.D., Anand, S., Presani, G., Efremov, D.G., and Burrone, O.R. (1996). The Two Membrane Isoforms of Human IgE Assemble into Functionally Distinct B Cell Antigen Receptors. J. Exp. Med. 184, 2197 LP-2206.

- Von Behring, E., and Shibasaburo, K. (1890). Ueber das zutandekommen der diphtherie-immunitat und der tetanus-immunitat bei thieren. Dtsch. Medizinsche Wochenschrift 1113-1114.

- Ben-Bassat, H., Goldblum, N., Mitrani, S., Goldblum, T., Yoffey, J.M., Cohen, M.M., Bentwich, Z., Ramot, B., Klein, E., and Klein, G. (1977). Establishment in continuous culture of a new type of lymphocyte from a "Burkitt like" malignant lymphoma (line D.G.-75).

- Berkowska, M.A., Heeringa, J.J., Hajdarbegovic, E., Van Der Burg, M., Thio, H.B., Van Hagen, P.M., Boon, L., Orfao, A., Van Dongen, J.J.M., and Van Zelm, M.C. (2014). Human IgE+ B cells are derived from T cell-dependent and T cell-independent pathways. J. Allergy Clin. Immunol. 134, 688-697.e6.

- Bhattacharya, D., Cheah, M.T., Franco, C.B., Hosen, N., Pin, C.L., Sha, W.C., and Weissman, I.L. (2007). Transcriptional profiling of antigen-dependent murine B cell differentiation and memory formation. J Immunol 179, 6808-6819. 
- Bhattacharyya, S., Deb, J., Patra, A.K., Thuy Pham, D.A., Chen, W., Vaeth, M., Berberich-Siebelt, F., Klein-Hessling, S., Lamperti, E.D., Reifenberg, K., et al. (2011). NFATc1 affects mouse splenic B cell function by controlling the calcineurin--NFAT signaling network. J. Exp. Med. 208, 823-839.

- Brack, C., Hirama, M., Lenhard-Schuller, R., and Tonegawa, S. (1978). A complete immunoglobulin gene is created by somatic recombination. Cell 15, 1-14.

- Brooks, S.R., Kirkham, P.M., Freeberg, L., and Carter, R.H. (2004). Binding of cytoplasmic proteins to the CD19 intracellular domain is high affinity, competitive, and multimeric. J. Immunol. 172, 7556-7564.

- Buday, L., Egan, S.E., Viciana, P.R., Cantrell, D.A., and Downward, J. (1994). A complex of Grb2 adaptor protein, Sos exchange factor, and a 36-kDa membranebound tyrosine phosphoprotein is implicated in Ras activation in T cells. J. Biol. Chem. 269, 9019-9023.

- Buhl, A.M., and Cambier, J.C. (1999). Phosphorylation of CD19 Y484 and Y515, and Linked Activation of Phosphatidylinositol 3-Kinase, Are Required for B Cell Antigen Receptor-Mediated Activation of Bruton's Tyrosine Kinase. J. Immunol. 162, 44384446.

- Butt, D., Chan, T.D., Bourne, K., Hermes, J.R., Nguyen, A., Statham, A., O'Reilly, L.A., Strasser, A., Price, S., Schofield, P., et al. (2015). FAS Inactivation Releases Unconventional Germinal Center B Cells that Escape Antigen Control and Drive IgE and Autoantibody Production. Immunity 42, 890-902.

- Campbell, K.S., Bedzyk, W.D., and Cambier, J.C. (1995). Manipulation of B cell antigen receptor tyrosine phosphorylation using aluminum fluoride and sodium orthovanadate. Mol. Immunol. 32, 1283-1294.

- Chen, H.Y., Liu, F.-T., Hou, C.M.H., Huang, J.S.W., Sharma, B.B., and Chang, T.W. (2002). Monoclonal antibodies against the $\mathrm{C}($ epsilon) $\mathrm{mX}$ domain of human membrane-bound $\lg \mathrm{E}$ and their potential use for targeting IgE-expressing B cells. Int. Arch. Allergy Immunol. 128, 315-324.

- Chen, X., Pan, W., Sui, Y., Li, H., Shi, X., Guo, X., Qi, H., Xu, C., and Liu, W. (2015). Acidic phospholipids govern the enhanced activation of lgG-B cell receptor. Nat. Commun. 6, 8552.

- Cooper, M.D. (2015). The early history of B cells. Nat. Rev. Immunol. 15, 191-197.

- Coughlin, J.J., Stang, S.L., Dower, N. a, and Stone, J.C. (2005). RasGRP1 and RasGRP3 regulate $B$ cell proliferation by facilitating $B$ cell receptor-Ras signaling. J. Immunol. 175, 7179-7184.

- Cox, J., and Mann, M. (2008). MaxQuant enables high peptide identification rates, individualized p.p.b.-range mass accuracies and proteome-wide protein quantification. Nat. Biotechnol. 26, 1367-1372.

- Dixon, F.J., Maurer, P.H., and Deichmiller, M.P. (1954). Primary and Specific Anamnestic Antibody Responses of Rabbits to Heterologous Serum Protein Antigens. J. Immunol. 72, 179-186.

- Dogan, I., Bertocci, B., Vilmont, V., Delbos, F., Mégret, J., Storck, S., Reynaud, C.-A., 
and Weill, J.-C. (2009). Multiple layers of B cell memory with different effector functions. Nat. Immunol. 10, 1292-1299.

- Engelke, M., Engels, N., Dittmann, K., Stork, B., and Wienands, J. (2007). Ca2+ signaling in antigen receptor-activated B lymphocytes. Immunol. Rev. 218, 235-246.

- Engels, N., and Wienands, J. (2011). The signaling tool box for tyrosine-based costimulation of lymphocytes. Curr. Opin. Immunol. 23, 324-329.

- Engels, N., Wollscheid, B., and Wienands, J. (2001). Association of SLP-65/BLNK with the B cell antigen receptor through a non-ITAM tyrosine of Ig-?? Eur. J. Immunol. 31, 2126-2134.

- Engels, N., König, L.M., Heemann, C., Lutz, J., Tsubata, T., Griep, S., Schrader, V., and Wienands, J. (2009). Recruitment of the cytoplasmic adaptor Grb2 to surface IgG and $\operatorname{lgE}$ provides antigen receptor-intrinsic costimulation to class-switched B cells. Nat. Immunol. 10, 1018-1025.

- Engels, N., König, L.M., Schulze, W., Radtke, D., Vanshylla, K., Lutz, J., Winkler, T.H., Nitschke, L., and Wienands, J. (2014). The immunoglobulin tail tyrosine motif upgrades memory-type BCRs by incorporating a Grb2-Btk signalling module. Nat. Commun. 5:5456, 1-11.

- Feske, S. (2007). Calcium signalling in lymphocyte activation and disease. Nat. Rev. Immunol. 7, 690-702.

- Flajnik, M.F. (2002). Comparative analyses of immunoglobulin genes: surprises and portents. Nat. Rev. Immunol. 2, 688-698.

- Fujimoto, M., Poe, J.C., Hasegawa, M., and Tedder, T.F. (2000). CD19 regulates intrinsic $B$ lymphocyte signal transduction and activation through a novel mechanism of processive amplification. Immunol. Res. 22, 281-298.

- Fujimoto, M., Poe, J.C., Hasegawa, M., and Tedder, T.F. (2001). CD19 Amplification of B Lymphocyte Ca2+ Responses: A role for Lyn sequestration in extinguishing negative regulation. J. Biol. Chem. 276, 44820-44827.

- Gauvreau, G.M., Harris, J.M., Boulet, L.-P., Scheerens, H., Fitzgerald, J.M., Putnam, W.S., Cockcroft, D.W., Davis, B.E., Leigh, R., Zheng, Y., et al. (2014). Targeting membrane-expressed IgE B cell receptor with an antibody to the M1 prime epitope reduces IgE production. Sci. Transl. Med. 6, 243ra85.

- Good, K.L., and Tangye, S.G. (2007). Decreased expression of Kruppel-like factors in memory $B$ cells induces the rapid response typical of secondary antibody responses. Proc. Natl. Acad. Sci. U. S. A. 104, 13420-13425.

- Goodnow, C.C., Vinuesa, C.G., Randall, K.L., Mackay, F., and Brink, R. (2010). Control systems and decision making for antibody production. Nat. Immunol. 11, 681688.

- Gould, H.J., and Sutton, B.J. (2008). IgE in allergy and asthma today. Nat. Rev. Immunol. 8, 205-217.

- Gray, D. (1993). Immunological memory. Annu Rev Immunol 11, 49-77.

- Gurish, M.F., Bryce, P.J., Tao, H., Kisselgof, A.B., Thornton, E.M., Miller, H.R., Friend, D.S., and Oettgen, H.C. (2004). IgE Enhances Parasite Clearance and Regulates 
Mast Cell Responses in Mice Infected with Trichinella spiralis. J. Immunol. 172, 1139_ 1145.

- Hallstrand, T.S., Sprenger, J.D., Agosti, J.M., Longton, G.M., Witherspoon, R.P., and Henderson, W.R. (2004). Long-term acquisition of allergen-specific IgE and asthma following allogeneic bone marrow transplantation from allergic donors. Blood 104, 3086-3090.

- Hangartner, L., Zinkernagel, R.M., and Hengartner, H. (2006). Antiviral antibody responses: the two extremes of a wide spectrum. Nat. Rev. Immunol. 6, 231-243.

- Haniuda, K., Fukao, S., Kodama, T., Hasegawa, H., and Kitamura, D. (2016). Autonomous membrane IgE signaling prevents IgE-memory formation. Nat. Immunol. 17, 1109-1117.

- Hao, Z., Duncan, G.S., Seagal, J., Su, Y.W., Hong, C., Haight, J., Chen, N.J., Elia, A., Wakeham, A., Li, W.Y., et al. (2008). Fas Receptor Expression in Germinal-Center B Cells Is Essential for T and B Lymphocyte Homeostasis. Immunity 29, 615-627.

- Havran, W.L., DiGiusto, D.L., and Cambier, J.C. (1984). mlgM:mlgD ratios on B cells: mean mlgD expression exceeds mlgM by 10 -fold on most splenic B cells. J. Immunol. 132, 1712-1716.

- Hawkins, E.D., Oliaro, J., Kallies, A., Belz, G.T., Filby, A., Hogan, T., Haynes, N., Ramsbottom, K.M., Van Ham, V., Kinwell, T., et al. (2013). Regulation of asymmetric cell division and polarity by Scribble is not required for humoral immunity. Nat. Commun. 4, 1801.

- He, J.-S., Meyer-Hermann, M., Xiangying, D., Zuan, L.Y., Jones, L.A., Ramakrishna, L., de Vries, V.C., Dolpady, J., Aina, H., Joseph, S., et al. (2013). The distinctive germinal center phase of $\mathrm{IgE}+\mathrm{B}$ lymphocytes limits their contribution to the classical memory response. J. Exp. Med. 210, 2755-2771.

- Hebeis, B.J., Klenovsek, K., Rohwer, P., Ritter, U., Schneider, A., Mach, M., and Winkler, T.H. (2004). Activation of Virus-specific Memory B Cells in the Absence of $T$ Cell Help. J. Exp. Med. 199, 593-602.

- Herzog, S., Reth, M., and Jumaa, H. (2009). Regulation of B-cell proliferation and differentiation by pre-B-cell receptor signalling. Nat. Rev. Immunol. 9, 195-205.

- Holgate, S.T., and Polosa, R. (2008). Treatment strategies for allergy and asthma. Nat Rev Immunol 8, 218-230.

- Hombach, J., Tsubata, T., Leclercq, L., Stappert, H., and Reth, M. (1990). Molecular components of the B-cell antigen receptor complex of the IgM class. Nature 343, 760762.

- Honjo, T., Kinoshita, K., and Muramatsu, M. (2002). Molecular Mechanism of Classswitch Recombination: Linkage with Somatic Hypermutation. Annu. Rev. Immunol. 20, 165-196.

- Iwasaki, A., and Medzhitov, R. (2015). Control of adaptive immunity by the innate immune system. Nat. Immunol. 16, 343-353.

- Janeway, C.A., and Medzhitov, R. (2002). Innate Immune Recognition. Annu. Rev. Immunol. 20, 197-216. 
- Jang, I.K., Zhang, J., Chiang, Y.J., Kole, H.K., Cronshaw, D.G., Zou, Y., and Gu, H. (2010). Grb2 functions at the top of the T-cell antigen receptor-induced tyrosine kinase cascade to control thymic selection. Proc. Natl. Acad. Sci. 107, 10620-10625.

- Jang, I.K., Cronshaw, D.G., Xie, L., Fang, G., Zhang, J., Oh, H., Fu, Y.-X., Gu, H., and Zou, Y. (2011). Growth-factor receptor-bound protein-2 (Grb2) signaling in B cells controls lymphoid follicle organization and germinal center reaction. Proc. Natl. Acad. Sci. U. S. A. 108, 7926-7931.

- Johnson, S. a, Pleiman, C.M., Pao, L., Schneringer, J., Hippen, K., and Cambier, J.C. (1995). Phosphorylated immunoreceptor signaling motifs (ITAMs) exhibit unique abilities to bind and activate Lyn and Syk tyrosine kinases. J. Immunol. 155, 45964603.

- Jou, S., Carpino, N., Takahashi, Y., Chao, J., Carpino, N., Wang, D., Ihle, J.N., and Piekorz, R. (2002). Essential, Nonredundant Role for the Phosphoinositide 3-Kinase p110 $\delta$ in Signaling by the B-Cell Receptor Complex Essential , Nonredundant Role for the Phosphoinositide 3-Kinase p110 $\square$ in Signaling by the B-Cell Receptor Complex. Mol. Cell. Biol. 22, 8580-8591.

- Kaisho, T. (1997). The Roles of 1 Heavy Chain Membrane Expression and Cytoplasmic Tail in IgG1 Responses. Science (80-. ). 276, 412-415.

- Kawabe, T., Naka, T., Yoshida, K., Tanaka, T., Fujiwara, H., Suematsu, S., Yoshida, N., Kishimoto, T., and Kikutani, H. (1994). The immune responses in CD40-deficient mice: Impaired immunoglobulin class switching and germinal center formation. Immunity 1, 167-178.

- Kearney, D.G.B.L.M.H.J.F. (1986). Posttranslational Association of Immunoglobulin Heavy Chain. J. Cell Biol. 102, 1558-1566.

- Keshvara, L.M., Isaacson, C.C., Yankee, T.M., Sarac, R., Harrison, M.L., and Geahlen, R.L. (1998). Syk- and Lyn-dependent phosphorylation of Syk on multiple tyrosines following $B$ cell activation includes a site that negatively regulates signaling. J. Immunol. 161, 5276-5283.

- Kim, K.M., Alber, G., Weiser, P., and Reth, M. (1993). Differential signaling through the Ig-alpha and Ig-beta components of the B cell antigen receptor. Eur. J. Immunol. 23, 911-916.

- Kim, Y.M., Pan, J.Y., Korbel, G.A., Peperzak, V., Boes, M., and Ploegh, H.L. (2006). Monovalent ligation of the $B$ cell receptor induces receptor activation but fails to promote antigen presentation. Proc Natl Acad Sci U S A 103, 3327-3332.

- Kläsener, K., Maity, P.C., Hobeika, E., Yang, J., and Reth, M. (2014). B cell activation involves nanoscale receptor reorganizations and inside-out signaling by Syk. Elife 3, e02069.

- Klein, G., Giovanella, B., Westman, A., Stehlin, J.S., and Mumford, D. (1975). An EBV-genome-negative cell line established from an American Burkitt lymphoma; receptor characteristics. EBV infectibility and permanent conversion into EBV-positive sublines by in vitro infection. Intervirology 5, 319-334.

- Klein, U., Tu, Y., Stolovitzky, G.A., Keller, J.L., Haddad Jr., J., Miljkovic, V., Cattoretti, 
G., Califano, A., and Dalla-Favera, R. (2003). Transcriptional analysis of the B cell germinal center reaction. Proc Natl Acad Sci U S A 100, 2639-2644.

- Knittler, M.R., Dirks, S., and Haas, I.G. (1995). Molecular chaperones involved in protein degradation in the endoplasmic reticulum: quantitative interaction of the heat shock cognate protein $\mathrm{BiP}$ with partially folded immunoglobulin light chains that are degraded in the endoplasmic reticulum. Proc. Natl. Acad. Sci. U. S. A. 92, 1764-1768.

- Kometani, K., Nakagawa, R., Shinnakasu, R., Kaji, T., Rybouchkin, A., Moriyama, S., Furukawa, K., Koseki, H., Takemori, T., and Kurosaki, T. (2013). Repression of the Transcription Factor Bach2 Contributes to Predisposition of IgG1 Memory B Cells toward Plasma Cell Differentiation. Immunity 39, 136-147.

- Kumar, G., Wang, S., Gupta, S., and Nel, A. (1995). The membrane immunoglobulin receptor utilizes a Shc/Grb2/hSOS complex for activation of the mitogen-activated protein kinase cascade in a B-cell line. Biochem. J. 307 ( Pt 1, 215-223.

- Kurosaki, T., and Tsukada, S. (2000). BLNK: connecting Syk and Btk to calcium signals. Immunity 12, 1-5.

- Kurosaki, T., Johnson, S. a, Pao, L., Sada, K., Yamamura, H., and Cambier, J.C. (1995). Role of the Syk autophosphorylation site and $\mathrm{SH} 2$ domains in B cell antigen receptor signaling. J. Exp. Med. 182, 1815-1823.

- Li, N., Batzer, A., Daly, R., Yajnik, V., Skolnik, E., Chardin, P., Bar-Sagi, D., Margolis, B., and Schlessinger, J. (1993). Guanine-nucleotide-releasing factor hSos1 binds to Grb2 and links receptor tyrosine kinases to Ras signalling. Nature 363, 85-88.

- Liu, W., Meckel, T., Tolar, P., Sohn, H.W., and Pierce, S.K. (2010). Intrinsic properties of immunoglobulin IgG1 isotype-switched B cell receptors promote microclustering and the initiation of signaling. Immunity $32,778-789$.

- Liu, W., Chen, E., Zhao, X.W., Wan, Z.P., Gao, Y.R., Davey, A., Huang, E., Zhang, L., Crocetti, J., Sandoval, G., et al. (2012). The Scaffolding Protein Synapse-Associated Protein 97 Is Required for Enhanced Signaling Through Isotype-Switched IgG Memory B Cell Receptors. Sci. Signal. 5, ra54-ra54.

- Lutz, J., Dittmann, K., Bösl, M.R., Winkler, T.H., Wienands, J., and Engels, N. (2015). Reactivation of IgG-switched memory B cells by BCR-intrinsic signal amplification promotes IgG antibody production. Nat. Commun. 6, 8575.

- MacKenzie, T., and Dosch, H.M. (1989). Clonal and molecular characeristics of the human IgE-committed B cell subset. J Exp Med 169.

- Martin, S.W., and Goodnow, C.C. (2002). Burst-enhancing role of the IgG membrane tail as a molecular determinant of memory. Nat. Immunol. 3, 182-188.

- Matsuuchi, L., Gold, M.R., Travis, a, Grosschedl, R., DeFranco, a L., and Kelly, R.B. (1992). The membrane IgM-associated proteins MB-1 and Ig-beta are sufficient to promote surface expression of a partially functional B-cell antigen receptor in a nonlymphoid cell line. Proc. Natl. Acad. Sci. U. S. A. 89, 3404-3408.

- Maude, S.L., Frey, N., Shaw, P. a., Aplenc, R., Barrett, D.M., Bunin, N.J., Chew, A., Gonzalez, V.E., Zheng, Z., Lacey, S.F., et al. (2014). Chimeric Antigen Receptor T Cells for Sustained Remissions in Leukemia. N. Engl. J. Med. 371, 1507-1517. 
- McHeyzer-Williams, M., Okitsu, S., Wang, N., and McHeyzer-Williams, L. (2012). Molecular programming of B cell memory. Nat. Rev. Immunol. 12, 24-34.

- McHeyzer-Williams, M.G., Nossal, G.J., and Lalor, P.A. (1991). Molecular characterization of single memory B cells. Nature 350, 502-505.

- Melchers, F., Karasuyama, H., Haasner, D., Bauer, S., Kudo, A., Sakaguchi, N., Jameson, B., and Rolink, A. (1993). The surrogate light chain in B-cell development. Immunol. Today 14, 60-68.

- Metzger, H. (1992). American Association of Immunologists Transmembrane Signaling: the Joy of Aggregation. J. Immunol. 149, 1477-1487.

- Mitre, E., and Nutman, T.B. (2006). IgE memory: Persistence of antigen-specific IgE responses years after treatment of human filarial infections. J. Allergy Clin. Immunol. 117, 939-945.

- Morita, S., Kojima, T., and Kitamura, T. (2000). Plat-E: an efficient and stable system for transient packaging of retroviruses. Gene Ther. 7, 1063-1066.

- Muller, M.R., and Rao, A. (2010). NFAT, immunity and cancer: a transcription factor comes of age. Nat Rev Immunol 10, 645-656.

- Mußmann, R., Wilson, M., Marcur, A., Courtet, M., and Du Pasquier, L. (1996). Membrane exon sequences of the three Xenopus Ig classes explain the evolutionary origin of mammalian isotypes. Eur. J. Immunol. 26, 409-414.

- Neumann, K., Oellerich, T., Urlaub, H., and Wienands, J. (2009). The B-lymphoid Grb2 interaction code. Immunol. Rev. 232, 135-149.

- Okkenhaug, K., and Vanhaesebroeck, B. (2003). PI3K in lymphocyte development, differentiation and activation. Nat. Rev. - Immunol. 3, 317-330.

- Pape, K.A., Taylor, J.J., Maul, R.W., Gearhart, P.J., and Jenkins, M.K. (2011). Different B Cell Populations Mediate Early and Late Memory During an Endogenous Immune Response. Science (80-. ). 331, 1203 LP-1207.

- Paul, E. (1900). On immunity with special reference to cell life. In Proceedings of the Royal Society of London, pp. 424-448.

- Poe, J.C., Fujimoto, M., Jansen, P.J., Miller, A.S., and Tedder, T.F. (2000). CD22 forms a quaternary complex with SHIP, Grb2, and Shc: A pathway for regulation of B lymphocyte antigen receptor-induced calcium flux. J. Biol. Chem. 275, 17420-17427.

- Pogue, S.L., Kurosaki, T., Bolen, J., and Herbst, R. (2000). B cell antigen receptorinduced activation of Akt promotes B cell survival and is dependent on Syk kinase. J. Immunol. 165, 1300-1306.

- Radbruch, A., Muehlinghaus, G., Luger, E.O., Inamine, A., Smith, K.G.C., Dörner, T., and Hiepe, F. (2006). Competence and competition: the challenge of becoming a long-lived plasma cell. Nat. Rev. Immunol. 6, 741-750.

- Ramadani, F., Bowen, H., Upton, N., Hobson, P.S., Chan, Y.C., Chen, J.B., Chang, T.W., Mcdonnell, J.M., Sutton, B.J., Fear, D.J., et al. (2016). Ontogeny of human IgEexpressing B cells and plasma cells. Allergy Eur. J. Allergy Clin. Immunol.

- Reth, M. (1989). Antigen receptor tail clue. Nature 338, 383-384.

- Reth, M. (1992). Antigen receptors on B lymphocytes. Annu. Rev. Immunol. 10, 97- 
121.

- Reth, M., and Nielsen, P. (2014). Signaling circuits in early B-cell development (Elsevier Inc.).

- Rolli, V., Gallwitz, M., Wossning, T., Flemming, A., Schamel, W.W.A., Zürn, C., and Reth, M. (2002). Amplification of $B$ cell antigen receptor signaling by a Syk/ITAM positive feedback loop. Mol. Cell 10, 1057-1069.

- Roose, J.P., Mollenauer, M., Ho, M., Kurosaki, T., and Weiss, A. (2007). Unusual interplay of two types of Ras activators, RasGRP and SOS, establishes sensitive and robust Ras activation in lymphocytes. Mol Cell Biol 27, 2732-2745.

- Saito, K., Scharenberg, A.M., and Kinet, J.P. (2001). Interaction between the Btk PH Domain and Phosphatidylinositol-3,4,5-trisphosphate Directly Regulates Btk. J. Biol. Chem. 276, 16201-16206.

- Schamel, W.W., and Reth, M. (2000a). Stability of the B cell antigen receptor complex. Mol. Immunol. 37, 253-259.

- Schamel, W.W., and Reth, M. (2000b). Monomeric and oligomeric complexes of the B cell antigen receptor. Immunity 13, 5-14.

- Schulman, E.S. (2001). Development of a monoclonal anti-immunoglobulin E antibody (omalizumab) for the treatment of allergic respiratory disorders. Am. J. Respir. Crit. Care Med. 164, 6-11.

- Segal, A.W. (2005). How Neutrophils Kill Microbes. Annu. Rev. Immunol. 23, 197223.

- Selsted, M.E., and Ouellette, A.J. (2005). Mammalian defensins in the antimicrobial immune response. Nat. Immunol. 6, 551-557.

- De Silva, N.S., and Klein, U. (2015). Dynamics of B cells in germinal centres. Nat. Rev. Immunol. 15, 137-148.

- Simon, J.A., and Schreiber, S.L. (1995). Grb2 SH3 binding to peptides from Sos: evaluation of a general model for SH3-ligand interactions. Chem. Biol. 2, 53-60.

- Stork, B., Engelke, M., Frey, J., Horejsí, V., Hamm-Baarke, A., Schraven, B., Kurosaki, T., and Wienands, J. (2004). Grb2 and the non-T cell activation linker ntal constitute a Ca 2+-regulating signal circuit in B lymphocytes. Immunity 21, 681-691.

- Stork, B., Neumann, K., Goldbeck, I., Alers, S., Kähne, T., Naumann, M., Engelke, M., and Wienands, J. (2007). Subcellular localization of Grb2 by the adaptor protein Dok-3 restricts the intensity of Ca2+ signaling in B cells. EMBO J. 26, 1140-1149.

- Su, T.T., Guo, B., Kawakami, Y., Sommer, K., Chae, K., Humphries, L.A., Kato, R.M., Kang, S., Patrone, L., Wall, R., et al. (2002). PKC-beta controls I kappa B kinase lipid raft recruitment and activation in response to BCR signaling. Nat. Immunol. 3, 780786.

- Sutherland, C.L., Heath, A.W., Pelech, S.L., Young, P.R., and Gold, M.R. (1996). Differential activation of the ERK, JNK, and p38 mitogen-activated protein kinases by CD40 and the B cell antigen receptor. J. Immunol. 157, 3381-3390.

- Takeshi Tsubata, J.W.\& T.H. (1993). B-cell apoptosis induced by antigen receptor crosslinking is blocked by a T-cell signal through CD40. Nature 364, 645-648. 
- Talay, O., Yan, D., Brightbill, H.D., Straney, E.E.M., Zhou, M., Ladi, E., Lee, W.P., Egen, J.G., Austin, C.D., Xu, M., et al. (2012). IgE $\mathrm{E}^{+}$memory B cells and plasma cells generated through a germinal-center pathway. Nat. Immunol. 13, 396-404.

- Tamir, I., and Cambier, J.C. (1998). Antigen receptor signaling: integration of protein tyrosine kinase functions. Oncogene 17, 1353-1364.

- Tangye, S.G., Avery, D.T., Deenick, E.K., and Hodgkin, P.D. (2003). Intrinsic differences in the proliferation of naive and memory human $B$ cells as a mechanism for enhanced secondary immune responses. J. Immunol. 170, 686-694.

- Tolar, P., Sohn, H.W., Liu, W., and Pierce, S.K. (2009). The molecular assembly and organization of signaling active B-cell receptor oligomers. Immunol. Rev. 232, 34-41.

- Tomayko, M.M., Anderson, S.M., Brayton, C.E., Sadanand, S., Steinel, N.C., Behrens, T.W., and Shlomchik, M.J. (2008). Systematic Comparison of Gene Expression between Murine Memory and Naive B Cells Demonstrates That Memory B Cells Have Unique Signaling Capabilities. J. Immunol. 181, 27-38.

- Turner, H., and Kinet, J.P. (1999). Signalling through the high-affinity lgE receptor Fc epsilonRI. Nature 402, B24-B30.

- Venkitaraman, a R., Williams, G.T., Dariavach, P., and Neuberger, M.S. (1991). The B-cell antigen receptor of the five immunoglobulin classes. Nature 352, 777-781.

- Vivier, E. (2008). Functions of natural killer cells. Nat. Immunol. 9, 503-510.

- Volkmann, C., Brings, N., Becker, M., Hobeika, E., Yang, J., and Reth, M. (2016). Molecular requirements of the B-cell antigen receptor for sensing monovalent antigens. EMBO J. e201694177-n/a.

- Wan, Z., Zhang, S., Fan, Y., Liu, K., Du, F., Davey, A.M., Zhang, H., Han, W., Xiong, C., and Liu, W. (2013). B Cell Activation Is Regulated by the Stiffness Properties of the Substrate Presenting the Antigens. J. Immunol. 190, 4661-4675.

- Wan, Z., Chen, X., Chen, H., Ji, Q., Chen, Y., Wang, J., Cao, Y., Wang, F., Lou, J., Tang, Z., et al. (2015). The activation of IgM- or isotype-switched IgG- and IgE-BCR exhibits distinct mechanical force sensitivity and threshold. Elife 4, 1-24.

- Weisel, F.J., Zuccarino-Catania, G. V., Chikina, M., and Shlomchik, M.J. (2016). A Temporal Switch in the Germinal Center Determines Differential Output of Memory B and Plasma Cells. Immunity 44, 116-130.

- Werner, M., Hobeika, E., and Jumaa, H. (2010). Role of PI3K in the generation and survival of B cells. Immunol. Rev. 237, 55-71.

- Wesemann, D.R., Magee, J.M., Boboila, C., Calado, D.P., Gallagher, M.P., Portuguese, A.J., Manis, J.P., Zhou, X., Recher, M., Rajewsky, K., et al. (2011). Immature $B$ cells preferentially switch to $\lg E$ with increased direct $S \mu$ to $S \varepsilon$ recombination. J. Exp. Med. 208, 2733-2746.

- Wienands, J., and Engels, N. (2016). The Memory Function of the B Cell Antigen Receptor. In B Cell Receptor Signaling, T. Kurosaki, and J. Wienands, eds. (Cham: Springer International Publishing), pp. 107-121.

- Wienands, J., Larbolette, O., and Reth, M. (1996). Evidence for a preformed transducer complex organized by the B cell antigen receptor. Proc. Natl. Acad. Sci. U. 


\section{S. A. 93, 7865-7870.}

- Wienands, J., Schweikert, J., Wollscheid, B., Jumaa, H., Nielsen, P.J., and Reth, M. (1998). SLP-65: a new signaling component in B lymphocytes which requires expression of the antigen receptor for phosphorylation. J. Exp. Med. 188, 791-795.

- Winslow, M.M., Gallo, E.M., Neilson, J.R., and Crabtree, G.R. (2006). The calcineurin phosphatase complex modulates immunogenic B cell responses. Immunity 24, 141152.

- Wu, L.C., and Zarrin, A. a (2014). The production and regulation of $\lg E$ by the immune system. Nat. Rev. Immunol. 14, 247-259.

- Yang, J., and Reth, M. (2010). Oligomeric organization of the B-cell antigen receptor on resting cells. Nature 467, 465-469.

- Yang, Z., Sullivan, B.M., and Allen, C.D.C. (2012). Fluorescent In Vivo Detection Reveals that $\lg E+B$ Cells Are Restrained by an Intrinsic Cell Fate Predisposition. Immunity 36, 857-872.

- Yasuda, T., Sanjo, H., Pagès, G., Kawano, Y., Karasuyama, H., Pouysségur, J., Ogata, M., and Kurosaki, T. (2008). Erk Kinases Link Pre-B Cell Receptor Signaling to Transcriptional Events Required for Early B Cell Expansion. Immunity 28, 499-508.

- Yasuda, T., Hayakawa, F., Kurahashi, S., Sugimoto, K., Minami, Y., Tomita, A., and Naoe, T. (2012). B cell receptor-ERK1/2 signal cancels PAX5-dependent repression of BLIMP1 through PAX5 phosphorylation: a mechanism of antigen-triggering plasma cell differentiation. J. Immunol. 188, 6127-6134.

- Yefenof, E., Sanders, V.M., Uhr, J.W., and Vitetta, E.S. (1986). In vitro activation of murine antigen-specific memory B cells by a T-dependent antigen. J. Immunol. 137, 85-90.

- Yuan, D., Vitetta, E.S., and Kettman, J.R. (1977). Cell surface immunoglobulin. XX. Antibody responsiveness of subpopulations of $\mathrm{B}$ lymphocytes bearing different isotypes. J. Exp. Med. 145, 1421-1435.

- Zhang, K., Saxon, A., and Max, E.E. (1992). Two unusual forms of human immunoglobulin $E$ encoded by alternative RNA splicing of epsilon heavy chain membrane exons. J. Exp. Med. 176, 233-243.

- Zhang, Y., Wienands, J., Zürn, C., and Reth, M. (1998). Induction of the antigen receptor expression on $B$ lymphocytes results in rapid competence for signaling of SLP-65 and Syk. Eur. Mol. Biol. Organ. J. 17, 7304-7310.

- Zipfel, P.F., and Skerka, C. (2009). Complement regulators and inhibitory proteins. Nat. Rev. Immunol. 9, 729-740.

- Zuccarino-Catania, G. V, Sadanand, S., Weisel, F.J., Tomayko, M.M., Meng, H., Kleinstein, S.H., Good-Jacobson, K.L., and Shlomchik, M.J. (2014). CD80 and PD-L2 define functionally distinct memory $B$ cell subsets that are independent of antibody isotype. Nat. Immunol. 15, 631-637.

- Zúñiga-Pflücker, J.C. (2004). T-cell development made simple. Nat. Rev. Immunol. 4, 67-72. 


\section{Acknowledgments}

Niklas, it's a pleasure to work with you in your lab. Thank you for supervising me and being there to guide me at every step along the way. I learn so much from you every day and that's not just limited to B cells and science! Your faith in my work always helped me push through the harder times and kept me going. Thank you for everything. :)

I sincerely thank Prof. Dr. Jürgen Wienands for giving me the chance to work with you. Thank you for mentoring me and giving me opportunities to meet and discuss my ideas with the scientific community. Your support constantly encourages me to do better.

I would also like to thank Prof. Dr. Mathias Dobbelstein and Dr. Roland Dosch for guiding me throughout my $\mathrm{PhD}$ with ideas and suggestions as part of my thesis committee.

I thank Ines Heine for superb technical assistance and all other present/past members of the lab for the great times through the years. I am also grateful to Frau Teuteberg and Frau Schindler for help with the lengthy official paperwork and sometimes having to spend time translating it for me. Thanks to Sona, Pranali and Carina for the fun breaks from work. I am also thankful to the coffee machine that provided almost drinkable coffee.

Thank you Niklas, Anita, Christoffer and Arshiya for proof-reading my thesis which was probably not an easy task if one takes my typing skills into account.

I am also very grateful towards Steffen and Kerstin for the round-the-clock help and all the work you put in to make the Molecular Biology program so special.

Zhenya, thanks for being an amazing friend and reminding me of what was important at the times that I could not. I also thank Anita for the sports regimes and for being there as a friend. Thanks to Karthika, Priya and Shivani for the support and sometimes crazy advice and even though we are in different countries, you never let me feel the distance.

I thank my father for being my pillar, supporting my decisions and encouraging me to do my best. You inspire me to be a better person and help people. I cannot thank you enough for how much you do for me. Finally, I would also like to thank my family for the love and support you all give me. 


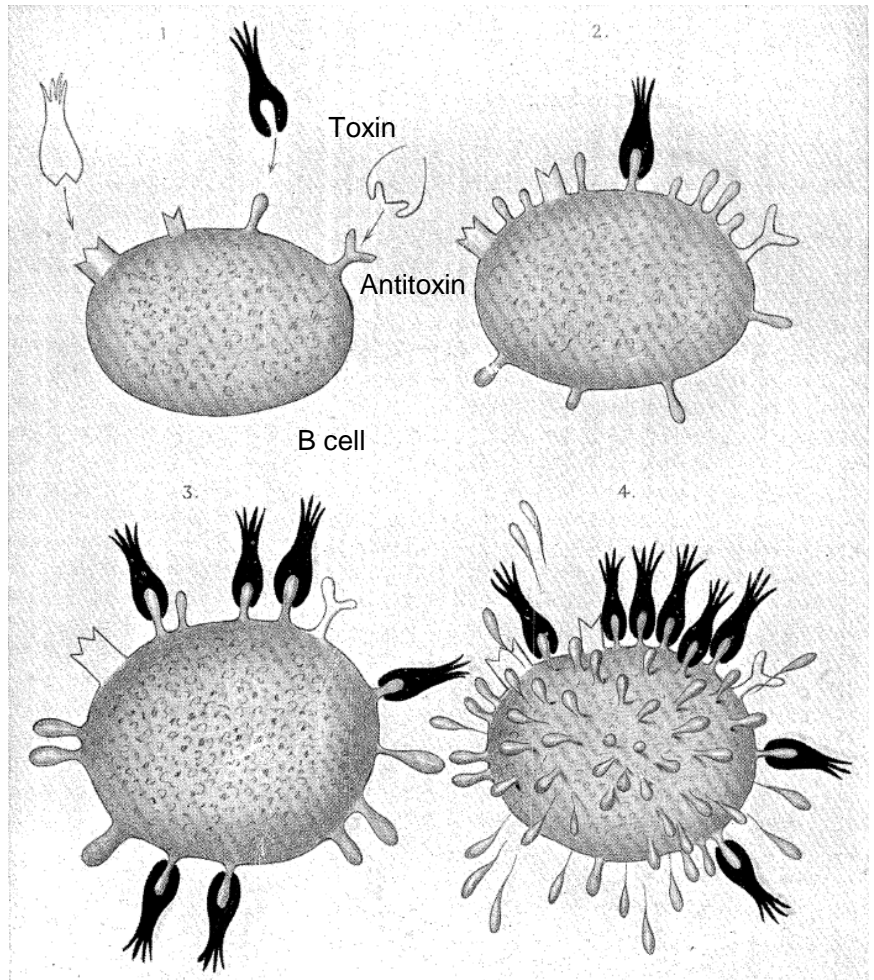

Figure modified from: Paul Ehrlich (1900). On immunity with special reference to cell life. In Proceedings of the Royal Society of London, pp. 424-448.

This was one of the first descriptions of the interaction between a toxin and an antitoxin (antibody). It was also the very first time that a mechanism involving a ligand and a ligandspecific receptor was proposed, a finding that also proved relevant for other fields of biology besides immunology. The so-called side-chain theory led to the awarding of the 1908 Nobel Prize in Physiology or Medicine to Paul Ehrlich. It is fascinating that Ehrlich not only correctly hypothesized the presence of a membrane-bound toxin-specific antitoxin, but also that binding of the toxin leads to activation of the cell and secretion of the very same antitoxin into the serum, providing long term protection. We now know this process as antigen-induced BCR signaling which can result in differentiation of B cells into antibody-secreting plasma cells.

It has been about 116 years since this model was first proposed. We have come a long way since then and our knowledge into antibody structure and function is now successfully applied in the clinics to specifically target molecules and cure diseases. However, despite the extensive progress in our understanding of antibodies, there are still new things to learn and discover about these molecules. This is something that inspires me to continue to move forward and make my own little contributions to the field of immunology. 


\section{List of abbreviations}

A

ADCC

$\mathrm{Ag}$

AP

BCR

Btk

C

CD

CRAC

Cyt

D

DAG

$\mathrm{ddH}_{2} \mathrm{O}$

$\mathrm{dKO}$

$\mathrm{E}$

EB

EMPD

ER

ERK

$\mathrm{F}$

$\mathrm{F}\left(a b^{\prime}\right)_{2}$

FACS

FceR

Fig.

G

GC

GEF

GFP

GRP

Grap

Grb2

GST

$\mathrm{H}$

$\mathrm{HC}$

$\mathrm{HL}$

HRP

I

$\lg$

$\lg \alpha$

$\lg \beta$
Alanine or Adenine

Antibody-dependent cell-mediated cytotoxicity

Antigen

Affinity purification

$B$ cell antigen receptor

Bruton tyrosine kinase

Cysteine or Cytosine

Cluster of differentiation

$\mathrm{Ca}^{2+}$ release-activated channel

cytoplasmic

Aspartic acid

Diacylglycerol

Double distilled water

Double knock out

Glutamic acid

EcoBlast (Ecotropic receptor and blasticidin resistance)

Extracellular membrane-proximal domain

Endoplasmic reticulum

Extracellular signal regulated kinase

Phenylalanine

Dimer of antigen binding fragment

Fluorescence activated cell sorting

Fc epsilon receptor

Figure

Glycine or Guanine

Germinal center

Guanine nucleotide exchange factor

Green fluorescent protein

Guanyl nucleotide-releasing protein

Grb2 related adaptor protein

Growth factor receptor-bound protein 2

Glutathione S-transferase

Histidine

Heavy chain

Immunoglobulin heavy chain and immunoglobulin light chain

Horse radish peroxidase

Isoleucine

Immunoglobulin

Immunoglobulin alpha

Immunoglobulin beta 


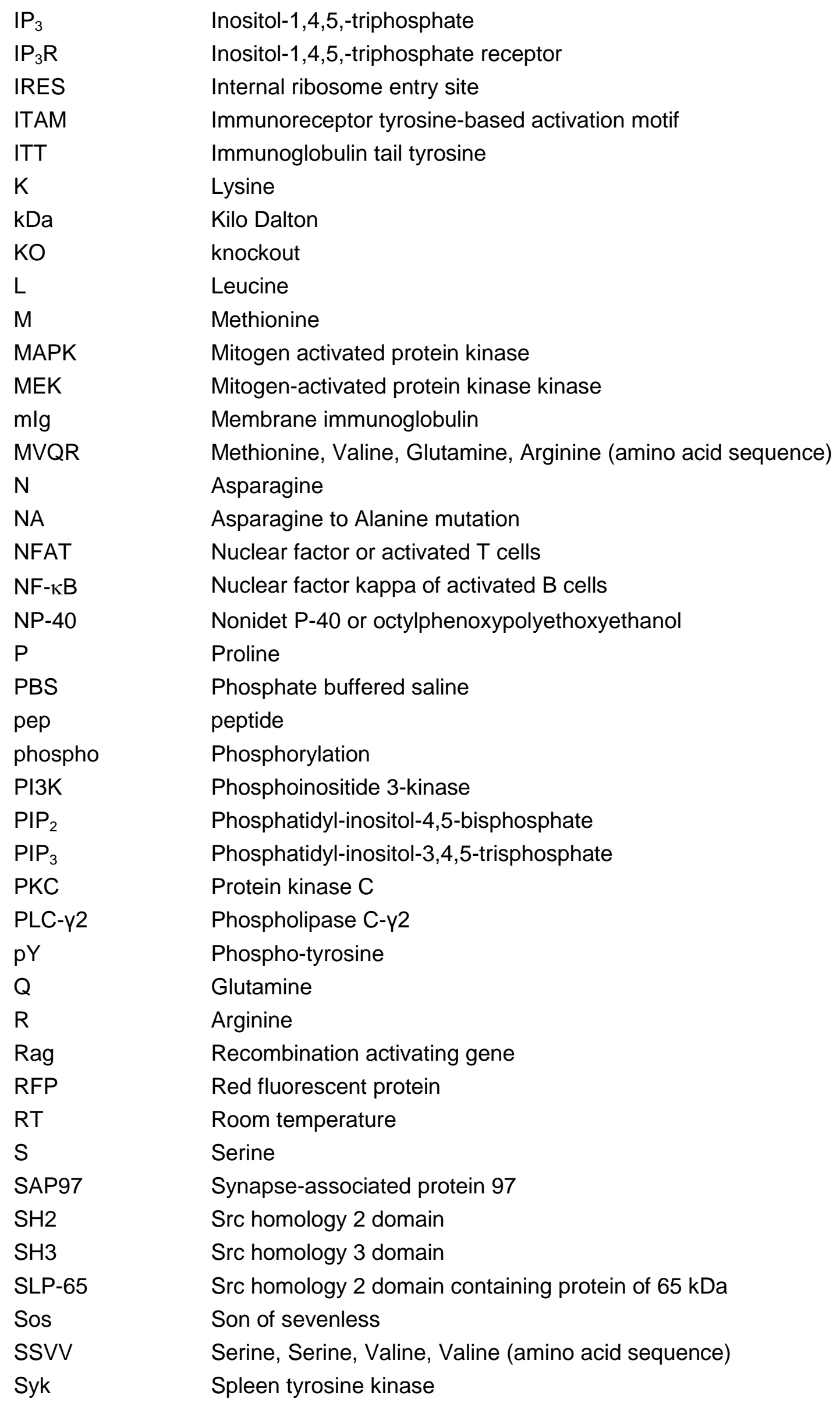




\begin{tabular}{|c|c|}
\hline $\mathrm{T}$ & Threonine or Thymine \\
\hline TM & transmembrane \\
\hline V & Valine \\
\hline $\mathrm{V}(\mathrm{D}) \mathrm{J}$ & $\begin{array}{l}\text { Variable }(V) \text {, Diversity }(D) \text {, Joining }(J) \text { gene segments of the lg heavy } \\
\text { chain or Ig light chain }\end{array}$ \\
\hline W & Tryptophan \\
\hline WT & Wild-type \\
\hline Y & Tyrosine \\
\hline YA & Tyrosine to Alanine mutation \\
\hline $\mathrm{YxN}$ & Tyrosine, $\mathrm{x}$ being any amino acid, Asparagine (amino acid sequence) \\
\hline$\alpha$ & Anti \\
\hline$\lambda L C$ & Lambda light chain \\
\hline$\gamma 2 \mathrm{am}$ & Membrane immunoglobulin G2a \\
\hline$\varepsilon \mathrm{m}$ & Membrane immunoglobulin $\mathrm{E}$ \\
\hline$\varepsilon m s$ & Membrane immunoglobulin E short isoform \\
\hline$\mu \mathrm{m}$ & Membrane immunoglobulin M \\
\hline um & Membrane immunoglobulin $\mathrm{Y}$ \\
\hline
\end{tabular}




\section{List of Figures}

Figure 1: The diverse mlgM-BCR induced signaling pathways converge in the nucleus.

Page

Figure 2: BCR signal amplification by the mlgG-ITT motif alters IgG memory responses.

Figure 3: The ITT is the primary signal amplification module in memory type-BCRs.

Figure 4: The ITT is the sole signaling motif in the mlgE cytoplasmic tail.

Figure 5: The ITT increases the sensitivity of mlgE-BCRs.

Figure 6: The human mlgE-ITT is phosphorylated by the tyrosine kinase Syk.

Figure 7: The human mlgE-ITT is partly functional even in the absence of the adaptor protein, Grb2.

Figure 8: Signal amplification by human mlgE-BCRs depends on the asparagine residue in the ITT motif core.

Figure 9: Mass spectrometric analysis of the phosphorylated mlgE-ITT interactome.

Figure 10: The mlgE-ITT binds to the closely related adaptor proteins Grb2 and Grap.

Figure 11: mlgE-ITT-mediated signal amplification is lost in the absence of Grb2 and Grap.

Figure 12: Reconstitution of the DG75 GRB2/GRAP dKO with the adaptor proteins Grb2

and Grap.

Figure 13: Both Grb2 and Grap contribute to mlgE-ITT-mediated signal amplification.

Figure 14: mlgE- and mlgM-BCR induced ERK activation is dependent on Grb2 and Grap.

Figure 15: The absence of Grb2 and Grap reduces activation of proteins that form the $\mathrm{Ca}^{2+}$ initiation complex.

Figure 16: The $\mathrm{N}$ - and $\mathrm{C}$-terminal SH3 domains of Grb2 have different roles in mlgE- and mlgM-BCR signaling.

Figure 17: The N-terminal SH3 domain of Grap is required for optimal mlgE- and mlgMBCR-mediated signaling.

Figure 18: Grb2 and Grap are recruited to the BCR and/or to co-stimulatory molecules upon BCR activation.

Figure 19: The mlgE-BCR is more dependent on Grb2 and Grap than the mlgM-BCR for signaling in DG75 B cells.

Figure 20: The mlgE-BCR signals despite minimal interaction with $\lg \alpha$ in DG75 B cells.

Figure 21: The human mlgE molecule can pair with $\lg \alpha$.

Figure 22: The human IgE molecule can be expressed on B cells as a functional signaling unit in the absence of the $\lg \alpha-\lg \beta$ heterodimer.

Figure 23: The cytoplasmic tail of human mlgE is sufficient to mediate $\lg \alpha / \lg \beta$-independent expression.

Figure 24: The human mlgE molecule can be expressed on terminally differentiated plasma cells.

Figure 25: Grb2 and Grap are involved in mlgE-ITT-mediated BCR signal amplification and also contribute to canonical ITAM-mediated $\mathrm{Ca}^{2+}$ signaling of mlgE- and mlgM-BCRs.

Figure 26: The human mlgE-ITT can function independently of the $\lg \alpha / \lg \beta$ heterodimer. 


\section{Declaration}

Herewith I declare, that I prepared the Doctoral Thesis titled " The membrane IgE tail imparts unique signaling properties to the B cell antigen receptor " on my own and with no other sources and aids than quoted.

Göttingen, October $25^{\text {th }} 2016$

Kanika Vanshylla 


\section{Curriculum Vitae}

Name: Kanika Vanshylla

Date of birth: 10.01.1990

Place of Birth: New Delhi, India

E-mail: kanika.vanshylla@gmail.com

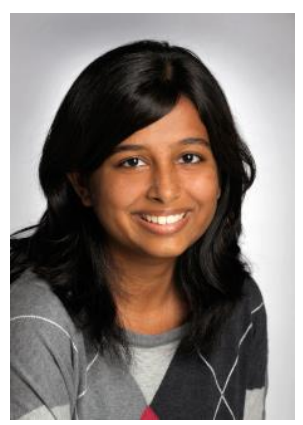

\section{Education}

- 2013-Present Ph.D. position in Immunology

Institute for Cellular and Molecular Immunology, University Medical Center Göttingen, Germany

Project supervisors: Prof. Dr. Jürgen Wienands and Dr. Niklas Engels

- 2011-2013 M.Sc. in Molecular Biology

International Max Planck Research School for Molecular Biology, Göttingen, Germany

Final grade: 1.3 (A-excellent)

\section{- 2008-2011 B.Sc. (Hons) Biochemistry}

Sri Venkateswara College, University of Delhi, India

Final Score: $84 \%$, Rank: $1^{\text {st }}$

- 2008 Higher secondary school

Graduated from Delhi Public School, Vasant Kunj, New Delhi by completing the All India Senior Secondary Examination of the Central Board of Secondary Education. Final Score: 91\%, Final Grade: A

\section{Publications}

- Niklas Engels, Lars M. Koenig, Wiebke Schulze, Daniel Radtke, Kanika Vanshylla, Johannes Lutz, Thomas H. Winkler, Lars Nitschke, Juergen Wienands. The immunoglobulin tail tyrosine motif upgrades memory-type BCRs by incorporating a Grb2-Btk signaling module. Nature Communications. 5:5456 doi: 10.1038/ncomms6456, 2014.

\section{Conference talks}

- Selected talk at the $46^{\text {th }}$ Annual Meeting of the German Society for Immunology, Hamburg, Germany, 27-30 September 2016.

- Selected talk at the International Symposium On B Cells: Immunity And Autoimmunity, Erlangen, Germany, 1-3 October 2015. 


\section{Scholarships and awards}

- 2016

ICGEB scholarship for attending the EMBO Lymphocyte antigen receptor signaling meeting in Pontignano, Siena, Italy, 3-7 September 2016.

IMPRS travel grant for attending the Keystone Symposia: B cells at the intersection of innate and adaptive immunity in Stockholm, Sweden, 29 May- 2 June 2016.

- 2013

PhD bridging fund stipend, Goettingen Graduate School for Neurosciences, Biophysics and Molecular Biosciences (GGNB).

- 2011-2012

Stipend of the Excellence Foundation for the promotion of the Max Planck Society.

- 2011

Gold Medal for $1^{\text {st }}$ rank in B.Sc. (Hons) Biochemistry at the University of Delhi.

\section{Technical laboratory skills}

- Molecular biology, biochemistry, cell biology and immunology techniques including cloning, protein expression and interaction studies, RNA analysis, retroviral transfections, flow cytometry and mammalian cell culture (cell lines and primary cells).

- Laboratory animal science - FELASA B certification.

\section{Additional professional skills}

\section{- Teaching experience}

Supervision of internship students in the lab and conducting classroom tutorials for M.Sc. students of the graduate school.

- Conference organization

Member of the organizing committee of Horizons in Molecular Biology International Symposium held in Göttingen, Germany in 2013 and 2014.

- Languages

English (fluent) and Hindi (native) 